\title{
Asymptotic Behaviour of the Domain of Analyticity of Invariant Curves of the Standard Map
}

\author{
Tere M. Seara and Jordi Villanueva
}

October 18, 1999

Departament de Matemàtica Aplicada I, Universitat Politècnica de Catalunya, Diagonal 647, 08028 Barcelona, Spain.

E-mails: tere@ma1.upc.es, villanueva@ma1.upc.es

\begin{abstract}
In this paper we consider the standard map, and we study the invariant curve obtained by analytical continuation, with respect to the perturbative parameter $\varepsilon$, of the invariant circle of rotation number the golden mean corresponding to the case $\varepsilon=0$. We show that, if we consider the parameterization that conjugates the dynamics of this curve to an irrational rotation, the domain of definition of this conjugation has an asymptotic boundary of analyticity when $\varepsilon \rightarrow 0$ (in the sense of the singular perturbation theory). This boundary is obtained studying the conjugation problem for the so-called semi-standard map.

To prove this result we have used KAM-like methods adapted to the framework of singular perturbation theory, as well as matching techniques to join different pieces of the conjugation, obtained in different parts of its domain of analyticity.
\end{abstract}




\section{Contents}

1 Introduction $\quad 3$

2 The Newton method 6

2.1 Reducibility of invariant curves . . . . . . . . . . . . . . 7

2.2 Formulation of the iterative method . . . . . . . . . . . . 10

2.3 Convergence of the iterative method and uniqueness of solution . . . . . . 12

2.4 Application to the standard map . . . . . . . . . . . . 13

3 The inner variables $\quad 14$

3.1 The inner equation . . . . . . . . . . . . . . . 15

3.2 The Newton method in the inner variables . . . . . . . . . . 16

4 The modified Newton method $\quad 18$

4.1 Formulation of the modified iterative method . . . . . . . . . . . . . 19

4.2 Analysis of the errors in the modified iterative method . . . . . . . . . 22

5 The standard map as a singular perturbation of the semi-standard map 24

5.1 The variational equation of the inner solution . . . . . . . . . 25

5.2 Convergence of the modified Newton method . . . . . . . . . . . 27

5.3 Proof of Theorem 1: complex matching . . . . . . . . . . . . 30

A Proof of the convergence theorems

A.1 Some basic properties of the norm . . . . . . . . . . . . . . 32

A.2 Proof of Theorem 2 and Proposition $1 \ldots \ldots . \ldots . \ldots 33$

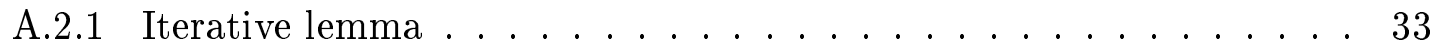

A.2.2 Proof of the convergence . . . . . . . . . . . . 36

A.2.3 Proof of Proposition $1 \ldots \ldots . \ldots . \ldots . \ldots 37$

A.3 Proof of Theorem $3 \ldots \ldots \ldots$. . . . . . . . . . . . . 39

A.3.1 Iterative lemma . . . . . . . . . . . . . . . 39

A.3.2 Proof of the convergence . . . . . . . . . . . . . 45

A.4 Some sharp estimates on the small divisors for the golden mean . . . . . . 47

$\begin{array}{ll}\text { Acknowledgements } & \mathbf{5 0}\end{array}$

$\begin{array}{ll}\text { References } & \mathbf{5 0}\end{array}$ 


\section{Introduction}

We consider the following family of exact symplectic diffeomorphisms of the cylinder to itself,

$$
F:(q, p) \in \mathbb{S}^{1} \times \mathbb{R} \mapsto\left(q+p+V^{\prime}(q), p+V^{\prime}(q)\right) \in \mathbb{S}^{1} \times \mathbb{R},
$$

where $\mathbb{S}^{1}=\mathbb{R} / 2 \pi \mathbb{Z}$ and $V(q)$ is an analytic function, $2 \pi$-periodic in $q$. We will refer to these maps as "standard-like maps". In this context, it is usual to consider the case where $V(q)$ is an even trigonometric polynomial and "small". This smallness can be expressed, for instance, saying that $V \equiv \mathcal{O}(\varepsilon)$, where $\varepsilon$ is a small parameter. In particular, when $V(q)=-\varepsilon \cos q$ we obtain the so-called "standard map" ([4]),

$$
(q, p) \mapsto(q+p+\varepsilon \sin q, p+\varepsilon \sin q) .
$$

In spite of its simple formulation, the standard map displays a very complex behaviour. For this reason, it has become a classical model to study several non-integrability phenomena, such as the creation of hyperbolic objects due to the breakdown of resonant invariant curves, the splitting of separatrices and the chaotic behaviour associated to it, the existence and breakdown of irrational invariant curves, etcetera. We can refer to the extense literature on the topic (see, for instance, [1], [8] and [11]).

If we take $V \equiv 0$ in (1), we obtain an integrable twist map:

$$
(q, p) \in \mathbb{S}^{1} \times \mathbb{R} \mapsto(q+p, p) \in \mathbb{S}^{1} \times \mathbb{R}
$$

For any $p_{0} \in \mathbb{R}$, we have that $\mathbb{S}^{1} \times\left\{p_{0}\right\}$ is an invariant circle of $(3)$, and that the dynamics on the variable $q$ is a rotation of angle $\omega \equiv p_{0}$. If we consider the perturbed map (1), it is natural to ask for which irrational values of the rotation number $\frac{\omega}{2 \pi}$ there exists an invariant curve, close to the unperturbed one, with the same quasi-periodic dynamics. This problem has been considered by several authors, mainly for the case of the standard map, and it is one of the most classical examples of a small divisors problem. To ensure the persistence of the invariant curve the rotational number has to be irrational enough to control the small divisors involved. For instance, if the rotation number is Diophantine, that is,

$$
\left|\frac{\omega}{2 \pi}-\frac{n}{m}\right| \geq \frac{c_{\omega}}{|m|^{\sigma}}, \quad n, m \in \mathbb{Z}, m \neq 0,
$$

for certain $c_{\omega}>0$ and $\sigma \geq 2, \operatorname{KAM}$ theory ([12]) ensures that, if $V(q)$ is small enough, then there exists an invariant analytic curve close to the unperturbed one, having the same quasi-periodic dynamics up to a smooth change of variables. This curve can be found looking for a parameterization $(q, p)=\left(u_{1}(\theta), u_{2}(\theta)\right)$, with $\theta \in \mathbb{S}^{1}$, such that the dynamics on the variable $\theta$ corresponds to the desired irrational rotation, that is, $F\left(u_{1}(\theta), u_{2}(\theta)\right)=$ $\left(u_{1}(\theta+\omega), u_{2}(\theta+\omega)\right)$. Now, using $(1)$, we have that $u_{2}(\theta)=u_{1}(\theta+\omega)-u_{1}(\theta)-V^{\prime}\left(u_{1}(\theta)\right)$, and this system of functional equations can be reduced to the following second order difference equation for $u(\theta) \equiv u_{1}(\theta)$ :

$$
u(\theta+\omega)-2 u(\theta)+u(\theta-\omega)=V^{\prime}(u(\theta)),
$$

usually called Lagrangian formulation of this conjugation problem. Moreover, if we want this curve to be an analytic continuation of the unperturbed one, we have to look for $u(\theta)=\theta+\ell(\theta)$, with $\ell(\theta) 2 \pi$-periodic in $\theta$. We will choose $\ell(\theta)$ with zero average, for instance, in order to avoid the indetermination of the origin for $\theta$. 
In the Diophantine case (4), it is known that, if $V \equiv \mathcal{O}(\varepsilon), \ell(\theta)$ is of the same order for moderate big values of $|\operatorname{Im}(\theta)|$. The aim of this paper is to study the existence and the analytic properties of $\ell(\theta)$ when $|\operatorname{Im}(\theta)|$ grows. In order to present concrete results, and for a sake of simplicity, we are going to consider the case of the standard map and we will work with the invariant curve with rotation number $\gamma$, where $\gamma=\frac{\sqrt{5}-1}{2}$ is the golden mean. Let us introduce the main ideas of our approach.

There are several works in the literature (see, for instance, [6], [7], [10], [3], [9], [2] and [5]) devoted to finding the critical value of the perturbation parameter $\varepsilon$ corresponding to the breakdown of this $\gamma$-invariant curve, since there is a strong evidence that it is the most robust invariant circle of the unperturbed case. Nevertheless, the goal of this paper is to find an asymptotic estimate, when $\varepsilon \rightarrow 0$, for the width of the strip of analyticity of the function $\ell(\theta)$.

A direct application of KAM theory to this context only gives the existence of $\ell(\theta)$, if $\varepsilon$ is small enough, for complex values of $\theta$ with $|\operatorname{Im}(\theta)| \leq C$, where $C>0$ is independent on $\varepsilon$. A more careful analysis, also using KAM-like methods, allows us to improve this estimate in theorem 2 and corollary 1 obtaining that the curve is analytic for $|\operatorname{Im}(\theta)| \leq$ $\log (A / \varepsilon)$, where $A$ is independent on $\varepsilon$ (this result also follows from [9]). Nevertheless, with the direct KAM approach we are far to obtain which is the "optimal" value for $A$ in this expression. This is because KAM methods are based on an iterative process which converges to the solution of equation (5) quadratically (Newton-like method), and therefore, we only can expect convergence in a domain where the solution is a small perturbation of the initial approximation $\ell^{(0)}(\theta)=0$ (which is the solution when $V \equiv 0$ ). In the case of the standard map, if we look for the function $\ell(\theta)$ as a power series with respect to $\varepsilon$, it is straightforward to check that

$$
\ell(\theta)=\frac{\varepsilon \sin \theta}{2(\cos (2 \pi \gamma)-1)}+\mathcal{O}\left(\varepsilon^{2}\right) .
$$

Then, the solution is not perturbative when $|\operatorname{Im}(\theta)|=\log (1 / \varepsilon)+\mathcal{O}(1)$. In order to study the behaviour of $\ell(\theta)$ for $\operatorname{Im}(\theta) \approx-\log (1 / \varepsilon)$, it is natural to perform the change of variables

$$
\theta=-i \log \left(\frac{1}{\varepsilon}\right)+\pi+\tau, \quad l(\tau)=\ell(\theta), \quad v(\tau)=\tau+l(\tau),
$$

where $i=\sqrt{-1}$, and hence,

$$
u(\theta)=\theta+l\left(i \log \left(\frac{1}{\varepsilon}\right)-\pi+\theta\right)=-i \log \left(\frac{1}{\varepsilon}\right)+\pi+v(\tau) .
$$

With this new variable, the boundary of the domain where we will assure convergence of the Newton method (see Section 2.4), corresponds to values of $\tau$ with $\operatorname{Im}(\tau) \equiv \mathcal{O}(1)$. After this $\varepsilon$-dependent change, equation (5) reads

$$
v(\tau+2 \pi \gamma)-2 v(\tau)+v(\tau-2 \pi \gamma)=\frac{i}{2} \mathrm{e}^{i v(\tau)}-\varepsilon^{2} \frac{i}{2} \mathrm{e}^{-i v(\tau)}
$$

Now, if we put $\varepsilon=0$ in (8), we obtain a new unperturbed problem, usually called inner equation in the context of singular perturbation theory. In our case, the inner equation corresponds to the Lagrangian equation (5) for the $\gamma$-invariant curve of the so-called semi-standard map,

$$
(q, p) \mapsto\left(q+p+\frac{i}{2} \mathrm{e}^{i q}, p+\frac{i}{2} \mathrm{e}^{i q}\right)
$$


which is obtained from (1) taking $V(q)=\frac{1}{2} \mathrm{e}^{i q}$. This map was introduced in [7], and it can be seen as a limit for the standard map in the negative half plane for $\operatorname{Im}(\tau)$. Actually, we expect the lower boundary of the domain of analyticity with respect to $\tau$ of the invariant curve of the semi-standard map, to have similar behaviour than the one of the standard map, at least for small values of $\varepsilon$. Assuming that both boundaries have this close behaviour, the semi-standard map has the advantage that the periodic solutions of its Lagrangian equation can be found by a power series expansion, by means of the change $x=\mathrm{e}^{i \tau}$. In Lemma 1, we will see that this power series has a finite radius of convergence $R_{0}>0$. Then, it is natural to expect $R_{0}$ to be the asymptotic value for $A$.

To establish this result, we will prove, in Theorem 3 and Corollary 3 , that the solution of (8) can be obtained, as a perturbation of the inner solution, in a complex domain for the variable $\tau$ of the form $-\log (R(\varepsilon)) \leq \operatorname{Im}(\tau) \leq-\log (B)$, where $\lim _{\varepsilon \rightarrow 0} R(\varepsilon)=R_{0}$, and we will take the constant $B>0$ small enough in order to overlap this complex domain with the domain of analyticity of the invariant curve $u(\theta)=\theta+\ell(\theta)$, obtained by means of the standard KAM approach. Going back to the original variable $\theta$, we prove (using matching techniques) that the solution obtained is the analytic continuation of $u(\theta)$ until $\operatorname{Im}(\theta) \geq-\log (R(\varepsilon) / \varepsilon)$, and so, $-\log \left(R_{0} / \varepsilon\right)$ is the asymptotic lower boundary for its domain of analyticity. To prove this result, we will be forced to modify the Newton method in order to obtain a suitable formulation to work close to the boundary of the strip of analyticity. Of course, by the symmetry of the standard map, we have an analogous result for the upper half plane, giving rise to the following theorem.

Theorem 1 (a) The semi-standard map (9) has an invariant analytic curve of degree one, whose dynamics is analytically conjugated to a rotation of angle $\omega=2 \pi \gamma$. If we denote this conjugation by $\left(v_{1}^{(0)}(\tau), v_{2}^{(0)}(\tau)\right)$, where $v_{1}^{(0)}(\tau)-\tau$ and $v_{2}^{(0)}(\tau)$ are $2 \pi$ periodic analytic functions, with $v_{1}^{(0)}(\tau)-\tau$ having zero average, then, there exists $0<R_{0}<+\infty$, such that the Fourier expansions of $v_{1}^{(0)}(\tau)-\tau$ and $v_{2}^{(0)}(\tau)$ are convergent only if $\operatorname{Im}(\tau) \geq-\log R_{0}$.

(b) The standard map (2), for $\varepsilon$ small enough, has an invariant analytic curve of degree one, whose dynamics is analytically conjugated to a rotation of angle $\omega=2 \pi \gamma$, and which is an analytic continuation, with respect to $\varepsilon$, of the invariant circle $\mathbb{S}^{1} \times\{\omega\}$ corresponding to the case $\varepsilon=0$ (3). If we denote this conjugation by $\left(u_{1}(\theta), u_{2}(\theta)\right)$, where $u_{1}(\theta)-\theta$ and $u_{2}(\theta)$ are $2 \pi$-periodic analytic functions, with $u_{1}(\theta)-\theta$ having zero average, then, we have that they are analytic (at least) in the complex domain for $\theta$ given by

$$
|\operatorname{Im}(\theta)| \leq \log (R(\varepsilon) / \varepsilon),
$$

where $R(\varepsilon)$ behaves, as function of $\varepsilon$, as $R(\varepsilon)=R_{0}-\mathcal{O}\left(\varepsilon^{1 / 4-\alpha}\right)$, for any $\alpha>0$.

(c) When we approach to the lower border of the domain of analyticity, we have that the $\gamma$-invariant curve of the standard map is a small perturbation of the one of the semi-standard map, in the following sense:

There exists a constant $0<D<R_{0}$ with $R_{0}-D$ small enough, such that

$$
\lim _{\varepsilon \rightarrow 0}\left|\left(u_{1}(\theta)-\theta\right)-\left(v_{1}^{(0)}(\tau)-\tau\right)\right|=0, \quad \lim _{\varepsilon \rightarrow 0}\left|u_{2}(\theta)-v_{2}^{(0)}(\tau)\right|=0,
$$

if

$$
-\log (R(\varepsilon) / \varepsilon) \leq \operatorname{Im}(\theta) \leq-\log (D / \varepsilon)
$$


and we take $\tau=\theta+i \log (1 / \varepsilon)-\pi$.

Remark 1 In view of the results displayed in Theorem 1, we can say, roughly speaking, that $\operatorname{Im}(\theta)=-\log \left(R_{0} / \varepsilon\right)$ is, asymptotically, "the natural" (lower) boundary of analyticity of $\left(u_{1}(\theta), u_{2}(\theta)\right)$.

Remark 2 Of course, we have an analogous result than (c) in the upper half plane, but we have to re-formulate (a) in terms of the following "alternative" definition of the semistandard map:

$$
(q, p) \mapsto\left(q+p-\frac{i}{2} \mathrm{e}^{-i q}, p-\frac{i}{2} \mathrm{e}^{-i q}\right)
$$

Remark 3 In spite of that, to present these results we have focused in the invariant curve of the standard map with rotation number the golden mean, the methods used in this paper can be applied to any invariant curve having Diophantine rotation number (4). Moreover, we can also deal with the general case $(1)$, taking $V(q)=\varepsilon P(q)$, with $P(q)$ a trigonometric polynomial.

This paper is organized as follows. In Section 2 we formulate the Newton method used to construct a sequence of functions which converge to the solution of equation (5). This result is summarized in Theorem 2 and its application to the standard map is given in Corollary 1. Section 3 is devoted to the study of the domain of analyticity of the inner solution obtained after the change of variables (6) and (7). In Section 4, we construct the modified Newton method which allows us, in Section 5, to obtain the invariant curve near the inner domain. This result is stated in Theorem 3, and a more quantitative version of the domain of convergence is given in Corollary 3. Finally, the proof of Theorem 1 is also given in Section 5. In Appendix A, we give the technical details of the proofs of several results appearing along the paper.

\section{The Newton method}

In this section we will discuss the formulation of an iterative scheme, based in the standard Newton method, to compute general invariant curves of (1). To do that, we allow any Diophantine $\omega(4)$ and any periodic and entire $V(q)$ (not necessarily small, in principle), and we will study the requirements to ensure convergence in this general context.

First of all, we consider the following linear operators corresponding to first and second order differences with respect to $\omega$ :

$$
\mathcal{L}_{\omega} \xi(\theta)=\xi(\theta+\omega)-\xi(\theta)
$$

and

$$
\mathcal{L}_{\omega}^{2} \xi(\theta)=\xi(\theta+\omega)-2 \xi(\theta)+\xi(\theta-\omega)
$$

We have the following elemental properties:

$$
\mathcal{L}_{\omega}^{2} \xi(\theta+\omega)=\left(\mathcal{L}_{\omega}\right)^{2} \xi(\theta), \quad \mathcal{L}_{\omega}(\xi(\theta) \eta(\theta))=\left(\mathcal{L}_{\omega} \xi(\theta)\right) \eta(\theta+\omega)+\xi(\theta) \mathcal{L}_{\omega} \eta(\theta) .
$$

With this notation, (5) becomes

$$
\mathcal{L}_{\omega}^{2} u(\theta)=V^{\prime}(u(\theta))
$$


Now, let us suppose that we know $u(\theta)=\theta+\ell(\theta)$ an approximate solution of this equation, with error:

$$
e(\theta)=\mathcal{L}_{\omega}^{2} u(\theta)-V^{\prime}(u(\theta)) .
$$

If we want to improve this approximation by means of a Newton method (formulated in an infinite-dimensional space), the correction $\xi(\theta)$ has to verify

$$
\mathcal{L}_{\omega}^{2} \xi(\theta)=V^{\prime \prime}(u(\theta)) \xi(\theta)-e(\theta) .
$$

This is a linear difference equation of second order, with periodic dependence in $\theta$. Due to this periodic dependence, there are no direct methods to solve this equation. Then, it is natural to look for a linear change of variables, $2 \pi$-periodic in $\theta$, that reduces the homogeneous part of (12) to constant coefficients, that is, to use a Floquet-like method for linear difference equations.

\subsection{Reducibility of invariant curves}

To discuss the resolution of equation (12), let us begin by giving some heuristic ideas about the reducibility to constant coefficients of the homogeneous part of such equation. We point that the final method obtained to solve (12) is analogous to the one formulated in [9], where the threshold for the maximum value of $\varepsilon$ for which the $\gamma$-invariant curve of the standard map exists, is studied using computer assisted proofs.

Let us start assuming that $u(\theta)$ is a true solution of (5), and let us consider (12) for an arbitrary $e(\theta)(2 \pi$-periodic in $\theta)$. In this case, we can construct explicitly the periodic change of variables we are looking for. To do that, we put

$$
\zeta_{1}(\theta)=\xi(\theta), \quad \zeta_{2}(\theta)=\mathcal{L}_{\omega} \xi(\theta),
$$

and (12) becomes

$$
\begin{aligned}
& \mathcal{L}_{\omega} \zeta_{1}(\theta)=\zeta_{2}(\theta) \\
& \mathcal{L}_{\omega} \zeta_{2}(\theta)=V^{\prime \prime}(u(\theta+\omega)) \zeta_{1}(\theta)+V^{\prime \prime}(u(\theta+\omega)) \zeta_{2}(\theta)-e(\theta+\omega) .
\end{aligned}
$$

Introducing

$$
A(\theta)=\left(\begin{array}{cc}
0 & 1 \\
V^{\prime \prime}(u(\theta+\omega)) & V^{\prime \prime}(u(\theta+\omega))
\end{array}\right),
$$

we obtain the following linear system of difference equations for $\zeta_{1}(\theta)$ and $\zeta_{2}(\theta)$ :

$$
\mathcal{L}_{\omega}\left(\begin{array}{l}
\zeta_{1}(\theta) \\
\zeta_{2}(\theta)
\end{array}\right)=A(\theta)\left(\begin{array}{l}
\zeta_{1}(\theta) \\
\zeta_{2}(\theta)
\end{array}\right)-\left(\begin{array}{c}
0 \\
e(\theta+\omega)
\end{array}\right) .
$$

Then, what we want to do is to reduce $A(\theta)$ to constant coefficients. First of all, let us note that if $u(\theta)$ is a solution of $(5)$, then $u^{\prime}(\theta)$ is a solution of its variational equation:

$$
\mathcal{L}_{\omega}^{2} y(\theta)=V^{\prime \prime}(u(\theta)) y(\theta)
$$

Assuming that $u^{\prime}(\theta) \neq 0$ for any $\theta \in \mathbb{S}^{1}$ (as $u^{\prime}(\theta)=1+\ell^{\prime}(\theta)$, this holds, for instance, if $\ell(\theta)$ is small enough), and, as in the case of second order differential equations, we can look for another independent solution of (15) taking the form

$$
y(\theta)=c(\theta) u^{\prime}(\theta)
$$


Then, $c(\theta)$ has to verify

$$
\mathcal{L}_{\omega}^{2}\left(c(\theta) u^{\prime}(\theta)\right)=V^{\prime \prime}(u(\theta)) c(\theta) u^{\prime}(\theta)
$$

and hence:

$$
u^{\prime}(\theta+\omega) \mathcal{L}_{\omega} c(\theta)-u^{\prime}(\theta-\omega) \mathcal{L}_{\omega} c(\theta-\omega)=0
$$

Now, if we define

$$
h(\theta)=\mathcal{L}_{\omega} c(\theta),
$$

and we multiply $(17)$ by $u^{\prime}(\theta)$, we obtain the relation:

$$
\mathcal{L}_{\omega}\left(h(\theta) u^{\prime}(\theta) u^{\prime}(\theta+\omega)\right)=0 .
$$

Thus, we can characterize $h(\theta)$ by the following expression

$$
h(\theta) u^{\prime}(\theta) u^{\prime}(\theta+\omega) \equiv \kappa,
$$

with $\kappa$ an arbitrary constant. In order to choose $\kappa$, we note that if we consider the corresponding fundamental matrix of (14),

$$
\Phi(\theta)=\left(\begin{array}{cc}
u^{\prime}(\theta) & y(\theta) \\
\mathcal{L}_{\omega} u^{\prime}(\theta) & \mathcal{L}_{\omega} y(\theta)
\end{array}\right)
$$

it verifies that $\operatorname{det}(\Phi(\theta))=\kappa$. So, it motivates the choice $\kappa=1$. At this point, we introduce

$$
a=\langle h\rangle, \quad h(\theta)=\frac{1}{u^{\prime}(\theta) u^{\prime}(\theta+\omega)},
$$

where

$$
\langle h\rangle=\frac{1}{2 \pi} \int_{0}^{2 \pi} h(\theta) d \theta
$$

is the average of a $2 \pi$-periodic function in $\theta$. Let us remark that, if $h(\theta)$ is a complex analytic function, we can define its average by shifting the path of integration to a suitable $\operatorname{Im}(\theta)$. If $a \neq 0$, it is not possible to compute from (18) a $2 \pi$-periodic solution $c(\theta)$. Of course, this is not a surprise, as the case when $\Phi(\theta)$ is periodic is, clearly, a degenerate one. Thus, if we write $c(\theta)$ as

$$
c(\theta)=\nu(\theta)+\frac{a}{\omega} \theta,
$$

then we can look for $\nu(\theta)$ as a $2 \pi$-periodic solution of

$$
\mathcal{L}_{\omega} \nu(\theta)=\tilde{h}(\theta)
$$

taking $\langle\nu\rangle=0$, for instance, where we define $\widetilde{h}(\theta)$ as

$$
\tilde{h}(\theta)=h(\theta)-\langle h\rangle .
$$

In (21) we find the classical first order difference equation related to the inverse of the operator $\mathcal{L}_{\omega}$. To solve this equation, we expand $h(\theta)$ in Fourier series,

$$
h(\theta)=\sum_{k \in \mathbb{Z}} h_{k} \mathrm{e}^{i k \theta}
$$


and then $\nu(\theta)$ is given by

$$
\nu(\theta)=\mathcal{L}_{\omega}^{-1}(\widetilde{h}(\theta)) \equiv \sum_{k \in \mathbb{Z} \backslash\{0\}} \frac{h_{k}}{\mathrm{e}^{i k \omega}-1} \mathrm{e}^{i k \theta} .
$$

This expression involves small divisors coming from $\omega$, but it is convergent if $h(\theta)$ is analytic and $\frac{\omega}{2 \pi}$ verifies the Diophantine condition (4) (see Lemma 4).

Now, we have constructed a matrix $\Phi(\theta)$ such that $\mathcal{L}_{\omega} \Phi(\theta)=A(\theta) \Phi(\theta)$, and moreover

$$
\Phi(\theta+2 \pi)=\Phi(\theta)\left(\begin{array}{cc}
1 & 2 \pi \frac{a}{\omega} \\
0 & 1
\end{array}\right) .
$$

Then, we proceed to perform the Floquet reduction analogously to the case of periodic systems of ordinary differential equations. We define

$$
B=\left(\begin{array}{cc}
0 & \frac{a}{\omega} \\
0 & 0
\end{array}\right)
$$

and introduce

$$
P(\theta)=\Phi(\theta) \mathrm{e}^{-\theta B}=\left(\begin{array}{cc}
u^{\prime}(\theta) & \nu(\theta) u^{\prime}(\theta) \\
\mathcal{L}_{\omega} u^{\prime}(\theta) & \mathcal{L}_{\omega}\left(\nu(\theta) u^{\prime}(\theta)\right)+a u^{\prime}(\theta+\omega)
\end{array}\right) .
$$

It is clear that $P(\theta)$ is $2 \pi$-periodic in $\theta$, and that $\operatorname{det}(P(\theta))=1$. Now, we perform on (14) the change of variables:

$$
\zeta=P(\theta) \eta
$$

where $\zeta=\left(\zeta_{1}, \zeta_{2}\right)$. To give the transformed system, we use that, by properties $(10)$

$$
\mathcal{L}_{\omega} P(\theta)=\left(\mathcal{L}_{\omega} \Phi(\theta)\right) \mathrm{e}^{-\theta B}+\Phi(\theta+\omega) \mathcal{L}_{\omega} \mathrm{e}^{-\theta B}=A(\theta) P(\theta)-P(\theta+\omega)\left(\begin{array}{cc}
0 & a \\
0 & 0
\end{array}\right),
$$

and then, using again (10) and (26), we obtain for $\eta=\left(\eta_{1}, \eta_{2}\right)$ :

$$
\mathcal{L}_{\omega} \eta(\theta)=\left(\begin{array}{ll}
0 & a \\
0 & 0
\end{array}\right) \eta(\theta)-P(\theta+\omega)^{-1}\left(\begin{array}{c}
0 \\
e(\theta+\omega)
\end{array}\right) .
$$

This is a new system of difference equations of first order, which has the homogeneous part reduced to constant coefficients. For this reason, and with analogy to the case of linear periodic systems of differential equations, we will refer to $P(\theta)$ as the Floquet matrix of the homogeneous part of (14). Moreover, we remark that the non-homogeneous part of (27) can be re-written in the following form:

$$
P^{-1}(\theta)\left(\begin{array}{c}
0 \\
e(\theta)
\end{array}\right)=\left(\begin{array}{c}
-\nu(\theta) \\
1
\end{array}\right) u^{\prime}(\theta) e(\theta) .
$$

where we have taken advantage from the fact that $\operatorname{det}(P(\theta))=1$.

Thus, it is immediate to check that if $a \neq 0$ and $\left\langle u^{\prime} e\right\rangle=0$, we can solve $\eta(\theta)$ from (27), with an indeterminate value for $\left\langle\eta_{1}\right\rangle$. 


\subsection{Formulation of the iterative method}

Now, we want to adapt the methodology explained in Section 2.1 to solve $(12)$ when $u(\theta)$ is not an exact solution of (5). In Section 2.1 we have used that $u(\theta)$ is a true solution of such equation to reduce (12) to (27), which is a system of difference equations with constant coefficients, by means of the (explicit) reducibility of invariant curves. Now, in the case of a quasi-solution, we will be forced to replace reducibility by quasi-reducibility, that is, to reduce equation (12) to a system of difference equations with constant coefficients, except by a "small" non-constant contribution in the homogeneous part. Hence, to "solve" (12) in the context of a quasi-solution, we will only take into account the "reduced" part of the system, and we will add the truncated "non-reduced" remainder to the error due to the Newton method. Then, we will use this scheme to construct a sequence of functions $u^{(n)}(\theta), n \geq 0$, that we will define iteratively, solving equation (5) with an error in the $(n+1)$-step which is "quadratic" with respect to the error in the $n$-step.

Let us start assuming that $u^{(n)}(\theta)=\theta+\ell^{(n)}(\theta)$, with $\left\langle\ell^{(n)}\right\rangle=0$, is an approximate solution of (5) with error $e^{(n)}(\theta)$ as defined in (11). Then, we consider $\nu^{(n)}(\theta)$ as introduced in $(20)$ and $(21)$, and we define $u^{(n+1)}(\theta)=u^{(n)}(\theta)+\xi^{(n)}(\theta)$, with

$$
\xi^{(n)}(\theta)=u^{\prime(n)}(\theta) \eta_{1}^{(n)}(\theta)+\nu^{(n)}(\theta) u^{\prime(n)}(\theta) \eta_{2}^{(n)}(\theta)
$$

where $\eta^{(n)}(\theta)=\left(\eta_{1}^{(n)}(\theta), \eta_{2}^{(n)}(\theta)\right), 2 \pi$-periodic in $\theta$, is obtained by solving the following system of first order difference equations (with constant coefficients):

$$
\mathcal{L}_{\omega} \eta^{(n)}(\theta)=\left(\begin{array}{cc}
0 & a^{(n)} \\
0 & 0
\end{array}\right) \eta^{(n)}(\theta)+\left(\begin{array}{c}
\nu^{(n)}(\theta+\omega) \\
-1
\end{array}\right) u^{\prime(n)}(\theta+\omega) e^{(n)}(\theta+\omega),
$$

where, $a^{(n)}$, defined as in (20), is assumed to be non-zero. We observe that, in order to compute a periodic solution for $\eta_{2}^{(n)}(\theta)$ from $(29)$, we need that $\left\langle u^{\prime(n)} e^{(n)}\right\rangle=0$. Let us check that it holds in the present context. To prove this, we multiply the equation defining $e^{(n)}(\theta)$ (see (11)) by $u^{\prime(n)}(\theta)$. Then, we have:

$$
u^{\prime(n)}(\theta) e^{(n)}(\theta)=\mathcal{L}_{\omega}^{2} \ell^{(n)}(\theta)+\left(\mathcal{L}_{\omega}^{2} \ell^{(n)}(\theta)\right) \ell^{\prime(n)}(\theta)-V^{\prime}\left(u^{(n)}(\theta)\right) u^{\prime(n)}(\theta) .
$$

Thus, as $\mathcal{L}_{\omega}^{2} \ell^{(n)}(\theta)$ and $V^{\prime}\left(u^{(n)}(\theta)\right) u^{\prime(n)}(\theta)=\frac{d}{d \theta} V\left(u^{(n)}(\theta)\right)$ have zero average, we have

$$
\begin{aligned}
\left\langle u^{\prime(n)} e^{(n)}\right\rangle & =\left\langle\left(\mathcal{L}_{\omega}^{2} \ell^{(n)}\right) \ell^{\prime(n)}\right\rangle=\frac{1}{2 \pi} \int_{0}^{2 \pi}\left(\ell^{(n)}(\theta+\omega)-2 \ell^{(n)}(\theta)+\ell^{(n)}(\theta-\omega)\right) \ell^{\prime(n)}(\theta) d \theta \\
& =\frac{1}{2 \pi} \int_{0}^{2 \pi}\left(\ell^{(n)}(\theta+\omega) \ell^{\prime(n)}(\theta)-2 \ell^{(n)}(\theta) \ell^{\prime(n)}(\theta)+\ell^{(n)}(\theta) \ell^{\prime(n)}(\theta+\omega)\right) d \theta \\
& =\frac{1}{2 \pi}\left[\ell^{(n)}(\theta+\omega) \ell^{(n)}(\theta)-\left(\ell^{(n)}(\theta)\right)^{2}\right]_{0}^{2 \pi}=0
\end{aligned}
$$

Since the equation for $\mathcal{L}_{\omega} \eta_{2}^{(n)}(\theta)$ holds for any value of $\left\langle\eta_{2}^{(n)}\right\rangle$, we will choose it so as to have a periodic solution for $\eta_{1}^{(n)}(\theta)$, that is,

$$
\left\langle\eta_{2}^{(n)}\right\rangle=-\frac{1}{a^{(n)}}\left\langle\nu^{(n)} u^{(n)} e^{(n)}\right\rangle .
$$

Moreover, we remark that we also have a free choice for $\left\langle\eta_{1}^{(n)}\right\rangle$. Then, we define it to keep zero average for $\ell^{(n+1)}(\theta)$,

$$
\left\langle\eta_{1}^{(n)}\right\rangle=-\left\langle u^{\prime(n)} \widetilde{\eta}_{1}^{(n)}\right\rangle-\left\langle\nu^{(n)} u^{(n)} \eta_{2}^{(n)}\right\rangle
$$


The important thing in this method is that if we consider $u^{(n+1)}(\theta)$ defined in such a way, then we have that $e^{(n+1)}(\theta)$ is $\mathcal{O}\left(\left(e^{(n)}\right)^{2}\right)$. To prove this, we write the new error as

$$
\begin{aligned}
e^{(n+1)}(\theta) & =\mathcal{L}_{\omega}^{2} u^{(n+1)}(\theta)-V^{\prime}\left(u^{(n+1)}(\theta)\right) \\
& =e^{(n)}(\theta)+V^{\prime}\left(u^{(n)}(\theta)\right)-V^{\prime}\left(u^{(n+1)}(\theta)\right)+\mathcal{L}_{\omega}^{2} \xi^{(n)}(\theta) \\
& \equiv e_{1}^{(n+1)}(\theta)+e_{2}^{(n+1)}(\theta),
\end{aligned}
$$

where

$$
\begin{aligned}
& e_{1}^{(n+1)}(\theta)=\mathcal{L}_{\omega}^{2} \xi^{(n)}(\theta)-V^{\prime \prime}\left(u^{(n)}(\theta)\right) \xi^{(n)}(\theta)+e^{(n)}(\theta) \\
& e_{2}^{(n+1)}(\theta)=V^{\prime}\left(u^{(n)}(\theta)\right)+V^{\prime \prime}\left(u^{(n)}(\theta)\right) \xi^{(n)}(\theta)-V^{\prime}\left(u^{(n)}+\xi^{(n)}(\theta)\right)
\end{aligned}
$$

As it is clear that $e_{2}^{(n+1)}(\theta)$ is $\mathcal{O}\left(\left(\xi^{(n)}\right)^{2}\right.$ ) (and hence $\mathcal{O}\left(\left(e^{(n)}\right)^{2}\right)$ ), then we only have to discuss the smallness of $e_{1}^{(n+1)}(\theta)$. For this purpose, we remark that if we put $\zeta^{(n)}(\theta)=$ $\left(\zeta_{1}^{(n)}(\theta), \zeta_{2}^{(n)}(\theta)\right)$, with

$$
\zeta_{1}^{(n)}(\theta)=\xi^{(n)}(\theta), \quad \zeta_{2}^{(n)}(\theta)=\mathcal{L}_{\omega} \xi^{(n)}(\theta)
$$

then, by (28) we have

$$
\eta^{(n)}(\theta)=\left(P^{(n)}(\theta)\right)^{-1} \zeta^{(n)}(\theta),
$$

where $P^{(n)}(\theta)$ is defined as $P(\theta)$ in $(25)$, just adding the super-script $(n)$ to the formula. As in the case of $P(\theta)$, the matrix $P^{(n)}(\theta)$ is still being $2 \pi$-periodic in $\theta$, and, from the definition $(21)$ of $\nu^{(n)}(\theta)$, we also have that $\operatorname{det}\left(P^{(n)}(\theta)\right)=1$. Nevertheless, as $u^{(n)}(\theta)$ is not an exact solution of (5), then the matrix $\Phi^{(n)}(\theta)$ (given as in (19)) is not an exact solution of the homogeneous part of the system of linear difference equations associated to the Newton method at the $n$-step (see (14)). Consequently, we can not ensure that the linear transformation defined by $P^{(n)}(\theta)$ reduces this homogeneous part to constant coefficients, and so $\zeta^{(n)}(\theta)$ it is not a solution of equation (14) at the $n$-step. In order to obtain the equation verified by $\zeta^{(n)}(\theta)$, it is straightforward to check that

$$
\mathcal{L}_{\omega} \Phi^{(n)}(\theta)=A^{(n)}(\theta) \Phi^{(n)}(\theta)+\left(\begin{array}{cc}
0 & 0 \\
1 & c^{(n)}(\theta+\omega)
\end{array}\right) e^{\prime(n)}(\theta+\omega)
$$

and hence the matrix $P^{(n)}(\theta)$ verifies, instead of $(26)$,

$$
\mathcal{L}_{\omega} P^{(n)}(\theta)=A^{(n)}(\theta) P^{(n)}(\theta)-P^{(n)}(\theta+\omega)\left(\begin{array}{cc}
0 & a^{(n)} \\
0 & 0
\end{array}\right)+\left(\begin{array}{cc}
0 & 0 \\
1 & \nu^{(n)}(\theta+\omega)
\end{array}\right) e^{(n)}(\theta+\omega) .
$$

This implies that

$$
\mathcal{L}_{\omega} \zeta^{(n)}(\theta)=A^{(n)}(\theta) \zeta^{(n)}(\theta)-\left(\begin{array}{c}
0 \\
e^{(n)}(\theta+\omega)
\end{array}\right)+e^{(n)}(\theta+\omega)\left(\begin{array}{cc}
0 & 0 \\
1 & \nu^{(n)}(\theta+\omega)
\end{array}\right) \eta^{(n)}(\theta) .
$$

Then, writing this equality by components we obtain

$$
\begin{aligned}
\mathcal{L}_{\omega} \zeta_{1}^{(n)}(\theta)= & \zeta_{2}^{(n)}(\theta) \\
\mathcal{L}_{\omega} \zeta_{2}^{(n)}(\theta)= & V^{\prime \prime}\left(u^{(n)}(\theta+\omega)\right) \zeta_{1}^{(n)}(\theta)+V^{\prime \prime}\left(u^{(n)}(\theta+\omega)\right) \zeta_{2}^{(n)}(\theta) \\
& -e^{(n)}(\theta+\omega)+e^{\prime(n)}(\theta+\omega)\left(\eta_{1}^{(n)}(\theta)+\nu^{(n)}(\theta+\omega) \eta_{2}^{(n)}(\theta)\right),
\end{aligned}
$$

and, from here, we derive the following expression for $e_{1}^{(n+1)}$ :

$$
e_{1}^{(n+1)}(\theta)=e^{\prime(n)}(\theta)\left(\eta_{1}^{(n)}(\theta-\omega)+\nu^{(n)}(\theta) \eta_{2}^{(n)}(\theta-\omega)\right) .
$$




\subsection{Convergence of the iterative method and uniqueness of so- lution}

We will work with $2 \pi$-periodic analytic functions defined in complex domains of the form

$$
\mathcal{D}\left(\rho_{1}, \rho_{2}\right)=\left\{z \in \mathbb{C}: \rho_{1} \leq \operatorname{Im}(\theta) \leq \rho_{2}\right\}
$$

with $-\infty \leq \rho_{1} \leq \rho_{2} \leq+\infty$. When working with these domains, we will not restrict to the case when $\mathcal{D}\left(\rho_{1}, \rho_{2}\right)$ is symmetric with respect to the real axis. Moreover, we will also allow one of the boundaries to go to infinity in the purely imaginary direction. To control the size of $2 \pi$-periodic analytic functions $f(\theta)$, defined in $(37)$, we will use the following weighted norm:

$$
\|f(\theta)\|_{\rho_{1}, \rho_{2}}=\left|f_{0}\right|+\sum_{k>0}\left|f_{k}\right| \mathrm{e}^{-k \rho_{1}}+\sum_{k<0}\left|f_{k}\right| \mathrm{e}^{-k \rho_{2}},
$$

defined from the Fourier expansion of $f(\theta)$ (see $(23)$ ). In principle, one can think that it is more natural to work with the standard supremum norm in the desired domain. Nevertheless, as we will see in the proofs, to work with this weighted norm simplifies the estimates we will do on the $2 \pi$-periodic functions involved. Of course, $\|\cdot\|_{\rho_{1}, \rho_{2}}$ is an upper

bound for the supremum norm. Besides, this definition keeps some useful properties of the supremum norm, that we display in Lemma 4 of Section A.1.

Now, let us study the convergence of the iterative method described in Section 2.2. It is clear that to ensure this convergence, we need to have an initial approximation, $u^{(0)}(\theta)=$ $\theta+\ell^{(0)}(\theta)$, with error $e^{(0)}(\theta)$ (see $(11)$ ), small in some complex domain $\mathcal{D}\left(\rho_{1}^{(0)}, \rho_{2}^{(0)}\right)$. Nevertheless, as we are going to see, the smallness of $\left\|e^{(0)}(\theta)\right\|_{\rho_{1}^{(0)}, \rho_{2}^{(0)}}$ is not the only condition to take into account to control the iterative process. Hence, to formulate rigorously the convergence of the method, we have to consider constants $\mu_{1}^{(0)}, \ldots, \mu_{7}^{(0)}$ such that

$$
\begin{aligned}
\left\|\ell^{(0)}(\theta)\right\|_{\rho_{1}^{(0)}, \rho_{2}^{(0)}} & \leq \mu_{1}^{(0)}, \quad\left\|\ell^{(0)}(\theta)\right\|_{\rho_{1}^{(0)}, \rho_{2}^{(0)}} \leq \mu_{2}^{(0)}, \\
\left\|e^{(0)}(\theta)\right\|_{\rho_{1}^{(0)}, \rho_{2}^{(0)}} & \leq \mu_{3}^{(0)}, \quad\left\|\frac{1}{u^{(0)}(\theta)}\right\|_{\rho_{1}^{(0)}, \rho_{2}^{(0)}} \leq \mu_{4}^{(0)}, \\
0<\mu_{5}^{(0)} \leq\left|a^{(0)}\right| & \leq \mu_{6}^{(0)}, \quad\left\|V^{\prime \prime \prime}(q)\right\|_{\rho_{1}^{(0)}-\mu_{1}^{(0)}-1, \rho_{2}^{(0)}+\mu_{1}^{(0)}+1} \leq \mu_{7}^{(0)},
\end{aligned}
$$

where $a^{(0)}$ is the value of $a$ defined in (20) corresponding to $u^{(0)}(\theta)$.

Then we can formulate the following result for the convergence of the iterative method. The proof is given in Section A.2.

Theorem 2 Let be $\omega \in \mathbb{R}$ such that $\frac{\omega}{2 \pi}$ is a Diophantine number (4), for certain $\sigma \geq 2$ and $c_{\omega}>0$, and let be $V(q)$ an entire function, $2 \pi$-periodic in $q$. Let $\ell^{(0)}(\theta)$ be an analytic function defined in $\mathcal{D}\left(\rho_{1}^{(0)}, \rho_{2}^{(0)}\right)$, for certain $-\infty \leq \rho_{1}^{(0)}<\rho_{2}^{(0)} \leq+\infty, 2 \pi$-periodic in $\theta$, and with $\left\langle\ell^{(0)}\right\rangle=0$. We consider the constants $\mu_{j}^{(0)}, j=1, \ldots, 7$, introduced in (39), and we assume that $\mu_{3}^{(0)} \leq 1$. Then, there exists a constant $c \geq 1$, depending on $\mu_{1}^{(0)}, \mu_{2}^{(0)}, \mu_{4}^{(0)}, \mu_{5}^{(0)}, \mu_{6}^{(0)}$ and $\mu_{7}^{(0)}$, such that given any $\delta^{(0)}>0$ verifying

$$
\delta^{(0)}<\min \left\{1, \frac{\rho_{2}^{(0)}-\rho_{1}^{(0)}}{24}\right\}
$$


and provided that $\mu_{3}^{(0)}$ is small enough such that

$$
2 c \mu_{3}^{(0)}\left(\frac{2}{\rho_{2}^{(0)}-\rho_{1}^{(0)}}\right)^{2 \sigma-1} \leq \min \left\{\frac{1}{2} \mu_{5}^{(0)}, \mu_{6}^{(0)}\right\}, \quad \Delta \equiv c\left(\frac{16}{\left(\delta^{(0)}\right)^{4}}\right)^{\sigma-1} \mu_{3}^{(0)}<1
$$

we can ensure that the sequence $u^{(n)}(\theta)$ defined in Section 2.2, beginning with $u^{(0)}(\theta)=$ $\theta+\ell^{(0)}(\theta)$, converges to an analytic solution of $(5), u(\theta)=\theta+\ell(\theta)$, with $\ell(\theta) 2 \pi$-periodic in $\theta$, defined in $\mathcal{D}\left(\rho_{1}^{(0)}+6 \delta^{(0)}, \rho_{2}^{(0)}-6 \delta^{(0)}\right)$ and with $\langle\ell\rangle=0$.

Moreover, in this domain we have for $\ell(\theta)$ the bounds (39), now with $\mu_{1}=\mu_{1}^{(0)}+$ $2 c \mu_{3}^{(0)} /\left(\delta^{(0)}\right)^{2(\sigma-1)}, \mu_{2}=\mu_{2}^{(0)}+2 c \mu_{3}^{(0)} /\left(\delta^{(0)}\right)^{2 \sigma-1}, \mu_{4}=\mathrm{e} \mu_{4}^{(0)}, \mu_{5}=\mu_{5}^{(0)} / 2$ and $\mu_{6}=2 \mu_{6}^{(0)}$, where $\mu_{1} \leq \mu_{1}^{(0)}+1$ and $\mu_{2} \leq \mu_{2}^{(0)}+1$.

On the other hand $\ell(\theta)$ is a small perturbation of $\ell^{(0)}(\theta)$ in the sense that

$$
\begin{gathered}
\left\|\ell(\theta)-\ell^{(0)}(\theta)\right\|_{\rho_{1}^{(0)}+6 \delta^{(0)}, \rho_{2}^{(0)}-6 \delta^{(0)}} \leq 2 c \mu_{3}^{(0)} /\left(\delta^{(0)}\right)^{2(\sigma-1)} \\
\left\|\ell^{\prime}(\theta)-\ell^{(0)}(\theta)\right\|_{\rho_{1}^{(0)}+6 \delta^{(0)}, \rho_{2}^{(0)}-6 \delta^{(0)}} \leq 2 c \mu_{3}^{(0)} /\left(\delta^{(0)}\right)^{2 \sigma-1} .
\end{gathered}
$$

Remark 4 This result give us the functions $\left(u_{1}(\theta), u_{2}(\theta)\right)$, with $u_{1}(\theta)=u(\theta)$ and $u_{2}(\theta)=$ $u_{1}(\theta+\omega)-u_{1}(\theta)-V^{\prime}\left(u_{1}(\theta)\right)$, which are a parameterization of an invariant curve of (1), with domain of analyticity at least $\mathcal{D}\left(\rho_{1}^{(0)}+6 \delta^{(0)}, \rho_{2}^{(0)}-6 \delta^{(0)}\right)$. Moreover, with this parameterization we have that the dynamics on the curve is conjugated to a rotation of angle $\omega$ in the variable $\theta$.

Nevertheless, Theorem 2 only gives the existence of a true solution of equation (5), that is close to the first approximation $u^{(0)}(\theta)$, but does not guarantee, in principle, that this is the only one. However, we can adapt the proof of Theorem 2 to obtain the following result about uniqueness. See also Section A.2 for the proof.

Proposition 1 With the same hypotheses of Theorem 2, let us assume that $\bar{u}(\theta)=\theta+$ $\bar{\ell}(\theta), 2 \pi$-periodic in $\theta$, analytic in $\mathcal{D}\left(\bar{\rho}_{1}, \bar{\rho}_{2}\right)$, where $\bar{\rho}_{1}>\rho_{1}^{(0)}+6 \delta^{(0)}$, and $\bar{\rho}_{2}<\rho_{2}^{(0)}-6 \delta^{(0)}$, and with $\langle\bar{\ell}\rangle=0$, is another solution of (5). Then, if we have that $u(\theta)$ and $\bar{u}(\theta)$ are close enough,

$$
\|\bar{\ell}(\theta)\|_{\bar{\rho}_{1}, \bar{\rho}_{2}} \leq 1+\mu_{1}^{(0)}, \quad\|\bar{\ell}(\theta)-\ell(\theta)\|_{\bar{\rho}_{1}, \bar{\rho}_{2}} \leq \bar{\Delta}
$$

where

$$
\bar{\Delta}=\frac{2\left(\bar{\rho}_{2}-\bar{\rho}_{1}\right)^{2(\sigma-1)}}{c \mu_{7}^{(0)} 2^{6(\sigma-1)}}
$$

then we can assure that $u \equiv \bar{u}$.

\subsection{Application to the standard map}

Even though Theorem 2 can be applied to the general map (1), and for an arbitrary Diophantine $\frac{\omega}{2 \pi}$, now we want to give more concrete results by considering the standard map, with $V(q)=-\varepsilon \cos q$, and more concretely, the invariant curve with rotation number $\gamma$. Thus, we have that $\omega=2 \pi \gamma$ verifies (4) with $\sigma=2$, for certain constant $c_{2 \pi \gamma}>0$, that, in order to simplify the notations, we will denote by $c_{\gamma}$. In this case, we start by taking $u^{(0)}(\theta)=\theta$ as first approximation, which is the solution of (5) for $\varepsilon=0$. With this choice 
we have, independently on the domain, that $\mu_{1}^{(0)}=\mu_{2}^{(0)}=0, \mu_{4}^{(0)}=1$ and $a^{(0)}=1$. On the other hand, as $e^{(0)}(\theta)=-\varepsilon \sin \theta$, it is clear that $\mu_{3}^{(0)}$ depends on the initial domain. In order to choose this domain, let us point that as we are working with real analytic functions, it is natural to consider a symmetric domain with respect to the real axis. If we start with $\mathcal{D}\left(-\rho^{(0)}, \rho^{(0)}\right)$, then we have that $\left\|e^{(0)}(\theta)\right\|_{-\rho^{(0)}, \rho^{(0)}}=\varepsilon \mathrm{e}^{\rho^{(0)}} \equiv \mu_{3}^{(0)}$. Our interest is to look for the largest $\rho^{(0)}$ for which Theorem 2 can be applied, for $\varepsilon$ small enough. This requires $\mu_{3}^{(0)}$ to be small. Hence, the "optimal" selection for $\rho^{(0)}$ seems to be of the form $\rho^{(0)}=\log \left(A^{(0)} / \varepsilon\right)$, where we will need $A^{(0)}>0$ to be "small".

Corollary 1 We consider $\omega=2 \pi \gamma$, which is of Diophantine type (4), with $\sigma=2$ and $c_{\omega} \equiv c_{\gamma}$. Let us take $0<A^{(0)}<\frac{1}{16 c}$, where $c \geq 1$ is the constant provided by Theorem 2 associated to the values $\mu_{1}^{(0)}=\mu_{2}^{(0)}=0, \mu_{4}^{(0)}=\mu_{5}^{(0)}=\mu_{6}^{(0)}=1$, and $\mu_{7}^{(0)}=\mathrm{e} / 2$.

Then, if $\varepsilon \leq \min \left\{\frac{A^{(0)}}{\mathrm{e}^{12}}, \frac{A^{(0)}}{\mathrm{e}^{\left(4 c A^{(0)}\right)^{1 / 3}}}\right\}$, the Lagrangian equation (5) associated to the standard map, has a solution $u(\theta)=\theta+\ell(\theta)$, where $\ell(\theta)$ is $2 \pi$-periodic in $\theta$, with $\langle\ell\rangle=0$ and analytic at least in the complex strip $\mathcal{D}(-\log (A / \varepsilon), \log (A / \varepsilon))$, with $A=A^{(0)} / \mathrm{e}^{6 \delta^{(0)}}$, being $\delta^{(0)}$ any value verifying $\left(16 c A^{(0)}\right)^{1 / 4}<\delta^{(0)}<1$.

Moreover, we have the estimate $\|\ell(\theta)\|_{-\log (A / \varepsilon), \log (A / \varepsilon)} \leq 2 c A^{(0)} /\left(\delta^{(0)}\right)^{2} \leq \sqrt{c A^{(0)} / 3}$.

Proof: The proof of this result is straightforward. We only have to check the statement of Theorem 2, with $V(q)=-\varepsilon \cos q$, by taking $\rho_{2}^{(0)}=-\rho_{1}^{(0)}=\log \left(A^{(0)} / \varepsilon\right)$. So, we have that $\mu_{3}^{(0)}=A^{(0)}$. In addition, condition $\varepsilon \leq A^{(0)} / 12$ guarantee that $\left(\rho_{2}^{(0)}-\rho_{1}^{(0)}\right) / 24 \geq 1$ in (40), and in the same way the remaining conditions (41) are easily verified, and the result follows.

\section{The inner variables}

Corollary 1 ensures the existence of the solution $u(\theta)$ of equation (5) for complex $\theta$, with $|\operatorname{Im}(\theta)| \leq \log (A / \varepsilon)$, for some constant $A>0$. We do not claim that the constant $A$ given by Corollary 1 is optimal. On one hand, the estimates used in the proof can be improved. But even though these estimates were as optimal as possible, we could not ensure that, with the previous approach, we can reach the "optimal" value of $A$. The reason is clear: the Newton method needs the initial error $\mu_{3}^{(0)}$ to be small, or what is the same, the solution $u(\theta)$ to be a small perturbation of the first approximation $u^{(0)}(\theta)=\theta$. Expanding $\ell(\theta)$ in Taylor series with respect to $\varepsilon$, one can check that

$$
\ell(\theta)=\frac{\varepsilon \sin \theta}{2(\cos (2 \pi \gamma)-1)}+\frac{\varepsilon^{2} \sin 2 \theta}{8(\cos (2 \pi \gamma)-1)(\cos (4 \pi \gamma)-1)}+\mathcal{O}\left(\varepsilon^{3}\right)
$$

It is clear that if $|\operatorname{Im}(\theta)|=\log (1 / \varepsilon)+\mathcal{O}(1)$, both terms displayed contribute to the final solution with order 1 with respect to $\varepsilon$. A more careful analysis gives that for $|\operatorname{Im}(\theta)|=\log (1 / \varepsilon)+\mathcal{O}(1)$, all the terms in this series are of order 1 , and hence, the perturbative analysis with respect to $\varepsilon$ fails. This phenomenon suggests us to perform the change of variables given by (6) and (7) in order to study the behaviour of $\ell(\theta)$ for $\operatorname{Im}(\theta) \approx-\log (1 / \varepsilon)$. This change of variables transforms equation (5) into equation (8) for $v(\tau)$. Therefore, for values of $\tau$ with $\operatorname{Im}(\tau) \equiv \mathcal{O}(1)$ it is natural to ask if $v(\tau)$ can be 
find as a perturbation of $v^{(0)}(\tau)=\tau+l^{(0)}(\tau)$, with $l^{(0)}(\tau)=m(\tau)$ defined as the periodic solution, with zero average, of the inner equation:

$$
\mathcal{L}_{2 \pi \gamma}^{2} m(\tau)=\frac{i}{2} \mathrm{e}^{i(\tau+m(\tau))}
$$

Before considering this perturbative analysis, in the next section we are going to study the existence and the domain of definition of $m(\tau)$.

\subsection{The inner equation}

First of all, we need to know that equation (44) has a solution $m(\tau)$ for some range of complex values of $\tau$. If $\operatorname{Im}(\tau)$ is big enough, it is natural to look for this solution as a perturbation of $m^{(0)}(\tau)=0$, and this can be done applying Theorem 2 to equation (44), obtaining:

Corollary 2 We consider $\omega=2 \pi \gamma$, which is of Diophantine type (4) with $\sigma=2$ and $c_{\gamma} \equiv c_{\omega}$. Let us take $c \geq 1$ the constant provided by Theorem 2 associated to the values $\mu_{1}^{(0)}=\mu_{2}^{(0)}=0, \mu_{4}^{(0)}=\mu_{5}^{(0)}=\mu_{6}^{(0)}=1$, and $\mu_{7}^{(0)}=\mathrm{e} / 2$, and let $\rho^{(0)}>\max \{0, \log (8 c)\}$.

Then, equation (44) has a solution $m(\tau), 2 \pi$-periodic in $\tau$, with $\langle m\rangle=0$ and analytic at least in the complex strip $\mathcal{D}\left(\rho^{(0)}+6 \delta^{(0)},+\infty\right)$, where $\delta^{(0)}$ is any number verifying $\left(8 c \mathrm{e}^{-\rho^{(0)}}\right)^{1 / 4}<\delta^{(0)}<1$.

Proof: To prove this result we use again Theorem 2 applied to equation (5), now with $V(q)=\frac{i}{2} \mathrm{e}^{i q}$, and taking $\rho_{1}^{(0)}=\rho^{(0)}$ and $\rho_{2}^{(0)}=+\infty$. Then, $\mu_{3}^{(0)}=\mathrm{e}^{-\rho^{(0)}} / 2$. With this choice we have that $v(\tau)=\tau+m(\tau)$ is the solution we are looking for.

Corollary 2 only guarantees the existence of an analytic solution $m(\tau)$ when the imaginary part of $\tau$ is big enough. But, due to its perturbative character again, this theorem is not useful to know what happens for the values of $\tau$ outside this perturbative range. For instance, with this result we are not able to decide if the solution $m(\tau)$ is entire or if it has some natural lower boundary.

To study this solution with more detail, let us point that, in this case, $m(\tau)$ has only positive Fourier harmonics. To compute the Fourier coefficients of $m(\tau)$, we introduce $x \equiv \mathrm{e}^{i \tau}$, and define $\bar{m}(x) \equiv i m(\tau)$. Then, $\bar{m}(x)$ takes the form

$$
\bar{m}(x)=\sum_{k \geq 1} m_{k} x^{k}
$$

with $m_{k} \in \mathbb{C}$ (as we want $\langle m\rangle=0$, we take $m_{0}=0$ ). Moreover, $\bar{m}(x)$ verifies

$$
\bar{m}(x \Omega)-2 \bar{m}(x)+\bar{m}(x / \Omega)=-\frac{x}{2} \mathrm{e}^{\bar{m}(x)},
$$

where $\Omega=\mathrm{e}^{i \omega}$, with $\omega=2 \pi \gamma$. In Corollary 2 we have proved that $\bar{m}(x)$ has a positive radius of convergence $R_{0}$, for certain $R_{0} \geq \mathrm{e}^{\rho^{(0)}+6 \delta^{(0)}}>0$.

Let us display the recurrence for the coefficients $m_{k}$. For this purpose, we put $F(x)=$ $\mathrm{e}^{\bar{m}(x)}$, and expand

$$
F(x)=\sum_{k \geq 0} F_{k} x^{k},
$$


having $F_{0}=1$ and, using that $F^{\prime}(x)=\bar{m}^{\prime}(x) F(x)$ one obtains

$$
F_{k}=\frac{1}{k} \sum_{j=1}^{k} j m_{j} F_{k-j} .
$$

Thus, it is straightforward to check that

$$
m_{k+1}=\frac{F_{k}}{4 D_{k+1}}
$$

for any $k \geq 0$, where we define $D_{k}=1-\cos (k \omega)=2(\sin (k \omega / 2))^{2}$. By the way, we have that all the coefficients $m_{k}$ are real and positive, for any $k \geq 1$. Using this fact and equation (46), it is not difficult to check that $\bar{m}(x)$ has a finite radius of convergence. (See [5] for a similar result for curves with rotation number of Brjuno type.)

Lemma 1 The solution $\bar{m}(x)$ of equation (46), with $\bar{m}(0)=0$, has a finite radius of convergence $R_{0} \leq 8 / \mathrm{e}$. Moreover, its Taylor expansion converges uniformly up to the border of the disk of convergence, $|x| \leq R_{0}$.

Proof: We take any $0 \leq r<R_{0}$, and we start considering the real part of (46) evaluated at $x=r$. As $\bar{m}(r \Omega)$ and $\bar{m}(r / \Omega)$ are complex conjugated, we have

$$
2(\operatorname{Re}(\bar{m}(r \Omega))-\bar{m}(r))=-\frac{r}{2} \mathrm{e}^{\bar{m}(r)} .
$$

Then, as the coefficients $m_{k}$ are positive, we have that

$$
\max _{|x|=r}|\operatorname{Re}(\bar{m}(x))|=\bar{m}(r) .
$$

From here, we deduce

$$
\bar{m}(r) \geq \frac{r}{8} \mathrm{e}^{\bar{m}(r)}
$$

or in an equivalent way,

$$
r \leq 8 \bar{m}(r) \mathrm{e}^{-\bar{m}(r)} .
$$

Now, if we assume non-bounded radius of convergence, we will have that $\lim _{r \rightarrow \infty} \bar{m}(r)=$ $+\infty$. But if we take this limit at the right-hand-side of the previous expression, we have a contradiction, as $8 \bar{m}(r) \mathrm{e}^{-\bar{m}(r)} \leq 8 / \mathrm{e}$, which is an upper bound for the radius of convergence $R_{0}$. Moreover, from (49) we can derive another consequence for $\bar{m}(x)$ at the border of its domain of convergence: its Taylor expansion is uniformly convergent, and hence, $\bar{m}(x)$ is (at least) a continuous function in the closest disk. This result is also a consequence of the same argument, as if we assume that $\bar{m}(r) \rightarrow+\infty$ when $r \rightarrow R_{0}$, then at the left-hand-side of (49) we obtain that $r \rightarrow 0$, which is also a contradiction. Thus, we have $|\bar{m}(x)| \leq \bar{m}\left(R_{0}\right)<+\infty$ if $|x| \leq R_{0}$.

\subsection{The Newton method in the inner variables}

We recall that our purpose is to prove the existence of an analytic solution $v(\tau)$ for equation (8), in a domain whose lower border is as close as possible to the domain of analyticity of $m(\tau)$, and that, when written as function of $\theta$, overlaps with the domain 
of the solution $u(\theta)$ provided by Corollary 1 . So, once we have proved the existence of a solution of the inner equation (44), it is natural to look for $v(\tau)$ applying again the iterative (Newton) method of Section 2 to equation (8), taking as first iterate $v^{(0)}(\tau)=\tau+m(\tau)$.

From the statement of Theorem 2, it is clear that to ensure convergence we need to know the constants $\mu_{j}^{(0)}, j=1, \ldots, 7$, defined in (39), in this domain. Thus, this method needs that we have a good control of the function $v^{(0)}(\tau)$ and its derivative near the boundary of its domain of analyticity. Lemma 1 give us that $m(\tau)$ is continuous up to the lower border of its strip of analyticity and, due to the fact that it only has positive Fourier harmonics, it is clear that $m(\tau) \rightarrow 0$ when $\operatorname{Im}(\tau) \rightarrow+\infty$. This gives that $\mu_{1}^{(0)}$ is bounded, and $1=a^{(0)}=\mu_{5}^{(0)}=\mu_{6}^{(0)}$. On the other hand, the perturbation term in equation (8) is unbounded when $\operatorname{Im}(\tau) \rightarrow+\infty$. Then, in order to control the constants $\mu_{3}^{(0)}$ and $\mu_{7}^{(0)}$, we have to take an initial domain with a finite upper boundary.

A bigger problem arises when we try to estimate $\mu_{2}^{(0)}$ and $\mu_{4}^{(0)}$. A small reduction of the domain of analyticity of $m(\tau)$ allows us to control the size of $v^{(0)}(\tau)$ by means of the classical Cauchy estimates, obtaining $\mu_{2}^{(0)}$. But since the function $v^{(0)}(\tau)$ can have zeros inside its domain of convergence, we are not able, in principle, to control the constant $\mu_{4}^{(0)}$ inside this domain. Then, for this technical reason, Theorem 2 only can works until $v^{\prime(0)}(\tau)$ reaches its first zero (or until this happens for any iterate $v^{\prime(n)}(\tau)$ ), but it is not reasonable to think on this as a real obstruction for the solution of (8) to exist.

We point out that $v^{\prime}(\tau)$ vaniches (or, thinking in the original variables, if $u^{\prime}(\theta)=0$, with $u(\theta)$ the solution of $(5))$, is not an obstruction to the well defined character of the conjugation. The reason for this is that the invariant curve is given by two components, namely $\left(u_{1}(\theta), u_{2}(\theta)\right)$, where $u_{1}(\theta)=u(\theta)$ and $u_{2}(\theta)=u(\theta+\omega)-u(\theta)-V^{\prime}(u(\theta))$. So, this parameterization is degenerate only when $u_{1}^{\prime}\left(\theta_{0}\right)=u_{2}^{\prime}\left(\theta_{0}\right)=0$ simultaneously, for some $\theta_{0}$, and this happens if and only if $u^{\prime}\left(\theta_{0}\right)=u^{\prime}\left(\theta_{0}+\omega\right)=0$. On the other hand, if $u(\theta)$ is a solution of (5), and $u^{\prime}\left(\theta_{0}\right)=u^{\prime}\left(\theta_{0}+\omega\right)=0$, for certain $\theta_{0}$, and we use that $u^{\prime}(\theta)$ verifies equation (15), then we have that $u^{\prime}(\theta)=0$ in a dense set of values of $\theta$ with $\operatorname{Im}(\theta)=\operatorname{Im}\left(\theta_{0}\right)$, and this can not happens in the domain of analyticity of $u(\theta)$. Nevertheless, a sole zero of $u^{\prime}(\theta)$ (or of $v^{\prime}(\tau)$ ) does not have, in principle, a special meaning, and it seems not reasonable to think that this is a real obstruction for the Newton method of Section 2.2.

Looking more carefully to the method of Section 2.2, one can see that this technical obstruction comes from the way we have constructed the second solution $y(\theta)=u^{\prime}(\theta) c(\theta)=$ $u^{\prime}(\theta)\left(\nu(\theta)+\frac{a}{\omega} \theta\right)$ of the variational equation (15) associated to $u(\theta)$, in the case when $u(\theta)$ is a true solution of (5) (see Section 2.1). Equations (20) and (21) show that the zeros of $u^{\prime}(\theta)$ seems to become singularities for $\nu(\theta)$.

In order to investigate this phenomena, and analogously to the case of second order differential equations, where the simple zeros of the first solution do not cause singularities in the second one, we have that if $u^{\prime}(\theta)$ does not have a couple of zeros with difference equal to $\omega$, then the apparent singularities of $\nu(\theta)$ cancel in the final expression for the second solution $y(\theta)$.

Nevertheless, we can not expect "these cancellations" from the zeros of $u^{\prime}(\theta)$ to singularities of $y(\theta)$, to be also true in Section 2.2, where $y^{(n)}(\theta)$ is defined in the same way than $y(\theta)$ in Section 2.1, but when $u^{(n)}(\theta)$ is not a solution of (5). Thus, it is natural to ask if there is a different way to construct $y^{(n)}(\theta)$, and more concretely, its periodic part $w^{(n)}(\theta)=\nu^{(n)}(\theta) u^{(n)}(\theta)$, not involving additional singularities than the ones coming 
from $u^{(n)}(\theta)$, at least if we shrink a little the domain of analyticity of $u^{(n)}(\theta)$. In the next

section we are going to describe how to formulate a modified iterative method following this way, and its application to equation (8) is given in section 5 . This new method will be also Newton-like (this is, with "quadratic speed" of convergence), and based in the same quasi-reducibility considerations used to construct the sequence $u^{(n)}(\theta)$ of Section 2.2. For this reasons, we will refer to it as the "modified Newton method".

\section{The modified Newton method}

As we have done in section 2.2, to formulate this modified iterative method we consider the "generic case" of (1), and we resume the Lagrangian formulation (5) with an arbitrary entire function $V(q)$ (not necessarily "small") and a fixed Diophantine $\frac{\omega}{2 \pi}$. Let us describe the basic ideas to construct this method.

If we go back to Section 2.1, we recall that if $u(\theta)$ is an exact solution of (5), then the Floquet matrix $P(\theta)(25)$, can be written in terms of $u^{\prime}(\theta)$ and $u^{\prime}(\theta) \nu(\theta)$, which is the periodic part of the second independent solution $y(\theta)$ (16) of the variational equation (15), and that we will call $w(\theta)$. Then, $w(\theta)$ is explicitly given by

$$
w(\theta)=y(\theta)-\frac{a}{\omega} \theta u^{\prime}(\theta)
$$

and verifies the equation

$$
\mathcal{L}_{\omega}^{2} w(\theta)+a\left(u^{\prime}(\theta+\omega)-u^{\prime}(\theta-\omega)\right)=V^{\prime \prime}(u(\theta)) w(\theta) .
$$

Moreover, the Floquet matrix $P(\theta)$ can be written in terms of $u^{\prime}(\theta)$ and $w(\theta)$ as

$$
P(\theta)=\left(\begin{array}{cc}
u^{\prime}(\theta) & w(\theta) \\
\mathcal{L}_{\omega} u^{\prime}(\theta) & \mathcal{L}_{\omega} w(\theta)+a u^{\prime}(\theta+\omega)
\end{array}\right) .
$$

We recall that this matrix verifies that $\operatorname{det}(P(\theta))=1$, and that

$$
\mathcal{L}_{\omega} P(\theta)=A(\theta) P(\theta)-P(\theta+\omega)\left(\begin{array}{ll}
0 & a \\
0 & 0
\end{array}\right),
$$

where $A(\theta)$ is defined in (13). Then, provided with this matrix, in Section 2.1 we have made the periodic change of variables $\zeta=P(\theta) \eta$, which transforms the linear system of difference equations (14) for $\zeta(\theta)$, into equation (27) for $\eta(\theta)$, that is the one we solve when we apply the iterative method of Section 2.2. Now, by using this new notation as function of $w(\theta)$, the non-homogeneous part of (27) can be written as:

$$
(P(\theta+\omega))^{-1}\left(\begin{array}{c}
0 \\
e(\theta+\omega)
\end{array}\right)=\left(\begin{array}{c}
-w(\theta+\omega) \\
u^{\prime}(\theta+\omega)
\end{array}\right) e(\theta+\omega) .
$$

In view of this, the modified Newton method will consists on looking iteratively for $u(\theta)$ and, simultaneously, for $w(\theta)$. Moreover, the iterative computation of $w(\theta)$ will be formulated in terms of the Floquet matrix $P(\theta)$. Thus, we will look iteratively for a $2 \pi$-periodic matrix $P^{(n)}(\theta)$, having the form (52), which solves in an approximate way the corresponding equation (53), with an error that goes to zero "quadratically" as the error of $u^{(n)}(\theta)$. This way to construct $w^{(n)}(\theta)$ has the advantage that, as $\operatorname{det}\left(P^{(n)}(\theta)\right)$ will be close to 1 , the singularities appearing in Section 2.2 due to the vanishing character of $u^{(n)}(\theta)$ will not be present here. 


\subsection{Formulation of the modified iterative method}

Let us start considering $u^{(n)}(\theta)=\theta+\ell^{(n)}(\theta)$ and $w^{(n)}(\theta)$, with $\ell^{(n)}(\theta)$ and $w^{(n)}(\theta)$ analytic and $2 \pi$-periodic in $\theta$, and with $\left\langle\ell^{(n)}\right\rangle=0$. We suppose that both functions are approximate solutions of equations (5) and (51), with errors $e^{(n)}(\theta)$ and $f^{(n)}(\theta)$, respectively. More concretely, we have

$$
\begin{aligned}
e^{(n)}(\theta) & =\mathcal{L}_{\omega}^{2} u^{(n)}(\theta)-V^{\prime}\left(u^{(n)}(\theta)\right) \\
f^{(n)}(\theta) & =\mathcal{L}_{\omega}^{2} w^{(n)}(\theta)+a^{(n)}\left(u^{\prime(n)}(\theta+\omega)-u^{\prime(n)}(\theta-\omega)\right)-V^{\prime \prime}\left(u^{(n)}(\theta)\right) w^{(n)}(\theta) .
\end{aligned}
$$

From $u^{(n)}(\theta)$ and $w^{(n)}(\theta)$ we introduce the approximate Floquet matrix

$$
P^{(n)}(\theta)=\left(\begin{array}{cc}
u^{\prime(n)}(\theta) & w^{(n)}(\theta) \\
\mathcal{L}_{\omega} u^{\prime(n)}(\theta) & \mathcal{L}_{\omega} w^{(n)}(\theta)+a^{(n)} u^{\prime(n)}(\theta+\omega)
\end{array}\right)
$$

where we assume that $a^{(n)} \neq 0$ is given in such a way $P^{(n)}(\theta)$ verifies

$$
\left\langle\operatorname{det}\left(P^{(n)}\right)\right\rangle=1
$$

Let us note that, in section 2.2, the role of $w^{(n)}(\theta)$ was played by $u^{\prime(n)}(\theta) \nu^{(n)}(\theta)$, and the definition $(21)$ of $\nu(\theta)$ implied $\operatorname{det}\left(P^{(n)}(\theta)\right)=1$. Now, with this modified construction, $u^{(n)}(\theta)$ and $w^{(n)}(\theta)$ are given independently and then we can not ask that $\operatorname{det}\left(P^{(n)}(\theta)\right)=1$. What we have in this case is that if we write $\operatorname{det}\left(P^{(n)}(\theta)\right)=1+d^{(n)}(\theta)$, with $\left\langle d^{(n)}\right\rangle=0$, then the size of $d^{(n)}(\theta)$ is of the same order than $e^{(n)}$ and $f^{(n)}$. We can check this using (54) and (55), and computing:

$$
\begin{aligned}
\mathcal{L}_{\omega}\left(\operatorname{det}\left(P^{(n)}(\theta)\right)\right)= & u^{\prime(n)}(\theta+\omega)\left[\mathcal{L}_{\omega}^{2} w^{(n)}(\theta+\omega)+a^{(n)} \mathcal{L}_{\omega} u^{\prime(n)}(\theta+\omega)\right] \\
& +\mathcal{L}_{\omega}\left(u^{\prime(n)}(\theta)\right)\left[\mathcal{L}_{\omega} w^{(n)}(\theta)+a^{(n)} u^{\prime(n)}(\theta+\omega)\right] \\
& -w^{(n)}(\theta+\omega) \mathcal{L}_{\omega}^{2} u^{\prime(n)}(\theta+\omega)-\left(\mathcal{L}_{\omega} w^{(n)}(\theta)\right) \mathcal{L}_{\omega} u^{\prime(n)}(\theta) \\
= & u^{\prime(n)}(\theta+\omega) f^{(n)}(\theta+\omega)-w^{(n)}(\theta+\omega) e^{\prime(n)}(\theta+\omega) .
\end{aligned}
$$

Solving equation (58), we obtain for $d^{(n)}(\theta)$

$$
d^{(n)}(\theta)=\mathcal{L}_{\omega}^{-1}\left[u^{\prime(n)}(\theta+\omega) f^{(n)}(\theta+\omega)-w^{(n)}(\theta+\omega) e^{\prime(n)}(\theta+\omega)\right]
$$

where $\mathcal{L}_{\omega}^{-1}$ is defined in (24). Moreover, if we denote by $E^{(n)}(\theta)$ the error of $P^{(n)}(\theta)$,

$$
E^{(n)}(\theta)=\mathcal{L}_{\omega} P^{(n)}(\theta)-A^{(n)}(\theta) P^{(n)}(\theta)+P^{(n)}(\theta+\omega)\left(\begin{array}{cc}
0 & a^{(n)} \\
0 & 0
\end{array}\right),
$$

equations (54) and (55) give us that

$$
E^{(n)}(\theta)=\left(\begin{array}{cc}
0 & 0 \\
e^{(n)}(\theta+\omega) & f^{(n)}(\theta+\omega)+a^{(n)} e^{(n)}(\theta+\omega)
\end{array}\right) .
$$

Once we have $P^{(n)}(\theta)$, now we can follow the same method explained in Section 2.2 in order to compute $u^{(n+1)}(\theta)=u^{(n)}(\theta)+\xi^{(n)}(\theta)$, where

$$
\xi^{(n)}(\theta)=u^{(n)}(\theta) \eta_{1}^{(n)}(\theta)+w^{(n)}(\theta) \eta_{2}^{(n)}(\theta),
$$


defining the vector $\eta^{(n)}(\theta)=\left(\eta_{1}^{(n)}(\theta), \eta_{1}^{(n)}(\theta)\right), 2 \pi$-periodic in $\theta$, as the solution of the system of difference equations given by

$$
\mathcal{L}_{\omega} \eta^{(n)}(\theta)=\left(\begin{array}{cc}
0 & a^{(n)} \\
0 & 0
\end{array}\right) \eta^{(n)}(\theta)+\left(\begin{array}{c}
w^{(n)}(\theta+\omega) \\
-u^{\prime(n)}(\theta+\omega)
\end{array}\right) e^{(n)}(\theta+\omega),
$$

where as we want $\left\langle\xi^{(n)}\right\rangle=0$, we choose $\left\langle\eta_{1}^{(n)}\right\rangle=-\left\langle w^{(n)} \eta_{2}^{(n)}\right\rangle-\left\langle u^{\prime(n)} \widetilde{\eta}_{1}^{(n)}\right\rangle$ as in (32). Let us recall that the generic conditions needed to solve this system are that $a^{(n)} \neq 0$ and that $\left\langle u^{\prime(n)} e^{(n)}\right\rangle=0$, both fulfilled in this context. Then, as we will see later, the new error $e^{(n+1)}(\theta)$ defined from $(54)$ for $u^{(n+1)}(\theta)$, has quadratic size with respect to the errors $e^{(n)}$ and $f^{(n)}$ of the $n$-step.

Right after we have $u^{(n+1)}(\theta)$, we are going to give a method that computes $w^{(n+1)}(\theta)$ as a small perturbation of $w^{(n)}(\theta)$. The important thing here is that we will look for this small perturbation as a lineal combination of the "old" approximation $w^{(n)}(\theta)$ and the "new" one $u^{\prime(n+1)}(\theta)$. With this construction, we avoid the "extra" singularities appearing if we compute $w^{(n+1)}(\theta)$ only from $u^{(n+1)}(\theta)$.

To compute $w^{(n+1)}(\theta)$ we will proceed as follows. First of all, we consider the following intermediate Floquet matrix between the $n$-step and the $(n+1)$-step:

$$
\bar{P}^{(n)}(\theta)=\left(\begin{array}{cc}
u^{\prime(n+1)}(\theta) & w^{(n)}(\theta) \\
\mathcal{L}_{\omega} u^{\prime(n+1)}(\theta) & \mathcal{L}_{\omega} w^{(n)}(\theta)+a^{(n)} u^{\prime(n+1)}(\theta+\omega)
\end{array}\right) .
$$

This matrix is defined as $P^{(n)}(\theta)$, but replacing $u^{\prime(n)}(\theta)$ by its correction $u^{\prime(n+1)}(\theta)$. The error for this intermediate matrix is

$$
\bar{E}^{(n)}(\theta)=\mathcal{L}_{\omega} \bar{P}^{(n)}(\theta)-A^{(n+1)}(\theta) \bar{P}^{(n)}(\theta)+\bar{P}^{(n)}(\theta+\omega)\left(\begin{array}{cc}
0 & a^{(n)} \\
0 & 0
\end{array}\right),
$$

that using equations (54), for $n+1$, and (55), can be expressed as

$$
\bar{E}^{(n)}(\theta)=\left(\begin{array}{cc}
0 & 0 \\
e^{(n+1)}(\theta+\omega) & g^{(n)}(\theta+\omega)+a^{(n)} e^{(n+1)}(\theta+\omega)
\end{array}\right),
$$

where

$$
\begin{aligned}
g^{(n)}(\theta)= & \mathcal{L}_{\omega}^{2} w^{(n)}(\theta)-V^{\prime \prime}\left(u^{(n+1)}(\theta)\right) w^{(n)}(\theta)+a^{(n)}\left(u^{\prime(n+1)}(\theta+\omega)-u^{\prime(n+1)}(\theta-\omega)\right) \\
= & a^{(n)}\left(\xi^{\prime(n)}(\theta+\omega)-\xi^{\prime(n)}(\theta-\omega)\right)+w^{(n)}(\theta)\left(V^{\prime \prime}\left(u^{(n)}(\theta)\right)-V^{\prime \prime}\left(u^{(n+1)}(\theta)\right)\right) \\
& +f^{(n)}(\theta) .
\end{aligned}
$$

We will check that the first column of $\bar{E}^{(n)}(\theta)$ has quadratic size with respect to $e^{(n)}$ and $f^{(n)}$ (see (79)), but this does not hold for the second one. Then, we have to modify the second column of $\bar{P}^{(n)}(\theta)$, or what it is the same, $w^{(n)}(\theta)$. For this purpose, we will look for $\alpha^{(n)}(\theta)$ and $\beta^{(n)}(\theta), 2 \pi$-periodic in $\theta$, such that if we define

$$
\widehat{w}^{(n)}(\theta)=w^{(n)}(\theta)+\widehat{\chi}^{(n)}(\theta)
$$

with

$$
\widehat{\chi}^{(n)}(\theta)=\alpha^{(n)}(\theta) u^{(n+1)}(\theta)+\beta^{(n)}(\theta) w^{(n)}(\theta)
$$


then we have a quadratic error in equation (55), if we replace $w^{(n)}(\theta)$ by $\widehat{w}^{(n)}(\theta)$ and $u^{(n)}(\theta)$ by $u^{(n+1)}(\theta)$, but keeping the same constant $a^{(n)}$. Let us point that we are not saying that $\widehat{w}^{(n)}(\theta)$ is $w^{(n+1)}(\theta)$, because if we define $P^{(n+1)}(\theta)$ from $u^{(n+1)}$ and $\widehat{w}^{(n)}$, we can not ensure that the condition $\left\langle\operatorname{det}\left(P^{(n+1)}\right)\right\rangle=1$ is fulfilled. This is because we compute $\widehat{w}^{(n)}(\theta)$ working with the same $a^{(n)}$ than in the $n$-step. Then, once we have computed $\widehat{w}^{(n)}$, we will be forced to done a suitable scaling (see (77)) in order to have $\left\langle\operatorname{det}\left(P^{(n+1)}\right)\right\rangle=1$.

To obtain the equations for $\left(\alpha^{(n)}(\theta), \beta^{(n)}(\theta)\right)$, we formulate the problem in compact (matrix) way, looking for a square matrix $B^{(n)}(\theta), 2 \pi$-periodic in $\theta$, such that $\bar{P}^{(n)}(\theta) B^{(n)}(\theta)$ verifies the Floquet equation (53) for $A^{(n+1)}(\theta)$. Using (65), one obtains the following equation for $B^{(n)}(\theta)$ :

$\mathcal{L}_{\omega} B^{(n)}(\theta)=\left(\begin{array}{cc}0 & a^{(n)} \\ 0 & 0\end{array}\right) B^{(n)}(\theta)-B^{(n)}(\theta+\omega)\left(\begin{array}{cc}0 & a^{(n)} \\ 0 & 0\end{array}\right)-\left(\bar{P}^{(n)}(\theta+\omega)\right)^{-1} \bar{E}^{(n)}(\theta) B^{(n)}(\theta)$.

As we can not solve this equation, we will choose $B^{(n)}(\theta)$ to solve it except by a "quadratic" error. Thus, from the discussions before, it is natural to look for

$$
B^{(n)}(\theta)=\left(\begin{array}{cc}
1 & \alpha^{(n)}(\theta) \\
0 & 1+\beta^{(n)}(\theta)
\end{array}\right)
$$

The error of the first column it is clearly squared, and for the second column, if we skip the terms having quadratic size, we have

$$
\mathcal{L}_{\omega}\left(\begin{array}{c}
\alpha^{(n)}(\theta) \\
\beta^{(n)}(\theta)
\end{array}\right)=\left(\begin{array}{c}
a^{(n)} \beta^{(n)}(\theta) \\
0
\end{array}\right)+\left(\begin{array}{c}
w^{(n)}(\theta+\omega) g^{(n)}(\theta+\omega) \\
-u^{(n+1)}(\theta+\omega) g^{(n)}(\theta+\omega)
\end{array}\right) .
$$

In order to obtain this equation, we have used that, $\operatorname{det}\left(\bar{P}^{(n)}(\theta)\right)$ and $\operatorname{det}\left(P^{(n)}(\theta)\right)$ are close to 1 except by terms of order of the error of the $n$-step. Unfortunately, we can not ensure that the second component of the non-homogeneous part of (71) has zero average. In fact, we have that:

$$
\begin{aligned}
u^{\prime(n+1)}(\theta) g^{(n)}(\theta)= & u^{\prime(n+1)}(\theta) \mathcal{L}_{\omega}^{2} w^{(n)}(\theta)+e^{\prime(n+1)}(\theta) w^{(n)}(\theta)-w^{(n)}(\theta) \mathcal{L}_{\omega}^{2} u^{\prime(n+1)}(\theta) \\
& +a^{(n)}\left(u^{\prime(n+1)}(\theta+\omega)-u^{\prime(n+1)}(\theta-\omega)\right) u^{\prime(n+1)}(\theta)
\end{aligned}
$$

where we have used (67) and

$$
e^{\prime(n+1)}(\theta)=\mathcal{L}_{\omega}^{2} u^{\prime(n+1)}(\theta)-V^{\prime \prime}\left(u^{(n+1)}(\theta)\right) u^{\prime(n+1)}(\theta),
$$

which is obtained taking derivatives in equation (54) for $n+1$. Now, proceeding as in $(30)$, we can see that $u^{\prime(n+1)}(\theta) g^{(n)}(\theta)-e^{\prime(n+1)}(\theta) w^{(n)}(\theta)$ has zero average, and as $e^{\prime(n+1)}(\theta) w^{(n)}(\theta)$ is only a correction with "quadratic size", we can replace equation (71) for $\left(\alpha^{(n)}, \beta^{(n)}\right)$ by

$$
\begin{aligned}
\mathcal{L}_{\omega}\left(\begin{array}{c}
\alpha^{(n)}(\theta) \\
\beta^{(n)}(\theta)
\end{array}\right)= & \left(\begin{array}{c}
a^{(n)} \beta^{(n)}(\theta) \\
0
\end{array}\right) \\
& +\left(\begin{array}{c}
w^{(n)}(\theta+\omega) g^{(n)}(\theta+\omega) \\
e^{\prime(n+1)}(\theta+\omega) w^{(n)}(\theta+\omega)-u^{\prime(n+1)}(\theta+\omega) g^{(n)}(\theta+\omega)
\end{array}\right) \cdot
\end{aligned}
$$


Then, we can solve (73) choosing, for instance, $\alpha^{(n)}(\theta)$ with zero average, and formula (69) gives us $\widehat{\chi}^{(n)}(\theta)$, and hence, $\widehat{w}^{(n)}(\theta)$. Before continue, we define $\widehat{f}^{(n)}(\theta)$ as the error of $\widehat{w}^{(n)}(\theta)$, and $\widehat{P}^{(n)}(\theta)$ the "corresponding" Floquet matrix. More concretely, we have

$$
\widehat{f}^{(n)}(\theta)=\mathcal{L}_{\omega}^{2} \widehat{w}^{(n)}(\theta)+a^{(n)}\left(u^{\prime(n+1)}(\theta+\omega)-u^{\prime(n+1)}(\theta-\omega)\right)-V^{\prime \prime}\left(u^{(n+1)}(\theta)\right) \widehat{w}^{(n)}(\theta)
$$

and

$$
\widehat{P}^{(n)}(\theta)=\left(\begin{array}{cc}
u^{\prime(n+1)}(\theta) & \widehat{w}^{(n)}(\theta) \\
\mathcal{L}_{\omega} u^{\prime(n+1)}(\theta) & \mathcal{L}_{\omega} \widehat{w}^{(n)}(\theta)+a^{(n)} u^{\prime(n+1)}(\theta+\omega)
\end{array}\right) .
$$

Now, to end the formulation of this modified method, we only have to define $w^{(n+1)}(\theta)$ and $a^{(n+1)}$ scaling $\widehat{w}^{(n)}(\theta)$ and $a^{(n)}$. To do this, we note that

$$
\operatorname{det}\left(\widehat{P}^{(n)}(\theta)\right)=\operatorname{det}\left(P^{(n)}(\theta)\right)+\Delta \widehat{d}^{(n)}(\theta),
$$

where

$$
\begin{aligned}
\Delta \widehat{d}^{(n)}(\theta)= & \xi^{\prime(n)}(\theta) \mathcal{L}_{\omega} \widehat{w}^{(n)}(\theta)+u^{\prime(n)}(\theta) \mathcal{L}_{\omega} \widehat{\chi}^{(n)}(\theta) \\
& -\widehat{\chi}^{(n)}(\theta) \mathcal{L}_{\omega} u^{\prime(n+1)}(\theta)-\widehat{w}^{(n)}(\theta) \mathcal{L}_{\omega} \xi^{\prime(n)}(\theta) \\
& +a^{(n)}\left(\xi^{\prime(n)}(\theta+\omega) u^{\prime(n)}(\theta)+\xi^{\prime(n)}(\theta) u^{\prime(n+1)}(\theta+\omega)\right) .
\end{aligned}
$$

Then, by (57) we have that

$$
\left\langle\operatorname{det}\left(\widehat{P}^{(n)}\right)\right\rangle=1+\left\langle\Delta \widehat{d}^{(n)}\right\rangle
$$

and we can define the new iteration as

$$
w^{(n+1)}(\theta)=\frac{1}{1+\left\langle\Delta \widehat{d}^{(n)}\right\rangle} \widehat{w}^{(n)}(\theta), \quad a^{(n+1)}=\frac{1}{1+\left\langle\Delta \widehat{d}^{(n)}\right\rangle} a^{(n)},
$$

and the corresponding Floquet matrix for the $(n+1)$-step

$$
P^{(n+1)}(\theta)=\left(\begin{array}{cc}
u^{\prime(n+1)}(\theta) & w^{(n+1)}(\theta) \\
\mathcal{L}_{\omega} u^{\prime(n+1)}(\theta) & \mathcal{L}_{\omega} w^{(n+1)}(\theta)+a^{(n+1)} u^{\prime(n+1)}(\theta+\omega)
\end{array}\right) .
$$

It is clear that the new iteration verifies:

$\mathcal{L}_{\omega}^{2} \widehat{w}^{(n+1)}(\theta)+a^{(n+1)}\left(u^{\prime(n+1)}(\theta+\omega)-u^{\prime(n+1)}(\theta-\omega)\right)=V^{\prime \prime}\left(u^{(n+1)}(\theta)\right) w^{(n+1)}(\theta)+f^{(n+1)}(\theta)$,

with

$$
f^{(n+1)}(\theta)=\frac{1}{1+\left\langle\Delta \widehat{d}^{(n)}\right\rangle} \widehat{f}^{(n)}(\theta)
$$

\subsection{Analysis of the errors in the modified iterative method}

Right after we have introduced the modified Newton method, in this section we want to give explicit expressions of the new errors $e^{(n+1)}(\theta)$ and $f^{(n+1)}(\theta)$ in terms of the errors in the previous step. First of all, we study the error of $u^{(n+1)}(\theta)$. As we have done in (34), the error of $u^{(n+1)}(\theta)$ can be decomposed as a sum of $e_{2}^{(n+1)}(\theta)$, the error due to the Newton method, and $e_{1}^{(n+1)}(\theta)$, the error due to the quasi-reducibility. The considerations about $e_{2}^{(n+1)}(\theta)$ are the same explained in Section 2.2, and a explicit formula is given in (34). 
In order to give $e_{1}^{(n+1)}(\theta)$ in this context, we can repeat the computations that lead to formula (36), but now, we have to use that the error of the Floquet matrix $P^{(n)}(\theta)$ is given by (60) and (61) instead of (35), obtaining

$$
e_{1}^{(n+1)}(\theta)=e^{\prime(n)}(\theta) \eta_{1}^{(n)}(\theta-\omega)+\left(f^{(n)}(\theta)+a^{(n)} e^{\prime(n)}(\theta)\right) \eta_{2}^{(n)}(\theta-\omega)
$$

The next step is to study the error $\widehat{E}^{(n)}(\theta)$ of the matrix $\bar{P}^{(n)}(\theta) B^{(n)}(\theta)$, where $B^{(n)}(\theta)$ is given by (70). More concretely, calling $\widehat{E}_{2}^{(n)}(\theta)$ the error of its second column:

$$
\begin{aligned}
\widehat{E}_{2}^{(n)}(\theta)= & \mathcal{L}_{\omega}\left[\bar{P}^{(n)}(\theta)\left(\begin{array}{c}
\alpha^{(n)}(\theta) \\
1+\beta^{(n)}(\theta)
\end{array}\right)\right]-A^{(n+1)}(\theta)\left[\bar{P}^{(n)}(\theta)\left(\begin{array}{c}
\alpha^{(n)}(\theta) \\
1+\beta^{(n)}(\theta)
\end{array}\right)\right] \\
& +\bar{P}^{(n)}(\theta+\omega) B(\theta+\omega)\left(\begin{array}{c}
a^{(n)} \\
0
\end{array}\right),
\end{aligned}
$$

we can compute this error using equations (65) and (73), obtaining

$$
\begin{aligned}
\hat{E}_{2}^{(n)}(\theta)= & \bar{E}^{(n)}(\theta)\left(\begin{array}{c}
\alpha^{(n)}(\theta) \\
1+\beta^{(n)}(\theta)
\end{array}\right) \\
& +\bar{P}^{(n)}(\theta+\omega)\left(\begin{array}{c}
w^{(n)}(\theta+\omega) g^{(n)}(\theta+\omega) \\
-u^{(n+1)}(\theta+\omega) g^{(n)}(\theta+\omega)+w^{(n)}(\theta+\omega) e^{\prime(n+1)}(\theta+\omega)
\end{array}\right) .
\end{aligned}
$$

Moreover, using (64) and (66), we have after some computations:

$$
\widehat{E}_{2}^{(n)}(\theta)=\left(\begin{array}{c}
\widehat{e}_{1}^{(n)}(\theta) \\
\widehat{e}_{2}^{(n)}(\theta)
\end{array}\right)
$$

with

$$
\begin{aligned}
\widehat{e}_{1}^{(n)}(\theta)= & \left(w^{(n)}(\theta+\omega)\right)^{2} e^{(n+1)}(\theta+\omega), \\
\hat{e}_{2}^{(n)}(\theta)= & e^{\prime(n+1)}(\theta+\omega)\left[\alpha^{(n)}(\theta)+a^{(n)}\left(1+\beta^{(n)}(\theta)+u^{\prime(n+1)}(\theta+2 \omega) w^{(n)}(\theta+\omega)\right)\right. \\
& \left.+w^{(n)}(\theta+\omega) \mathcal{L}_{\omega} w^{(n)}(\theta+\omega)\right]+g^{(n)}(\theta+\omega)\left[1-\operatorname{det}\left(\bar{P}^{(n)}(\theta+\omega)\right)+\beta^{(n)}(\theta)\right] .
\end{aligned}
$$

In order to control $\hat{e}_{1}^{(n)}(\theta)$ and $\hat{e}_{2}^{(n)}(\theta)$ we note that

$$
1-\operatorname{det}\left(\bar{P}^{(n)}(\theta)\right)=1-\operatorname{det}\left(P^{(n)}(\theta)\right)+\operatorname{det}\left(\bar{P}^{(n)}(\theta)\right)-\operatorname{det}\left(P^{(n)}(\theta)\right)=-d^{(n)}(\theta)+\Delta \bar{d}^{(n)}(\theta),
$$

where $d^{(n)}(\theta)$ is defined in (59) and

$$
\begin{aligned}
\Delta \bar{d}^{(n)}(\theta)= & \operatorname{det}\left(\bar{P}^{(n)}(\theta)\right)-\operatorname{det}\left(P^{(n)}(\theta)\right) \\
= & \xi^{\prime(n)}(\theta) \mathcal{L}_{\omega} w^{(n)}(\theta)-w^{(n)}(\theta) \mathcal{L}_{\omega} \xi^{\prime(n)}(\theta) \\
& +a^{(n)}\left[u^{\prime(n)}(\theta) \xi^{\prime(n)}(\theta+\omega)+\xi^{\prime(n)}(\theta) u^{\prime(n+1)}(\theta+\omega)\right] .
\end{aligned}
$$

It is clear from these expressions that both, $\widehat{e}_{1}^{(n)}(\theta)$ and $\widehat{e}_{2}^{(n)}(\theta)$, have quadratic size. 
To control the size of $\widehat{f}^{(n)}(\theta)$ (see (74)), we write explicitly equation (80), component by component, and we use the definition of $\widehat{w}^{(n)}(\theta)$ from (68) and (69), having

$$
\begin{aligned}
\widehat{e}_{1}^{(n)}(\theta)= & \mathcal{L}_{\omega} \widehat{w}^{(n)}(\theta)-\left(\mathcal{L}_{\omega} u^{\prime(n+1)}(\theta)\right) \alpha^{(n)}(\theta) \\
& -\left[\mathcal{L}_{\omega} w^{(n)}(\theta)+a^{(n)} u^{\prime(n+1)}(\theta+\omega)\right]\left(1+\beta^{(n)}(\theta)\right)+a^{(n)} u^{\prime(n+1)}(\theta+\omega), \\
\widehat{e}_{2}^{(n)}(\theta)= & \mathcal{L}_{\omega}\left[\left(\mathcal{L}_{\omega} u^{\prime(n+1)}(\theta)\right) \alpha^{(n)}(\theta)+\left(\mathcal{L}_{\omega} w^{(n)}(\theta)+a^{(n)} u^{\prime(n+1)}(\theta+\omega)\right)\left(1+\beta^{(n)}(\theta)\right)\right] \\
& -V^{\prime \prime}\left(u^{(n+1)}(\theta+\omega)\right)\left[\widehat{w}^{(n)}(\theta)+\left(\mathcal{L}_{\omega} u^{\prime(n+1)}(\theta)\right) \alpha^{(n)}(\theta)\right. \\
& \left.+\left(\mathcal{L}_{\omega} w^{(n)}(\theta)+a^{(n)} u^{\prime(n+1)}(\theta+\omega)\right)\left(1+\beta^{(n)}(\theta)\right)\right]+a^{(n)} \mathcal{L}_{\omega} u^{(n+1)}(\theta+\omega) .
\end{aligned}
$$

Joining these two equations, we obtain:

$$
\begin{aligned}
\hat{e}_{2}^{(n)}(\theta)= & \mathcal{L}_{\omega}\left[\mathcal{L}_{\omega} \widehat{w}^{(n)}(\theta)+a^{(n)} u^{\prime(n+1)}(\theta+\omega)-\widehat{e}_{1}^{(n)}(\theta)\right] \\
& -V^{\prime \prime}\left(u^{(n+1)}(\theta+\omega)\right)\left[\widehat{w}^{(n)}(\theta+\omega)+a^{(n)} u^{(n+1)}(\theta+\omega)-\widehat{e}_{1}^{(n)}(\theta)\right] \\
& +a^{(n)} \mathcal{L}_{\omega} u^{\prime(n+1)}(\theta+\omega) .
\end{aligned}
$$

Now, using (72), we have

$$
\begin{aligned}
\mathcal{L}_{\omega}^{2} \widehat{w}^{(n)}(\theta+\omega)+a^{(n)}\left(u^{\prime(n+1)}(\theta+2 \omega)-u^{\prime(n+1)}(\theta)\right)= & V^{\prime \prime}\left(u^{(n+1)}(\theta+\omega)\right) \widehat{w}^{(n)}(\theta+\omega) \\
& +\widehat{f}^{(n)}(\theta+\omega),
\end{aligned}
$$

with

$$
\widehat{f}^{(n)}(\theta)=\mathcal{L}_{\omega} \widehat{e}_{1}^{(n)}(\theta-\omega)-a^{(n)} e^{(n+1)}(\theta)+\widehat{e}_{2}^{(n)}(\theta-\omega)-V^{\prime \prime}\left(u^{(n+1)}(\theta)\right) \widehat{e}_{1}^{(n)}(\theta-\omega) .
$$

\section{The standard map as a singular perturbation of the semi-standard map}

As we explained in Section 3, we want to apply the modified Newton method, formulated in Section 4, to equation (8). This equation corresponds to equation (5) with $\omega=2 \pi \gamma$, in the case of the standard map, written in the inner variables. Thus, we have to consider the equation

$$
\mathcal{L}_{\omega}^{2} v(\tau)=V^{\prime}(v(\tau))
$$

where $V(q)=\frac{1}{2} e^{i q}+\frac{1}{2} \varepsilon^{2} e^{-i q}$ and to look for $v(\tau)=\tau+l(\tau)$, with $l(\tau) 2 \pi$-periodic in $\tau$ and with zero average. As first approximation for the solution of (85), we will take $v^{(0)}(\tau)=\tau+m(\tau)$, where $m(\tau)$ is the solution of the inner equation (44), which corresponds to $(85)$ for $\varepsilon=0$. As $w^{(0)}(\tau)$, we will take the periodic part (50) of the solution of the variational equation around $v^{(0)}(\tau)$ associated to the inner equation (44).

We recall that in Section 3.1. we have proved that $m(\tau)$ is defined in $\mathcal{D}\left(-\log R_{0},+\infty\right)$, which is the strip of convergence of its Fourier expansion, and hence, the domain of definition of $v^{(0)}(\tau)$. Of course, when doing a perturbative analysis of the solution of (85), we can not expect to keep an infinite upper bound for the domain of analyticity of $v(\tau)$, as $V^{\prime}(q)$ becomes unbounded when $\operatorname{Im}(q) \rightarrow+\infty$. But we recall that our purpose is a bit less ambitious: what we want to establish is that the solution of (85) is defined in a domain whose lower boundary is asymptotically close, when $\varepsilon \rightarrow 0$, to the lower boundary 
$-\log R_{0}$ of the domain of analyticity of $v^{(0)}(\tau)$, and whose upper boundary matches, when written in the $\theta$ variables (see (6)), with the domain of analyticity of the solution of (5), obtained in Corollary 1 . Then, we obtain that the solution $v(\tau)$ of $(85)$ is the analytic continuation of the solution $u(\theta)$ of $(5)$.

In Section 3.2 we have discussed why the formulation of the Newton method done in Section 2 is not suitable to achieve this objective. Now, we want to see that by using the modified formulation, we can overcome the previous difficulties. In order to apply this method, we need some information referring to $v^{(0)}(\tau)$, which can be obtained from the properties of $m(\tau)$ (see Lemma 1). Moreover, we also need additional considerations referring to $w^{(0)}(\tau)$ to be able to proceed with the modified Newton method. This is done in the following section.

\subsection{The variational equation of the inner solution}

Let us consider the variational equation of $(44)$ around $v^{(0)}(\tau)$

$$
\mathcal{L}_{\omega}^{2}(z(\tau))=-\frac{1}{2} e^{i(\tau+m(\tau))} z(\tau)
$$

It is clear that $z(\tau) \equiv v^{\prime(0)}(\tau)=1+m^{\prime}(\tau)$ is a solution of (86). As we have seen in Section 2.1, we can look for an independent solution of (86) of the form

$$
z^{(0)}(\tau)=\frac{1}{\omega} v^{(0)}(\tau) \tau+w^{(0)}(\tau)
$$

where $w^{(0)}(\tau)$ is $2 \pi$-periodic in $\tau$, with zero average, and verifies (see equation (51))

$$
\mathcal{L}_{\omega}^{2}\left(w^{(0)}(\tau)\right)+m^{\prime}(\tau+\omega)-m^{\prime}(\tau-\omega)=-\frac{1}{2} e^{i(\tau+m(\tau))} w^{(0)}(\tau)
$$

In order to study the analyticity properties of $w^{(0)}(\tau)$, and in analogous way as we have done in Section 3.1 to study $m(\tau)$, we replace the Fourier expansion of $w^{(0)}(\tau)$ (which is immediate to check that only contains positive Fourier harmonics) by a Taylor expansion in the variable $x \equiv \mathrm{e}^{i \tau}$. Then, we define $\bar{w}(x) \equiv i w^{(0)}(\tau)$, that can be written as

$$
\bar{w}(x)=\sum_{k \geq 1} w_{k} x^{k}
$$

and verifies

$$
\bar{w}(x \Omega)-2 \bar{w}(x)+\bar{w}(x / \Omega)+i\left(x \Omega \bar{m}^{\prime}(x \Omega)-\frac{x}{\Omega} \bar{m}^{\prime}(x / \Omega)\right)=-\frac{x}{2} \mathrm{e}^{\bar{m}(x)} \bar{w}(x),
$$

where $\bar{m}(x)$ has been defined in $(45)$ and $\Omega=\mathrm{e}^{i \omega}$. From this expression, it is straightforward to obtain the following recurrence for the coefficients $w_{k}$ :

$$
w_{k+1}=\frac{1}{4 D_{k+1}} \sum_{j=1}^{k} F_{k-j} w_{j}-(k+1) m_{k+1} \frac{\cos \left(\frac{(k+1) \omega}{2}\right)}{\sin \left(\frac{(k+1) \omega}{2}\right)},
$$

for any $k \geq 0$. The expressions $m_{k}, F_{k}$ and $D_{k}$ have been introduced in Section 3.1. From (88), and using that $m_{k}, F_{k}$ and $D_{k}$ are real numbers one obtain that $w_{k} \in \mathbb{R}$ for any $k$.

Now, to continue studying $\bar{w}(x)$, the most important point is to relate the radii of convergence of $\bar{m}(x)$ and $\bar{w}(x)$ (or in equivalent way, the strips of analyticity of $m(\tau)$ and $\left.w^{(0)}(\tau)\right)$. What we are going to prove is that the radius of convergence of $\bar{w}(x)$ is bigger or equal to the one of $\bar{m}(x)$. 
Lemma 2 Assuming all the previous notations and definitions, we have

$$
\left|w_{k}\right| \leq M_{1} k^{2} \log (k+1) m_{k}, \quad k \geq 1,
$$

for certain $M_{1}>0$ (independent of $k$ ).

Proof: We consider the recurrence (88), and we divide it by $m_{k+1}>0$, obtaining

$$
\frac{\left|w_{k+1}\right|}{m_{k+1}} \leq \frac{1}{4 D_{k+1} m_{k+1}} \sum_{j=1}^{k}\left(j F_{k-j} m_{j} \frac{\left|w_{j}\right|}{j m_{j}}\right)+\frac{(k+1)}{\left|\sin \left(\frac{(k+1) \omega}{2}\right)\right|}
$$

Now, let us assume for a moment that $\frac{\left|w_{j}\right|}{j m_{j}}$ is an increasing function of $j$. If this were true, we would have

$$
\frac{\left|w_{k+1}\right|}{m_{k+1}} \leq \frac{\left|w_{k}\right|}{m_{k}}+\frac{(k+1)}{\left|\sin \left(\frac{(k+1) \omega}{2}\right)\right|}
$$

where we have used (47) and (48). Of course, the previous assertion on the monotone character of $\frac{\left|w_{j}\right|}{j m_{j}}$ has not to be fulfilled. To overcome this problem we define the following auxiliary recurrence:

$$
\mathcal{W}_{k+1}=\frac{(k+1)}{k} \mathcal{W}_{k}+\frac{(k+1)}{\left|\sin \left(\frac{(k+1) \omega}{2}\right)\right|}
$$

with $\mathcal{W}_{1}=\frac{\left|w_{1}\right|}{m_{1}}$. Then, it is straightforward to prove by induction that $\frac{\left|w_{k}\right|}{m_{k}} \leq \mathcal{W}_{k}$. Moreover, from its recursive definition, we derive the following explicit expression for $\mathcal{W}_{k}$ :

$$
\mathcal{W}_{k}=k \mathcal{W}_{1}+k \sum_{j=2}^{k} \frac{1}{\left|\sin \left(\frac{j \omega}{2}\right)\right|}, \quad k \geq 1 \text {. }
$$

To estimate the coefficients $\mathcal{W}_{k}$, we have to bound $\left|\sin \left(\frac{j \omega}{2}\right)\right|^{-1}$. For this purpose we define, for any $j \in \mathbb{N}, J(j)=\operatorname{int}\left(j \gamma+\frac{1}{2}\right)$, where int $(\cdot)$ states for the integer part of a real number. Let us point that $J(j)$ is the unique $l \in \mathbb{N}$ such that

$$
-\frac{\pi}{2} \leq \frac{j \omega}{2}-l \pi<\frac{\pi}{2}
$$

Then, as we have that

we obtain

$$
|\sin t| \geq \frac{2}{\pi}|t|, \quad t \in\left[-\frac{\pi}{2}, \frac{\pi}{2}\right]
$$

$$
\mathcal{W}_{k} \leq k \mathcal{W}_{1}+\frac{k}{2} \sum_{j=2}^{k} \frac{1}{|j \gamma-J(j)|}
$$

and using that $\omega=2 \pi \gamma$ verifies the Diophantine condition (4) with $\sigma=2$, for certain constant $c_{\omega} \equiv c_{\gamma}>0$, we derive that

$$
\mathcal{W}_{k} \leq k \mathcal{W}_{1}+\frac{k}{4 c_{\gamma}}\left(k^{2}+k-2\right)
$$

which gives that $\mathcal{W}_{k}$, and then $\frac{\left|w_{k}\right|}{m_{k}}$, are bounded by an expression of $\mathcal{O}\left(k^{3}\right)$, proving the "equality" between both radii. But this estimate can been improved, as not for any $j$ in 
the sum (89) we have that $|j \gamma-J(j)|$ is as small as the Diophantine condition gives. In fact, this rough estimate can be improved, giving us that $\mathcal{W}_{k}$ is bounded by an expression of order $\mathcal{O}\left(k^{2} \log (k+1)\right)$, which proves the bound claimed in the statement. This fact comes immediately from the result given in Lemma 8.

Provided with this result, we are in conditions to estimate the size of $\bar{w}(x)$ close to its radius of convergence, or, in equivalent way, the size of $w^{(0)}(\tau)$ close to the boundary of its strip of analyticity.

Lemma 3 For any $\delta>0$,

$$
\left\|w^{(0)}(\tau)\right\|_{-\log R_{0}+\delta,+\infty} \leq M_{2} \mathrm{e}^{-\delta}\left(\frac{\log (1 / \delta)}{\delta^{2}}+1\right),
$$

where $M_{2}>0$ is independent of $\delta$.

Proof: From Lemma 2, we have that

$$
\left\|w^{(0)}(\tau)\right\|_{-\log R_{0}+\delta,+\infty} \leq M_{1} \sum_{k \geq 1} k^{2} \log (k+1) m_{k} R_{0}^{k} \mathrm{e}^{-\delta k} \equiv M_{1} f(\delta),
$$

then, we only need to bound $f(\delta)$. To do that, let us take $\delta>0$ fixed, and let us define for $t \geq 1$

$$
g_{\delta}(t)=t^{2} \log (t+1) \mathrm{e}^{-\delta t} .
$$

Then, we have that

$$
f(\delta) \leq\left(\sup _{t \geq 1} g_{\delta}(t)\right) \sum_{k \geq 1} m_{k} R_{0}^{k}
$$

where we recall the convergent character of the sum above (see Lemma 1 ). In order to bound this expression, we look for the zeros of $g_{\delta}^{\prime}(t)$, with $t \geq 1$. We obtain for these zeros the equation $h(t)=\delta$, where

$$
h(t)=\frac{2}{t}+\frac{1}{(t+1) \log (t+1)} .
$$

We notice that $h(t)$ is well defined for any $t>0$, and it is strictly decreasing with $t$. If we define $\delta_{0}=h(1)=2+\frac{1}{2 \log 2}$, it is immediately to check that if $\delta \geq \delta_{0}$, then $g_{\delta}(t) \leq \mathrm{e}^{-\delta} \log 2$. If $\delta<\delta_{0}$, we have that $h(2 / \delta)>\delta$, and $h(4 / \delta)<\delta$. Hence, it is clear that, in this case, the only value of $t \geq 1$ given rise to the maximum of $g_{\delta}(t)$, is between $2 / \delta$ and $4 / \delta$. This allows to obtain the estimate $g_{\delta}(t) \leq \frac{16}{\delta^{2}} \log \left(\frac{4}{\delta}+1\right) \mathrm{e}^{-2 \delta}$, if $\delta<\delta_{0}$.

As both behaviours, when $\delta \rightarrow 0$ or when $\delta \rightarrow+\infty$ are completely different, it is not easier to found a compact expression matching them uniformly. But it is straightforward to check that the expression given in the statement plays the desired role.

\subsection{Convergence of the modified Newton method}

In this section we are going to prove that the modified Newton method of Section 4 can be applied to equation (85), giving a sequence $v^{(n)}(\tau)$ which converges to the solution $v(\tau)$ in a suitable domain, and that in this domain it is a small perturbation of the solution $v^{(0)}(\tau)$ corresponding to the unperturbed case $\varepsilon=0$. 
As it has been seen in Section 3.1, the solution for $\varepsilon=0$ (the inner equation) is given by $v^{(0)}(\tau)=\tau+l^{(0)}(\tau)$, with $l^{(0)}(\tau)=m(\tau)$, where $m(\tau)$, defined as the periodic solution of (44), is convergent if $\operatorname{Im}(\tau) \geq-\log R_{0}$ (see Lemma 1). On the other hand, from Lemma 3 we have that if we make a small reduction of the lower border of the domain of $m(\tau)$, then $w^{(0)}(\tau)$, defined as the periodic solution of equation (87), exists and it is bounded. Nevertheless, the error associated to $v^{(0)}(\tau)$, if we ask it to verify equation (85), increases unboundedly when $\operatorname{Im}(\tau) \rightarrow+\infty$. So, it is clear that we have to restrict the upper boundary of the domain by taking a finite border. Then, in order to proceed with the modified iterative method, let us consider the following initial domain $D^{(0)}$ for the variable $\tau$ :

$$
D^{(0)}=\mathcal{D}\left(\rho_{1}^{(0)}, \rho_{2}^{(0)}\right)=\left\{\tau \in \mathbb{C}: \rho_{1}^{(0)} \leq \operatorname{Im}(\tau) \leq \rho_{2}^{(0)}\right\}
$$

where we take

$$
\rho_{1}^{(0)}=-\log R_{0}+\delta^{(0)}, \quad \rho_{2}^{(0)}=\log B .
$$

Here we can allow, in principle, any arbitrary $B>1 / R_{0}$ and any $0<\delta^{(0)}<\log B+\log R_{0}$. Nevertheless, and for technical reasons, we will add another restrictions to $B$ and $\delta^{(0)}$. Let us point that even though $v^{(0)}(\tau)$ and $w^{(0)}(\tau)$ are bounded in $D^{(0)}$, the estimate (90) for the norm of $w^{(0)}(\tau)$ grows unboundedly when $\delta^{(0)} \rightarrow 0$. But we recall that our purpose is to prove convergence of the method for $\delta^{(0)}$ as small as possible, in order to establish the existence of the solution of equation (85) in a domain as close as possible to the initial one. This forces us to control the asymptotic behaviour of $w^{(0)}(\tau)$ as function of the distance to the lower boundary. Moreover, this control has to be extended to the successive iterates $w^{(n)}(\tau)$, as well as to other functions that will appear in the sequel (for instance, $v^{\prime(0)}(\tau)$ ). To do that, we introduce the following notation for the weighted norm (38) of a $2 \pi$-periodic analytic function $f(\tau)$ :

$$
\|f(\tau)\|_{D^{(0)}-\delta}=\|f(\tau)\|_{\rho_{1}^{(0)}+\delta, \rho_{2}^{(0)}-\delta},
$$

for any $0 \leq \delta \leq\left(\rho_{2}^{(0)}-\rho_{1}^{(0)}\right) / 2$. Expressing the norm as a function of $\delta$, we can describe the size of $f(\tau)$ as a function of the distance to the boundary of $D^{(0)}$, and hence, we can manage with quantitative estimates for the asymptotic behaviour of this norm for different values of $\delta$.

Provided with these notations, and using the analysis that we have made in Section 3.1, bound (90) and the Cauchy estimates for the derivatives, we get that there exists a constant $M \geq 1$, independent of $\varepsilon, \delta, \delta^{(0)}$ and $B$, such that:

$$
\begin{aligned}
\left\|l^{(0)}(\tau)\right\|_{D^{(0)}-\delta} & \leq M \mathrm{e}^{-\left(\delta+\delta^{(0)}\right)}, \quad\left\|v^{(0)}(\tau)\right\|_{D^{(0)}-\delta} \leq \frac{M}{\delta^{(0)}+\delta}+1 \\
\left\|w^{(0)}(\tau)\right\|_{D^{(0)}-\delta} & \leq M \mathrm{e}^{-\left(\delta+\delta^{(0)}\right)}\left(\frac{\log \left(\frac{1}{\delta^{(0)}+\delta}\right)}{\left(\delta^{(0)}+\delta\right)^{2}}+1\right), \\
\left\|e^{(0)}(\tau)\right\|_{D^{(0)}-\delta} & \leq M B \varepsilon^{2}, \quad\left\|f^{(0)}(\tau)\right\|_{D^{(0)}-\delta} \leq M B \varepsilon^{2} \mathrm{e}^{-\left(\delta+\delta^{(0)}\right)}\left(\frac{\log \left(\frac{1}{\delta^{(0)}+\delta}\right)}{\left(\delta^{(0)}+\delta\right)^{2}}+1\right),
\end{aligned}
$$

where $e^{(0)}(\tau)$ and $f^{(0)}(\tau)$ are defined in (54) and (55), respectively.

To state Theorem 3, in analogous to Theorem 2 (see (39)), first of all we have to introduce several constants to control the size of the objects involved in the proof. For 
the initial approximation we use

$$
\begin{aligned}
\left\|l^{(0)}(\tau)\right\|_{D^{(0)}-\delta} & \leq \mu_{1}^{(0)}, \quad\left\|v^{(0)}(\tau)\right\|_{D^{(0)}-\delta} \leq \frac{\mu_{2}^{(0)}}{\delta+\delta^{(0)}}+1, \quad\left\|e^{(0)}(\tau)\right\|_{D^{(0)}-\delta} \leq \mu_{3}^{(0)}, \\
\left\|w^{(0)}(\tau)\right\|_{D^{(0)}-\delta} & \leq \mu_{4}^{(0)}\left(\frac{\log \left(\frac{1}{\delta+\delta^{(0)}}\right)}{\left(\delta+\delta^{(0)}\right)^{2}}+1\right), \quad\left\|f^{(0)}(\tau)\right\|_{D^{(0)}-\delta} \leq \mu_{9}^{(0)}, \\
0<\mu_{5}^{(0)} \leq\left|a^{(0)}\right| & \leq \mu_{6}^{(0)}, \quad\left\|V^{\prime \prime}(q)\right\|_{D^{(0)}+\mu_{1}^{(0)}+1} \leq \mu_{7}^{(0)}, \quad\left\|V^{\prime \prime \prime}(q)\right\|_{D^{(0)}+\mu_{1}^{(0)}+1} \leq \mu_{8}^{(0)},
\end{aligned}
$$

where we recall that $a^{(0)}$ has been introduced in such a way that $\left\langle\operatorname{det}\left(P^{(0)}\right)\right\rangle=1$ (see (56)). In fact, for this initial approximation, and using that $v^{(0)}(\tau)$ is an exact solution of the inner equation, one can check easily that $\operatorname{det}\left(P^{(0)}\right)(\tau)=1$, and that $a^{(0)}=1$ (to check this we only have to consider $\operatorname{Im}(\tau)$ going to $+\infty)$. We remark that the bounds for $l^{(0)}(\tau)$ and for the error function $f^{(0)}(\tau)$ are taken independent of $\delta$, despite the expressions given in (92) depends on $\delta$, in order to simplify the recursive bound in the proof. Then, assuming $0 \leq \varepsilon<1$, we have the following values for the expressions $\mu_{j}^{(0)}, j=1, \ldots, 9$ :

$$
\begin{aligned}
& \mu_{1}^{(0)}=M, \quad \mu_{2}^{(0)}=M, \quad \mu_{3}^{(0)}=M B \varepsilon^{2}, \quad \mu_{4}^{(0)}=M, \\
& \mu_{5}^{(0)}=1, \quad \mu_{6}^{(0)}=1, \quad \mu_{7}^{(0)}=\frac{1}{2}\left(R_{0}+B\right), \quad \mu_{8}^{(0)}=\frac{1}{2}\left(R_{0}+B\right), \\
& \mu_{9}^{(0)}=M B \varepsilon^{2}\left(\frac{\log \left(1 / \delta^{(0)}\right)}{\left(\delta^{(0)}\right)^{2}}+1\right)=\mu_{3}^{(0)}\left(\frac{\log \left(1 / \delta^{(0)}\right)}{\left(\delta^{(0)}\right)^{2}}+1\right) .
\end{aligned}
$$

Now, we apply the iterative method introduced in Section 4, obtaining the following quantitative result (see Section A.3 for the proof).

Theorem 3 We assume the notations introduced before in this section, and we put $\omega=$ $2 \pi \gamma$, where $\gamma$ is the golden mean. We consider the sequences $v^{(n)}(\tau), w^{(n)}(\tau)$ and $a^{(n)}$ defined recursively in Section 4, starting with $v^{(0)}(\tau), w^{(0)}(\tau)$ and $a^{(0)}=1$ as defined above. We take as initial domain $D^{(0)}=\mathcal{D}\left(-\log R_{0}+\delta^{(0)}, \log B\right)$, where $B$ is any constant verifying $B>\max \left\{e^{2} / R_{0}, 1\right\}$, and

$$
0<\delta^{(0)}<\min \left\{1, \frac{\log B+\log R_{0}-2}{21}\right\} .
$$

Then, there exists a constant $c \geq 1$, depending on $\mu_{1}^{(0)}, \mu_{2}^{(0)}, \mu_{4}^{(0)}, \mu_{5}^{(0)}, \mu_{6}^{(0)}, \mu_{7}^{(0)}$ and $\mu_{8}^{(0)}$, such that if $\mu_{3}^{(0)}$ is small enough in order to have,

$$
\Delta \equiv 12 c \mu_{3}^{(0)} \frac{\left(\log \left(\frac{1}{\delta^{(0)}}\right)\right)^{2}}{\left(\delta^{(0)}\right)^{8}} \leq \frac{1}{4},
$$

we can assure that these sequences converge to $v(\tau)=\tau+l(\tau), w(\tau)$ and a, with $v(\tau)$ and $w(\tau)$ analytic functions, defined at least in the complex strip $\widetilde{\mathbf{D}}=\mathcal{D}\left(-\log R_{0}+\right.$ $\left.11 \delta^{(0)}, \log B-10 \delta^{(0)}\right), 2 \pi$-periodic in $\tau$ and with $\langle l\rangle=0$. They verify that $v(\tau)$ is solution of the Lagrangian equation (85), and $z(\tau)=w(\tau)+\frac{a}{\omega} \tau v^{\prime}(\tau)$ is solution of the variationals of (85) around $v(\tau)$, with the constant a in $\mathbb{R}$ given in such a way $\operatorname{det}(P(\tau))=1$ (see (52)). 
Moreover, in this domain we have for $l(\tau), w(\tau)$ and a the bounds (92), now with $\mu_{j}=\mu_{j}^{(0)}+6 c \mu_{3}^{(0)} \log \left(1 / \delta^{(0)}\right) /\left(\delta^{(0)}\right)^{4}$, for $j=1,2, \mu_{4}=\mu_{4}^{(0)}+6 c \mu_{3}^{(0)}\left(\log \left(1 / \delta^{(0)}\right)\right)^{2} /\left(\delta^{(0)}\right)^{8}$, $\mu_{5}=\mu_{5}^{(0)} / 2$ and $\mu_{6}=2 \mu_{6}^{(0)}$, where $\mu_{j} \leq \mu_{j}^{(0)}+1$, for $j=1,2,4$.

On the other hand $l(\tau)$ and $w(\tau)$ are small perturbations of $l^{0}(\tau)$ and $w^{(0)}(\tau)$, in the sense that

$$
\begin{aligned}
&\left\|l(\tau)-l^{(0)}(\tau)\right\|_{\widetilde{\mathbf{D}}-\delta} \leq 6 c \mu_{3}^{(0)} \frac{\log \left(\frac{1}{\delta^{(0)}}\right)}{\left(\delta^{(0)}\right)^{4}} \\
&\left\|l^{\prime}(\tau)-l^{(0)}(\tau)\right\|_{\widetilde{\mathbf{D}}_{-} \delta} \leq \frac{6 c \mu_{3}^{(0)} \frac{\log \left(\frac{1}{\delta^{(0)}}\right)}{\left.\delta+\delta^{(0)}\right)^{4}}}{\delta+\delta^{(0)}} \\
&\left\|w(\tau)-w^{(0)}(\tau)\right\|_{\widetilde{\mathbf{D}}_{-\delta}} \leq 6 c \mu_{3}^{(0)} \frac{\left(\log \left(\frac{1}{\delta^{(0)}}\right)\right)^{2}}{\left(\delta^{(0)}\right)^{8}} \frac{\log \left(\frac{1}{\delta+\delta^{(0)}}\right)}{\left(\delta+\delta^{(0)}\right)^{2}} .
\end{aligned}
$$

Now, using the values of the constants $\mu_{j}^{(0)}$ given in (93), we can give a more quantitative result. Let us take $\delta^{(0)}=\varepsilon^{1 / 4-\alpha}$, for any value $0<\alpha<1 / 4$ (fixed from now on). With this choice, it is clear that (94) is achieved if $\varepsilon$ is small enough, and for (95) we have:

$$
\Delta=12 c M B(1 / 4-\alpha)^{2} \varepsilon^{8 \alpha}(\log (1 / \varepsilon))^{2}<1 / 4,
$$

provided that $\varepsilon$ is small enough. Then, we have that the solution $v(\tau)$ exists in a domain that is $\mathcal{O}\left(\varepsilon^{1 / 4-\alpha}\right)$ close to the initial one $\mathcal{D}\left(-\log R_{0}, \log B\right)$. More concretely, going back to the original variables given by (6) and (7), we obtain the following result:

Corollary 3 Let us take $\alpha$ any constant verifying $0<\alpha<1 / 4$. Then, if $\varepsilon$ is small enough to verify (96), the Lagrangian equation (5), for $V(q)=-\varepsilon \cos q$ and $\omega=2 \pi \gamma$, has a solution $\bar{u}(\theta)=\theta+\bar{\ell}(\theta)$, where $\bar{\ell}(\theta)$ is analytic, $2 \pi$-periodic in $\theta$ and with $\langle\bar{\ell}\rangle=0$. Moreover, $\bar{u}(\theta)$ is defined at least for $\theta \in \overline{\mathbf{D}}$, where

$$
\overline{\mathbf{D}}=\mathcal{D}\left(-\log (1 / \varepsilon)-\log R_{0}+11 \varepsilon^{1 / 4-\alpha},-\log (1 / \varepsilon)+\log B-10 \varepsilon^{1 / 4-\alpha}\right),
$$

Moreover, in this domain we have the estimate

$$
\|\bar{\ell}(\theta)-m(\theta+i \log (1 / \varepsilon)-\pi)\|_{\overline{\mathbf{D}}} \leq \varepsilon \sqrt{\frac{3 M c B}{4}}
$$

where $c \geq 1$ is the constant provided by Theorem 3 (that is an increasing function of $B$ ).

Proof: It is straightforward from Theorem 3 and the condition for $\varepsilon$ obtained in (96).

\subsection{Proof of Theorem 1: complex matching}

Now, we are in conditions to prove Theorem 1 . To do that, we only have to show that the solutions $u(\theta)$ (provided by Corollary 1) and $\bar{u}(\theta)$ (provided by Corollary 3 ) of equation (5), for $V(q)=-\varepsilon \cos q$ and $\omega=2 \pi \gamma$, are both defined in a suitable common domain, and that in this domain they are close enough. So, we only have to apply Proposition 1 to assure that $\bar{u}(\theta)$ is the analytic continuation of $u(\theta)$. The proof can be summarized in the following steps: 


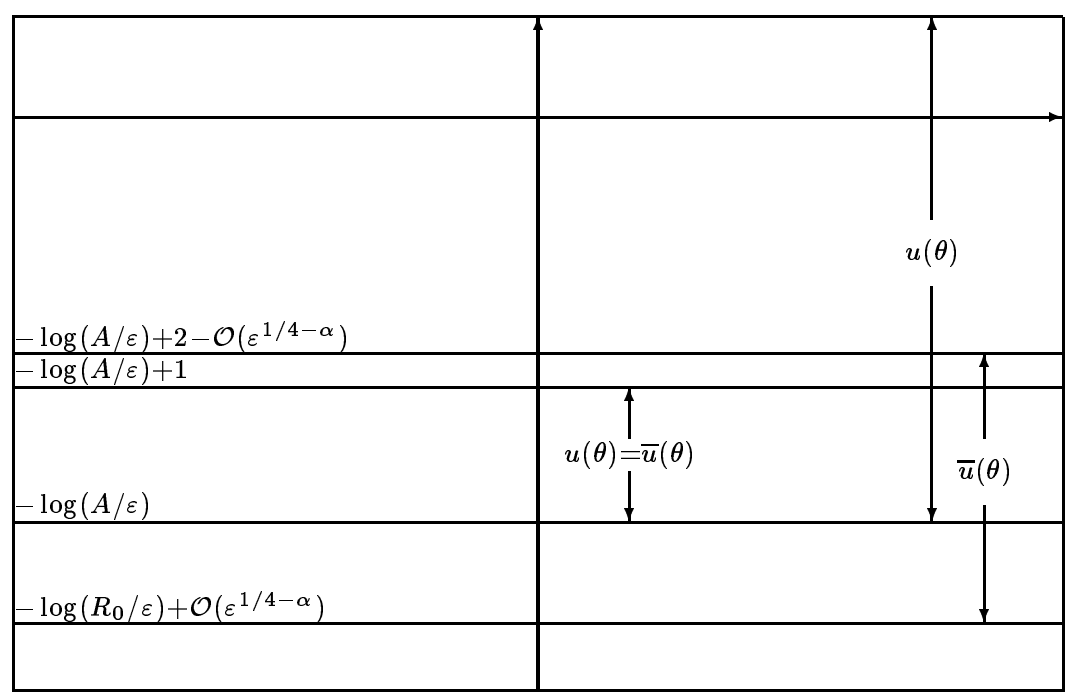

Figure 1: Domains of analyticity of $u(\theta)$ and $\bar{u}(\theta)$ and matching domain.

1. We consider a fixed value of $0<\alpha<1 / 4$.

2. Let us call $c$ the constant given by Corollary 1 , and let us consider the value $\bar{\Delta}=\frac{1}{16 c \mathrm{e}}$ provided by Proposition 1 , with $\sigma=2, \mu_{7}^{(0)}=\mathrm{e} / 2$ and $\bar{\rho}_{2}-\bar{\rho}_{1}=1$.

3. Let us take $A^{(0)}$ any constant verifying $A^{(0)}<\min \left\{\frac{1}{16 c}, \frac{3 \bar{\Delta}^{2}}{4 c}\right\}$. From Corollary 1 we have that, if $\varepsilon$ is small enough (depending on $\left.A^{(0)}\right)$, then $\|\ell(\theta)\|_{-\log (A / \varepsilon), \log (A / \varepsilon)} \leq$ $\bar{\Delta} / 2$, for any $0<A \leq A^{(0)}$.

4. We define $B_{0}=4 M / \bar{\Delta}$, where $M \geq 1$ is the constant introduced in (91). Thus, we have that if $B \geq B_{0}$, then $\|m(\tau)\|_{-\log R_{0}+\log B,+\infty} \leq \bar{\Delta} / 4$, and so, we have the same bound for $m(\theta+i \log (1 / \varepsilon)-\pi)$ in the domain $\mathcal{D}\left(-\log (1 / \varepsilon)-\log R_{0}+\log B,+\infty\right)$ (we recall that $l^{(0)}(\tau) \equiv m(\tau)$ in $(91)$ ).

5. We consider a fixed $B$ defined by means of the equality $\log B=-\log A+2$, where we take $0<A \leq A^{(0)}$ small enough, in such a way $B \geq \max \left\{B_{0}, \mathrm{e}^{2} / R_{0}, 1\right\}$. Provided with this value of $B$, we apply Theorem 3 , with the formulation stated in Corollary 3 , obtaining for the solution $\bar{\ell}(\theta)$ the estimate $\|\bar{\ell}(\theta)-m(\theta+i \log (1 / \varepsilon)-\pi)\|_{\overline{\mathbf{D}}} \leq \bar{\Delta} / 4$, if $\varepsilon$ is small enough, depending on $M, B$ and $c$ ( $c$ is now the value provided by Theorem 3). Let us point that $\mathcal{D}(-\log (1 / \varepsilon)-\log A,-\log (1 / \varepsilon)+\log B-1) \subset \overline{\mathbf{D}}$, at least for small values of $\varepsilon$ (we note that $A \leq R_{0}$ ).

6. So, we can do the complex matching applying Proposition 1 in the domain $\mathcal{D}\left(\bar{\rho}_{1}, \bar{\rho}_{2}\right)$, where $\bar{\rho}_{1}=-\log (A / \varepsilon)$ and $\bar{\rho}_{2}=-\log (A / \varepsilon)+1$, with $\bar{\Delta}$ as given in item 2 . because we have $\bar{\rho}_{2}-\bar{\rho}_{1}=1$. To do that, let us point that in this case $\mu_{1}^{(0)}=0$, and it is clear that $\|\bar{\ell}(\theta)\|_{-\log (A / \varepsilon),-\log (A / \varepsilon)+1} \leq \bar{\Delta} / 4+\|\bar{\ell}(\theta)-m(\theta+i \log (1 / \varepsilon)-\pi)\|_{\overline{\mathbf{D}}} \leq \bar{\Delta} / 2 \leq 1$. 
To obtain the analytic continuation of the function $u(\theta)$ in the upper plane, we only have to do an analogous construction, or to use the symmetries of the standard map, in order to obtain a symmetric domain with respect to the real axis.

Finally, the estimates provided by Corollary 3 give that $\bar{\ell}(\theta)$ (or $\ell(\theta)$, as we have proved that both are the same function), is asymptotic to $m(\theta+i \log (1 / \varepsilon)-\pi)$, as $\varepsilon \rightarrow 0$, at least in the domain $\overline{\mathbf{D}}$, as stated in the item $(c)$ of Theorem 1.

\section{A Proof of the convergence theorems}

In order to help in the readability of this paper, we have placed the proof of the most technical results in this last part of the work. So, in Appendix A we give the proof of Theorem 2 and Proposition 1 (see Section A.2), as well the proof of Theorem 3 (see Section A.3). Moreover, in Section A.4 we also have included the proof of some estimates on small divisors related to the golden mean, that have been used to prove Lemma 2 .

\section{A.1 Some basic properties of the norm}

First of all we recall some definitions we have introduced to work with $2 \pi$-periodic analytic functions. We have considered functions defined in complex domains of the form

$$
\mathcal{D}\left(\rho_{1}, \rho_{2}\right)=\left\{z \in \mathbb{C}: \rho_{1} \leq \operatorname{Im}(\theta) \leq \rho_{2}\right\}
$$

with $-\infty \leq \rho_{1} \leq \rho_{2} \leq+\infty$. Moreover, given an analytic function $f(\theta), 2 \pi$-periodic in $\theta$, and defined in this domain, we have introduced (from its Fourier expansion (23)) the norm

$$
\|f\|_{\rho_{1}, \rho_{2}}=\left|f_{0}\right|+\sum_{k>0}\left|f_{k}\right| \mathrm{e}^{-k \rho_{1}}+\sum_{k<0}\left|f_{k}\right| \mathrm{e}^{-k \rho_{2}} .
$$

As we have mentioned before, this definition keeps several properties of the supremum norm. In the present section we are going to display some of these properties, whose proof can be checked using the definition of this norm and the basic properties of Fourier series.

Lemma 4 Let us take $F(\theta), g(\theta)$ and $h(\theta)$ analytic functions, $2 \pi$-periodic in $\theta$, and with $\|g\|_{\rho_{1}, \rho_{2}} \leq \delta$ and $\|h\|_{\rho_{1}, \rho_{2}} \leq \delta$. Then, if all the estimates on $F$ involved in the following expressions are well defined, we have:

- $\|\cdot\|_{\rho_{1}, \rho_{2}}$ is a multiplicative norm:

$$
\|g(\theta) h(\theta)\|_{\rho_{1}, \rho_{2}} \leq\|g(\theta)\|_{\rho_{1}, \rho_{2}}\|h(\theta)\|_{\rho_{1}, \rho_{2}} .
$$

- It is preserved by the composition:

$$
\|F(\theta+g(\theta))\|_{\rho_{1}, \rho_{2}} \leq\|F(\theta)\|_{\rho_{1}-\delta, \rho_{2}+\delta} .
$$

- We have the following "mean value theorem":

$$
\|F(\theta+g(\theta))-F(\theta+h(\theta))\|_{\rho_{1}, \rho_{2}} \leq\left\|F^{\prime}(\theta)\right\|_{\rho_{1}-\delta, \rho_{2}+\delta}\|g(\theta)-h(\theta)\|_{\rho_{1}, \rho_{2}} .
$$


- The standard bounds for the remainder of the Taylor formula up to order 1 are also true:

$$
\begin{aligned}
& \left\|F(\theta+g(\theta))-F(\theta+h(\theta))-F^{\prime}(\theta+h(\theta))(g(\theta)-h(\theta))\right\|_{\rho_{1}, \rho_{2}} \\
& \leq \frac{1}{2}\left\|F^{\prime \prime}(\theta)\right\|_{\rho_{1}-\delta, \rho_{2}+\delta}\|g(\theta)-h(\theta)\|_{\rho_{1}, \rho_{2}}^{2} .
\end{aligned}
$$

- We have the following Cauchy-like estimates for the derivative:

$$
\left\|F^{\prime}(\theta)\right\|_{\rho_{1}+\delta, \rho_{2}-\delta} \leq \frac{\|\widetilde{F}(\theta)\|_{\rho_{1}, \rho_{2}}}{\mathrm{e} \delta}
$$

where we recall that $\tilde{F}(\theta)$ is defined in (22).

- If $\langle F\rangle=0$, then we have

$$
\left\|\mathcal{L}_{\omega}^{-1}(F)\right\|_{\rho_{1}+\delta, \rho_{2}-\delta} \leq \frac{1}{4 c_{\omega}}\left(\frac{\sigma-1}{\mathrm{e} \delta}\right)^{\sigma-1}\|F\|_{\rho_{1}, \rho_{2}}
$$

where the operator $\mathcal{L}_{\omega}^{-1}$ is defined in (24), provided that $\omega$ verifies the Diophantine condition (4). The proof follows from some estimates on the small divisors that have been also used to prove Lemma 2.

\section{A.2 Proof of Theorem 2 and Proposition 1}

In this section we are going to prove that, under the conditions displayed in Theorem 2, the sequence $u^{(n)}(\theta)$ constructed in Section 2.3 converges to a solution of equation (5). Moreover we will also prove Proposition 1, that gives conditions to ensure the uniqueness of this solution.

\section{A.2.1 Iterative lemma}

First of all, we study the effect of one step of the Newton method on a given approximation.

Lemma 5 We consider a fixed $\omega \in \mathbb{R}$ of Diophantine type (4), for certain $\sigma \geq 2$ and $c_{\omega}>0$, and $V(q)$ an entire function, $2 \pi$-periodic in $q$. Let $\ell^{(n)}(\theta)$ be an analytic function defined on $\mathcal{D}\left(\rho_{1}^{(n)}, \rho_{2}^{(n)}\right)$, for certain $-\infty \leq \rho_{1}^{(n)}<\rho_{2}^{(n)} \leq+\infty, 2 \pi$-periodic in $\theta$ and with $\left\langle\ell^{(n)}\right\rangle=0$. We put $u^{(n)}(\theta)=\theta+\ell^{(n)}(\theta)$, and we assume the following bounds:

$$
\begin{aligned}
& \left\|\ell^{(n)}(\theta)\right\|_{\rho_{1}^{(n)}, \rho_{2}^{(n)}} \leq \mu_{1}^{(n)}, \quad\left\|\ell^{\prime(n)}(\theta)\right\|_{\rho_{1}^{(n)}, \rho_{2}^{(n)}} \leq \mu_{2}^{(n)}, \quad\left\|e^{(n)}(\theta)\right\|_{\rho_{1}^{(n)}, \rho_{2}^{(n)}} \leq \mu_{3}^{(n)}, \\
& \left\|\frac{1}{u^{\prime(n)}(\theta)}\right\|_{\rho_{1}^{(n)}, \rho_{2}^{(n)}} \leq \mu_{4}^{(n)}, \quad 0<\mu_{5}^{(n)} \leq\left|a^{(n)}\right| \leq \mu_{6}^{(n)}, \quad\left\|V^{\prime \prime \prime}(q)\right\|_{\rho_{1}^{(n)}-\mu_{1}^{(0)}-1, \rho_{2}^{(n)}+\mu_{1}^{(0)}+1} \leq \mu_{7}^{(n)},
\end{aligned}
$$

with $\mu_{1}^{(n)}<\mu_{1}^{(0)}+1, \mu_{2}^{(n)}<\mu_{2}^{(0)}+1$, for certain $\mu_{1}^{(0)} \geq 0$ and $\mu_{2}^{(0)} \geq 0$, and $\mu_{3}^{(n)} \leq 1$, where $e^{(n)}(\theta)$ is defined in (11) and $a^{(n)}$ is given in (20). Then, there exist $c^{(n)} \geq 1, c^{(n)} \equiv$ $c\left(\mu_{1}^{(0)}, \mu_{2}^{(0)}, \mu_{4}^{(n)}, 1 / \mu_{5}^{(n)}, \mu_{6}^{(n)}, \mu_{7}^{(n)}\right)$, that it is an increasing function of all its arguments, such that given any $\delta^{(n)}$, with

$$
0<\delta^{(n)} \leq \min \left\{1, \frac{\rho_{2}^{(n)}-\rho_{1}^{(n)}}{6}\right\}
$$


for which

$$
c^{(n)} \frac{\mu_{3}^{(n)}}{\left(\delta^{(n)}\right)^{2 \sigma-1}} \leq \min \left\{\frac{1}{2}, 1+\mu_{2}^{(0)}-\mu_{2}^{(n)}\right\}, \quad c^{(n)} \frac{\mu_{3}^{(n)}}{\left(\delta^{(n)}\right)^{2(\sigma-1)}} \leq 1+\mu_{1}^{(0)}-\mu_{1}^{(n)}
$$

then we can apply a step of the Newton method formulated in Section 2.2 to compute a new iterate $u^{(n+1)}(\theta)=\theta+\ell^{(n+1)}(\theta)$, with $\left\langle\ell^{(n+1)}\right\rangle=0$, verifying

$$
\begin{aligned}
\left\|\ell^{(n+1)}-\ell^{(n)}\right\|_{\rho_{1}^{(n+1)}, \rho_{2}^{(n+1)}} & \leq c^{(n)} \frac{\mu_{3}^{(n)}}{\left(\delta^{(n)}\right)^{2(\sigma-1)}}, \\
\left\|\ell^{(n+1)}-\ell^{\prime(n)}\right\|_{\rho_{1}^{(n+1)}, \rho_{2}^{(n+1)}} & \leq c^{(n)} \frac{\mu_{3}^{(n)}}{\left(\delta^{(n)}\right)^{2 \sigma-1}}, \\
\left\|e^{(n+1)}\right\|_{\rho_{1}^{(n+1)}, \rho_{2}^{(n+1)}} & \leq c^{(n)} \frac{\left(\mu_{3}^{(n)}\right)^{2}}{\left(\delta^{(n)}\right)^{4(\sigma-1)}}, \\
\| \frac{1}{u^{(n+1)} \|_{\rho_{1}^{(n+1)}, \rho_{2}^{(n+1)}}} & \leq \frac{\mu_{4}^{(n)}}{1-c^{(n)} \frac{\mu_{3}^{(n)}}{\left(\delta^{(n)}\right)^{2 \sigma-1}}}, \\
\left|a^{(n+1)}-a^{(n)}\right| & \leq c^{(n)} \frac{\mu_{3}^{(n)}}{\left(\rho_{2}^{(n)}-\rho_{1}^{(n)}\right)^{2 \sigma-1}}
\end{aligned}
$$

where $\rho_{1}^{(n+1)}=\rho_{1}^{(n)}+3 \delta^{(n)}$ and $\rho_{2}^{(n+1)}=\rho_{2}^{(n)}-3 \delta^{(n)}$.

Proof: In order to simplify the notation, we will skip the super-script $(n)$ everywhere along the proof, and we will use the super-script $(1)$ to label the $(n+1)$ iteration. First of all, let us explain the role played by the constant $c$ on the statement. We are going to use always the same notation, $c$, for the different bounds appearing in the proof, that are function of the arguments in which $c$ depends. So, this constant has to be re-defined in a recurrent way along the proof of this lemma, in order to meet all the bounds where it is involved (a finite number of them), and the value of $c$ obtained after the last bound is the one claimed in the statement. Moreover, and except when it is explicitly mentioned, during the proof we will avoid the restriction $\delta<1$. Then, the remaining bounds hold for any value of $\delta$ for which the expressions involved are well defined.

In the following estimates we will use the properties of the weighted-norm given in Lemma 4. First of all, going back to (20) and (21), we have

$$
\|h(\theta)\|_{\rho_{1}, \rho_{2}} \leq c, \quad\|\widetilde{h}(\theta)\|_{\rho_{1}, \rho_{2}} \leq c, \quad\|\nu(\theta)\|_{\rho_{1}+\delta, \rho_{2}-\delta} \leq \frac{c}{\delta^{\sigma-1}}
$$

These bounds give

$$
\left\|u^{\prime}(\theta) e(\theta)\right\|_{\rho_{1}, \rho_{2}} \leq c \mu_{3}, \quad\left\|\nu(\theta) u^{\prime}(\theta) e(\theta)\right\|_{\rho_{1}+\delta, \rho_{2}-\delta} \leq c \frac{\mu_{3}}{\delta^{\sigma-1}}, \quad\left|\left\langle\nu u^{\prime} e\right\rangle\right| \leq c \frac{\mu_{3}}{\left(\rho_{2}-\rho_{1}\right)^{\sigma-1}}
$$

Let us point that when bounding the average by the norm, we use the Cauchy estimates given in Lemma 4 with the maximum reduction allowed in the width of the strip of analyticity, which in this case is $\delta=\left(\rho_{2}-\rho_{1}\right) / 2$. Now, we solve (29), with the conditions (31) and (32), and we compute $\xi^{(n)}(\theta)$ from (28). Then, using the same kind of arguments, 
one obtains

$$
\begin{aligned}
\left\|\tilde{\eta}_{2}(\theta)\right\|_{\rho_{1}+\delta, \rho_{2}-\delta} & \leq c \frac{\mu_{3}}{\delta^{\sigma-1}}, \quad\left|\left\langle\eta_{2}\right\rangle\right| \leq c \frac{\mu_{3}}{\left(\rho_{2}-\rho_{1}\right)^{\sigma-1}}, \\
\left\|\eta_{2}(\theta)\right\|_{\rho_{1}+\delta, \rho_{2}-\delta} & \leq c \frac{\mu_{3}}{\delta^{\sigma-1}}, \quad\left\|\widetilde{\eta}_{1}(\theta)\right\|_{\rho_{1}+2 \delta, \rho_{2}-2 \delta} \leq c \frac{\mu_{3}}{\delta^{2(\sigma-1)}} \\
\left|\left\langle\eta_{1}\right\rangle\right| & \leq c \frac{\mu_{3}}{\left(\rho_{2}-\rho_{1}\right)^{2(\sigma-1)}}, \quad\left\|\eta_{1}(\theta)\right\|_{\rho_{1}+2 \delta, \rho_{2}-2 \delta} \leq c \frac{\mu_{3}}{\delta^{2(\sigma-1)}} \\
\|\xi(\theta)\|_{\rho_{1}+2 \delta, \rho_{2}-2 \delta} & \leq c \frac{\mu_{3}}{\delta^{2(\sigma-1)}}, \quad\left\|\xi^{\prime}(\theta)\right\|_{\rho_{1}+3 \delta, \rho_{2}-3 \delta} \leq c \frac{\mu_{3}}{\delta^{2 \sigma-1}} .
\end{aligned}
$$

Thus, the bounds (99) for $\left\|\ell^{(1)}(\theta)-\ell(\theta)\right\|$ and $\left\|\ell^{\prime(1)}(\theta)-\ell^{\prime}(\theta)\right\|$ are clear. To bound the new error $e^{(1)}(\theta)$, we recall the decomposition

$$
e^{(1)}(\theta)=e_{1}^{(1)}(\theta)+e_{2}^{(1)}(\theta)
$$

that we have done in (33) and (34). To estimate $e_{2}^{(1)}(\theta)$, we remark that condition (98) guarantee that $\left\|\ell^{(1)}\right\|_{\rho_{1}+2 \delta, \rho_{2}-2 \delta} \leq \mu_{1}^{(0)}+1$. Then, we can use Lemma 4 to bound the remainder of the Taylor formula up to order 1 in the domain $\mathcal{D}\left(\rho_{1}-\mu_{1}^{(0)}-1, \rho_{2}+\mu_{1}^{(0)}+1\right)$. On the other hand, $e_{1}^{(1)}(\theta)$ can be bounded looking at its equivalent expression (36), having

$$
\left\|e_{1}^{(1)}\right\|_{\rho_{1}^{(1)}, \rho_{2}^{(1)}} \leq c \frac{\mu_{3}^{2}}{\delta^{2 \sigma-1}}, \quad\left\|e_{2}^{(1)}\right\|_{\rho_{1}^{(1)}, \rho_{2}^{(1)}} \leq c \frac{\mu_{3}^{2}}{\delta^{4(\sigma-1)}} .
$$

Recalling that to display the results we will take $0<\delta \leq 1$, the worst bound is the one given by $e_{2}^{(1)}(\theta)$, and it is the one taken into account in (99).

The next step is to consider $1 / u^{\prime(1)}(\theta)$. For this purpose, we write

$$
\frac{1}{u^{\prime(1)}(\theta)}=\frac{1}{u^{\prime}(\theta)+\xi^{\prime}(\theta)}=\frac{1}{u^{\prime}(\theta)}\left(\frac{1}{1+\frac{\xi^{\prime}(\theta)}{u^{\prime}(\theta)}}\right),
$$

and from here, we derive the estimate

$$
\left\|\frac{1}{u^{\prime(1)}(\theta)}\right\|_{\rho_{1}+3 \delta, \rho_{2}-3 \delta} \leq \frac{\mu_{4}}{1-c \frac{\mu_{3}}{\delta^{2 \sigma-1}}}
$$

where we use that $c \mu_{3} / \delta^{2 \sigma-1} \leq 1 / 2$ (see (98)). Finally, to end this proof we only have to estimate $a^{(1)}-a$, or in equivalent way, $\left\langle h^{(1)}-h\right\rangle$. Then, we notice that

$$
h^{(1)}(\theta)-h(\theta)=\frac{u^{\prime}(\theta) u^{\prime}(\theta+\omega)-u^{\prime(1)}(\theta) u^{\prime(1)}(\theta+\omega)}{u^{\prime}(\theta) u^{\prime}(\theta+\omega) u^{\prime(1)}(\theta) u^{\prime(1)}(\theta+\omega)} .
$$

To bound this expression, we add and subtract $u^{\prime}(\theta) u^{\prime(1)}(\theta+\omega)$ at the numerator of this fraction. Now, using that $\left\|\ell^{\prime(1)}\right\|_{\rho_{1}+3 \delta, \rho_{2}-3 \delta} \leq 1+\mu_{2}^{(0)}$, which is also guaranteed by the proved bounds (99) and the hypotheses (98), we have

$$
\left|\left\langle h^{(1)}-h\right\rangle\right| \leq c \frac{\mu_{3}}{\left(\rho_{2}-\rho_{1}\right)^{2 \sigma-1}} .
$$

To obtain this estimate, we point that have taken again the maximum reduction allowed in the width of the strip of analyticity, in order to bound $\left|\left\langle\xi^{\prime}\right\rangle\right|$ by $\left\|\xi^{\prime}\right\|$. 


\section{A.2.2 Proof of the convergence}

The proof of Theorem 2 relays on the convergence of the sequence $u^{(n)}(\theta)$, that will be proved by induction using the iterative Lemma 5 .

We put $\delta^{(n)}=\delta^{(0)} / 2^{n}$, and we have $\sum_{n=0}^{\infty} \delta^{(n)}=2 \delta^{(0)}$. Then, we want to apply iteratively Lemma 5 , starting with $u^{(0)}(\theta)$ in $\mathcal{D}\left(\rho_{1}^{(0)}, \rho_{2}^{(0)}\right)$, and using at any step $\delta \equiv \delta^{(n)}$. Hence, it is clear that $\rho_{1}^{(n)}<\rho_{1}^{(0)}+6 \delta^{(0)}$ and $\rho_{2}^{(n)}>\rho_{2}^{(0)}-6 \delta^{(0)}$.

Let us proceed by induction. We assume done $n$ steps of this iterative method, and that for $u^{(n)}(\theta)$ we have that the constants $\mu_{j}^{(n)}, j=1, \ldots, 7$, defined as in (97), verify $\mu_{1}^{(n)} \leq \mu_{1}^{(0)}+1, \mu_{2}^{(n)} \leq \mu_{2}^{(0)}+1, \mu_{3}^{(n)} \leq 1, \mu_{4}^{(n)} \leq \mathrm{e} \mu_{4}^{(0)}, 1 / \mu_{5}^{(n)} \leq 2 / \mu_{5}^{(0)}, \mu_{6}^{(n)} \leq 2 \mu_{6}^{(0)}$. Moreover, it is clear that $\mu_{7}^{(n)} \leq \mu_{7}^{(0)}$. We also assume that

$$
c \frac{\mu_{3}^{(n)}}{\left(\delta^{(n)}\right)^{2 \sigma-1}} \leq \min \left\{\frac{1}{2}, 1+\mu_{2}^{(0)}-\mu_{2}^{(n)}\right\}, \quad c \frac{\mu_{3}^{(n)}}{\left(\delta^{(n)}\right)^{2(\sigma-1)}} \leq 1+\mu_{1}^{(0)}-\mu_{1}^{(n)},
$$

where $c$ is the function provided by the iterative lemma valued in:

$$
c=c\left(\mu_{1}^{(0)}+1, \mu_{2}^{(0)}+1, \mathrm{e} \mu_{4}^{(0)}, 2 / \mu_{5}^{(0)}, 2 \mu_{6}^{(0)}, \mu_{7}^{(0)}\right) .
$$

Let us note that this choice of $c$ implies that $c^{(j)} \leq c$, if $j \leq n$, and then conditions (101) guarantee that Lemma 5 can be applied, giving the following constants for the $n+1$ step:

$$
\begin{aligned}
& \mu_{1}^{(n+1)}=\mu_{1}^{(n)}+c \frac{\mu_{3}^{(n)}}{\left(\delta^{(n)}\right)^{2(\sigma-1)}}, \quad \mu_{2}^{(n+1)}=\mu_{2}^{(n)}+c \frac{\mu_{3}^{(n)}}{\left(\delta^{(n)}\right)^{2 \sigma-1}}, \\
& \mu_{3}^{(n+1)}=c \frac{\left(\mu_{3}^{(n)}\right)^{2}}{\left(\delta^{(n)}\right)^{4(\sigma-1)}}, \quad \mu_{4}^{(n+1)}=\frac{\mu_{4}^{(n)}}{1-c \frac{\mu_{3}^{(n)}}{\left(\delta^{(n)}\right)^{2 \sigma-1}}}, \\
& \mu_{5}^{(n+1)}=\mu_{5}^{(n)}-c \frac{\mu_{3}^{(n)}}{\left(\rho_{2}^{(n)}-\rho_{1}^{(n)}\right)^{2 \sigma-1}}, \quad \mu_{6}^{(n+1)}=\mu_{6}^{(n)}+c \frac{\mu_{3}^{(n)}}{\left(\rho_{2}^{(n)}-\rho_{1}^{(n)}\right)^{2 \sigma-1}} .
\end{aligned}
$$

Thus, the induction implies

$$
\mu_{1}^{(n+1)}=\mu_{1}^{(0)}+\sum_{j=0}^{n} c \frac{\mu_{3}^{(j)}}{\left(\delta^{(j)}\right)^{2(\sigma-1)}}, \quad \mu_{2}^{(n+1)}=\mu_{2}^{(0)}+\sum_{j=0}^{n} c \frac{\mu_{3}^{(j)}}{\left(\delta^{(j)}\right)^{2 \sigma-1}},
$$

and hence, the key point is to control $\mu_{3}^{(j)}$, that, from the definition of $\delta^{(j)}$, verifies

$$
\mu_{3}^{(j+1)}=2^{4 j(\sigma-1)} c \frac{\left(\mu_{3}^{(j)}\right)^{2}}{\left(\delta^{(0)}\right)^{4(\sigma-1)}},
$$

for any $j=0, \ldots, n$. This expression, and the definition of $\Delta$ given in (41), imply that

$$
\mu_{3}^{(j)} \leq\left(2^{\sigma-1}\right)^{2^{j+2}-4(j+1)}\left(\frac{c}{\left(\delta^{(0)}\right)^{4(\sigma-1)}}\right)^{2^{j}-1}\left(\mu_{3}^{(0)}\right)^{2^{j}}=\frac{2^{4(\sigma-1)}}{2^{4(j+1)(\sigma-1)}} \Delta^{2^{j}-1} \mu_{3}^{(0)},
$$

for $j=0, \ldots, n+1$. As we ask that $\Delta<1$ in (41), then we can ensure that the bound of $\mu_{3}^{(j)}$ it is strictly decreasing to zero with $j$. Consequently, the sums defining $\mu_{1}^{(n+1)}$ and 
$\mu_{2}^{(n+1)}$ can be bounded by geometric ones of ratios $\Delta / 2^{2(\sigma-1)}$ and $\Delta / 2^{2 \sigma-3}$, respectively, and both are smaller than $1 / 2$. Hence, we derive the estimates

$$
\begin{aligned}
& \mu_{1}^{(n+1)} \leq \mu_{1}^{(0)}+2 c \frac{\mu_{3}^{(0)}}{\left(\delta^{(0)}\right)^{2(\sigma-1)}}=\mu_{1}^{(0)}+2 \Delta \frac{\left(\delta^{(0)}\right)^{2(\sigma-1)}}{2^{4(\sigma-1)}} \\
& \mu_{2}^{(n+1)} \leq \mu_{2}^{(0)}+2 c \frac{\mu_{3}^{(0)}}{\left(\delta^{(0)}\right)^{2 \sigma-1}}=\mu_{2}^{(0)}+2 \Delta \frac{\left(\delta^{(0)}\right)^{2 \sigma-3}}{2^{4(\sigma-1)}}
\end{aligned}
$$

which, as $\delta^{(0)}<1$, are bounded respectively by $1+\mu_{1}^{(0)}$ and $1+\mu_{2}^{(0)}$. Let us observe that the arguments presented also guarantee inequalities (101) for $n+1$. Now we consider $\mu_{4}^{(n+1)}$, that verifies

$$
\mu_{4}^{(n+1)}=\left(\prod_{j=0}^{n} \frac{1}{1-c \frac{\mu_{3}^{(j)}}{\left(\delta^{(j)}\right)^{2 \sigma-1}}}\right) \mu_{4}^{(0)}
$$

where we recall that we have $c \mu_{3}^{(j)} /\left(\delta^{(j)}\right)^{2 \sigma-1} \leq 1 / 2, j=0, \ldots, n$, by hypothesis (101). If we take $\log (\cdot)$ of this product, and we use that

$$
-\log (1-x) \leq 2 x, \quad \text { if } \quad 0 \leq x \leq \frac{1}{2},
$$

we obtain

$$
\mu_{4}^{(n+1)} \leq \exp \left(2 \sum_{j=0}^{n} c \frac{\mu_{3}^{(j)}}{\left(\delta^{(j)}\right)^{2 \sigma-1}}\right) \mu_{4}^{(0)} \leq \mathrm{e} \mu_{4}^{(0)} .
$$

To control $\mu_{5}^{(n+1)}$ and $\mu_{6}^{(n+1)}$, we remark that $\rho_{2}^{(n)}-\rho_{1}^{(n)} \geq\left(\rho_{2}^{(0)}-\rho_{1}^{(0)}\right) / 2$, and hence, we only have to bound $\sum_{j=0}^{n} \mu_{3}^{(j)}$ by a geometric sum of ratio $\Delta / 2^{4(\sigma-1)} \leq 1 / 2$, obtaining

$$
\mu_{5}^{(n+1)} \geq \mu_{5}^{(0)}-2 c \mu_{3}^{(0)}\left(\frac{2}{\rho_{2}^{(0)}-\rho_{1}^{(0)}}\right)^{2 \sigma-1}, \mu_{6}^{(n+1)} \leq \mu_{6}^{(0)}+2 c \mu_{3}^{(0)}\left(\frac{2}{\rho_{2}^{(0)}-\rho_{1}^{(0)}}\right)^{2 \sigma-1} .
$$

Using (41) we have that $\mu_{5}^{(n+1)} \geq \mu_{5}^{(0)} / 2$ and $\mu_{6}^{(n+1)} \leq 2 \mu_{6}^{(0)}$, and then all the inductive hypotheses also hold for $n+1$. Hence, we can take $n$ going to infinity, which ensures the convergence of the process in the domain $\mathcal{D}\left(\rho_{1}^{(0)}+6 \delta^{(0)}, \rho_{2}^{(0)}-6 \delta^{(0)}\right)$. Moreover, the different bounds obtained along the proof also give the ones claimed in the statement of the theorem for the limit function $u(\theta)=\theta+\ell(\theta)$ in the final domain.

\section{A.2.3 Proof of Proposition 1}

In this section we are going to prove Proposition 1 to establish the (local) uniqueness of the solution of (5).

Let be $\bar{u}(\theta)=\theta+\bar{\ell}(\theta)$ another (analytic) solution of (5), defined in $\mathcal{D}\left(\bar{\rho}_{1}, \bar{\rho}_{2}\right)$, a domain which is contained in the domain of $u(\theta)$ provided by Theorem 2 , that is, $\mathcal{D}\left(\rho_{1}^{(0)}+\right.$ $\left.6 \delta^{(0)}, \rho_{2}^{(0)}-6 \delta^{(0)}\right), 2 \pi$-periodic in $\theta$ and with $\langle\bar{\ell}\rangle=0$. We want to check that if $\| \bar{\ell}(\theta)-$ $\ell(\theta) \|_{\bar{\rho}_{1}, \bar{\rho}_{2}}$ is small enough, in the sense that they verify $(42)$, then $u(\theta)$ and $\bar{u}(\theta)$ have to coincide.

For this purpose we define $U(\theta)=\bar{u}(\theta)-u(\theta)$, then, using that both functions are solution of (5), we can write

$$
\mathcal{L}_{\omega}^{2} U(\theta)=V^{\prime}(\bar{u}(\theta))-V^{\prime}(u(\theta))=V^{\prime \prime}(u(\theta)) U(\theta)-\bar{e}(\theta),
$$


where

$$
\bar{e}(\theta)=V^{\prime}(u(\theta))-V^{\prime}(\bar{u}(\theta))+V^{\prime \prime}(u(\theta)) U(\theta) .
$$

Now, we can use the explicit reducibility of the invariant curve $u(\theta)$ (see Section 2.1), to solve (102) for $U(\theta)$, and to express it as function of $\bar{e}(\theta)$. More concretely, if we write

$$
U(\theta)=u^{\prime}(\theta) \eta_{1}(\theta)+\nu(\theta) u^{\prime}(\theta) \eta_{2}(\theta),
$$

we have that $\eta(\theta)=\left(\eta_{1}(\theta), \eta_{2}(\theta)\right)$ is obtained as the $2 \pi$-periodic solution of the equations:

$$
\mathcal{L}_{\omega} \eta(\theta)=\left(\begin{array}{ll}
0 & a \\
0 & 0
\end{array}\right) \eta(\theta)+\left(\begin{array}{c}
\nu(\theta+\omega) \\
-1
\end{array}\right) u^{\prime}(\theta+\omega) \bar{e}(\theta+\omega),
$$

(see (29)) where $a$ and $\nu(\theta)$ are defined from $u(\theta)$ (see (20) and (21)). We recall that the only indetermination in this equation is the choice of $\left\langle\eta_{1}\right\rangle$, which have to be defined in such a way $U(\theta)$ has zero average. But let us also recall that to guarantee the well defined character of this construction, we need that $\left\langle u^{\prime} \bar{e}\right\rangle=0$. To check this, we notice that as $u(\theta)$ and $\bar{u}(\theta)$ verify $(5)$, we can write:

$$
\begin{aligned}
u^{\prime}(\theta) \bar{e}(\theta)= & V^{\prime}(u(\theta)) u^{\prime}(\theta)-\mathcal{L}_{\omega}^{2} \bar{\ell}(\theta)-\ell^{\prime}(\theta) \mathcal{L}_{\omega}^{2} \bar{\ell}(\theta)+(\bar{\ell}(\theta)-\ell(\theta)) \mathcal{L}_{\omega}^{2} \ell^{\prime}(\theta) \\
= & V^{\prime}(u(\theta)) u^{\prime}(\theta)-\mathcal{L}_{\omega}^{2} \bar{\ell}(\theta)+\left[\ell^{\prime}(\theta+\omega) \bar{\ell}(\theta)-\ell^{\prime}(\theta) \bar{\ell}(\theta-\omega)\right]+\left[2 \ell^{\prime}(\theta) \ell(\theta)\right] \\
& +\left[\ell^{\prime}(\theta-\omega) \bar{\ell}(\theta)-\ell^{\prime}(\theta) \bar{\ell}(\theta+\omega)\right]-\left[\left(\ell^{\prime}(\theta+\omega)+\ell^{\prime}(\theta-\omega)\right) \ell(\theta)\right],
\end{aligned}
$$

and from here is not difficult to check that $\left\langle u^{\prime} \bar{e}\right\rangle=0$.

It is clear from (103) that if $\bar{e}(\theta)$ is zero, so is $U(\theta)$. In principle, we only have, from the definition of $\bar{e}(\theta)$, that $\bar{e} \equiv \mathcal{O}\left(U^{2}\right)$, but from the hypothesis (42), it can be quantified by

$$
\|\bar{e}\|_{\bar{\rho}_{1}^{(0)}, \bar{\rho}_{2}^{(0)}} \leq \frac{1}{2} \mu_{7}^{(0)}\|U\|_{\bar{\rho}_{1}^{(0)}, \bar{\rho}_{2}^{(0)}}^{2} \equiv \bar{\mu}_{3}^{(0)},
$$

where we define $\bar{\rho}_{1}^{(0)}=\bar{\rho}_{1}$ and $\bar{\rho}_{2}^{(0)}=\bar{\rho}_{2}$, and where we have used Lemma 4 , and the definition of $\mu_{7}^{(0)}$ (see (39)). Now, let $c \geq 1$ be the constant provided by Lemma 5 , corresponding to $\mu_{1}^{(0)}, \mu_{2}^{(0)}, \mu_{4}^{(0)}, \mu_{5}^{(0)}, \mu_{6}^{(0)}$ and $\mu_{7}^{(0)}$, that is the same that appear in the statement of Theorem 2. Then, using that the definition of $U(\theta)$ is done in the same way than $\xi(\theta)$ in $(28)$, we obtain for $U(\theta)$ the same bound than for $\xi(\theta)$ in (100), giving

$$
\|U\|_{\bar{\rho}_{1}^{(1)}, \bar{\rho}_{2}^{(1)}} \leq c \frac{\bar{\mu}_{3}^{(0)}}{\left(\bar{\delta}^{(0)}\right)^{2(\sigma-1)}}
$$

where we take $\bar{\delta}^{(0)}=\left(\bar{\rho}_{2}^{(0)}-\bar{\rho}_{1}^{(0)}\right) / 4$, with $\bar{\rho}_{1}^{(1)}=\bar{\rho}_{1}^{(0)}+2 \bar{\delta}^{(0)}$ and $\bar{\rho}_{1}^{(2)}=\bar{\rho}_{1}^{(0)}-2 \bar{\delta}^{(0)}$. Hence, we have the new estimate

$$
\|\bar{e}\|_{\bar{\rho}_{1}^{(1)}, \bar{\rho}_{2}^{(1)}} \leq \frac{1}{2} \mu_{7}^{(0)}\|U\|_{\bar{\rho}_{1}^{(1)}, \bar{\rho}_{2}^{(1)}}^{2} \leq \frac{1}{2} \mu_{7}^{(0)}\left(c \frac{\bar{\mu}_{3}^{(0)}}{\left(\bar{\delta}^{(0)}\right)^{2(\sigma-1)}}\right)^{2} \equiv \bar{\mu}_{3}^{(1)} .
$$

And now on, we can reiterate the same construction iteratively by taking, for instance, $\bar{\delta}^{(n)}=\bar{\delta}^{(0)} / 2^{n}$, obtaining that $\|\bar{e}\|_{\bar{\rho}_{1}^{(n)}, \bar{\rho}_{2}^{(n)}} \leq \bar{\mu}_{3}^{(n)}, n \geq 0$, with

$$
\bar{\mu}_{3}^{(n+1)}=\frac{1}{2} \mu_{7}^{(0)}\left(c \frac{2^{2 n(\sigma-1)} \bar{\mu}_{3}^{(n)}}{\left(\bar{\delta}^{(0)}\right)^{2(\sigma-1)}}\right)^{2}, \quad n \geq 0 .
$$


From here, we derive that

$$
\begin{aligned}
\bar{\mu}_{3}^{(n)} & =\left(c \frac{\mu_{7}^{(0)} 2^{2(\sigma-1)}\|U\|_{\bar{\rho}_{1}^{(0)}, \bar{\rho}_{2}^{(0)}}}{2\left(\bar{\delta}^{(0)}\right)^{2(\sigma-1)}}\right)^{2^{n+1}-2} \frac{1}{2} \mu_{7}^{(0)} \frac{\|U\|_{\bar{\rho}_{1}^{(0)}, \bar{\rho}_{2}^{(0)}}^{2}}{2^{4(\sigma-1) n}} \\
& =\left(\frac{\|U\|_{\bar{\rho}_{1}^{(0)}, \bar{\rho}_{2}^{(0)}}}{\bar{\Delta}}\right)^{2^{n+1}-2} \frac{1}{2} \mu_{7}^{(0)} \frac{\|U\|_{\bar{\rho}_{1}^{(0)}, \bar{\rho}_{2}^{(0)}}^{2}}{2^{4(\sigma-1) n}}
\end{aligned}
$$

with $\bar{\Delta}$ defined in (43). Thus, if we have that $\|U\|_{\bar{\rho}_{1}^{(0)}, \bar{\rho}_{2}^{(0)}} \leq \bar{\Delta}$, we can claim the wanted uniqueness.

\section{A.3 Proof of Theorem 3}

The next result to be considered is the proof of Theorem 3, that gives conditions to ensure convergence of the modified Newton method formulated in Section 4.

As we have mentioned before (see Section 2.3), despite the statement of Theorem 2 has been formulated for a "generic" set of $\omega$ and $V(q)$ (for which the conditions of the statement are fulfilled), in Theorem 3 we have adapted the application of the modified iterative method to the case in which we are interested, that is, the invariant curve with rotation number the golden mean of the standard map.

The proof of Theorem 3 will be done applying the modified Newton method to equation (85). This method has been constructed in Section 4 in terms of some variables called $\theta, u(\theta)$ and $\ell(\theta)$. Hence, in order to simplify the readability of the proof of Theorem 3 , as direct application of the formulation done in Section 4 , we will also use this notation during the proof, even though the statement has been done in terms of the variables $\tau, v(\tau)$, and $l(\tau)$. It is important to remark that now the variables $\theta$ and $\tau$ and the corresponding functions are not related by any change of variables, but they are just the same.

\section{A.3.1 Iterative lemma}

To prove Theorem 3 we follow the same basic scheme than in the proof of Theorem 2 . Then, first of all we will formulate an iterative lemma in order to control the bounds of the corrections of the functions $u^{(n)}(\theta), u^{\prime(n)}(\theta)$, and $w^{(n)}(\theta)$, of the constant $a^{(n)}$, as well the new errors $e^{(n+1)}(\theta), f^{(n+1)}(\theta)$, and after that, in Section A.3.2 we will prove the theorem.

Before to give the concrete statement of this (iterative) lemma, let us remark some technical details to be taken into account in the formulation of the result. Then, we resume the discussion that we have done in Section 5.2. We recall that we have to deal with several estimates on functions that become unbounded when we approach to the lower boundary of the corresponding strip of analyticity. To help in the control of the functions given rise to these estimates, we recall the notation introduced in Section 5.2. Then, given the domain $D=\mathcal{D}\left(\rho_{1}, \rho_{2}\right)$, and any $0<\delta \leq\left(\rho_{2}-\rho_{1}\right) / 2$, we put $\|\cdot\|_{D-\delta}=\|\cdot\|_{\rho_{1}+\delta, \rho_{2}-\delta}$. This notation will allow us to control the size of the functions close to the boundary of the domain (for small values of $\delta$ ), and also to control the size of its corresponding average (by choosing the biggest value for $\delta, \delta=\left(\rho_{2}-\rho_{1}\right) / 2$ ). We point that with this notation we are not able to distinguish the different behaviour of the function in the lower and upper boundary, and in fact, in our context everything can be more easily controlled in 
the upper boundary. However, we have preferred this approach instead of one carrying a separate control of both behaviours, as we only are interested to obtain careful estimates at the lower boundary. In the following lemma we summarize some properties related to this notation for the norm, that will be used in the sequel:

Lemma 6 Let $f(\theta)$ be an analytic function defined in $D=\mathcal{D}\left(\rho_{1}, \rho_{2}\right)$, for certain $-\infty \leq$ $\rho_{1}<\rho_{2} \leq+\infty$, and $2 \pi$-periodic in $\theta$. Then, for any $0<\delta_{0} \leq\left(\rho_{2}-\rho_{1}\right) / 2$, and for any $0 \leq \delta \leq\left(\rho_{2}-\rho_{1}-2 \delta_{0}\right) / 2$, we have:

- $\left\|f^{\prime}\right\|_{D-\delta-\delta_{0}} \leq \frac{\|f\|_{D-\delta / 2}}{\left(\delta / 2+\delta_{0}\right) \mathrm{e}}$

- If $\omega \in \mathbb{R}$ is a Diophantine number (4), and $\langle f\rangle=0$, then

$$
\left\|\mathcal{L}_{\omega}^{-1}(f)\right\|_{D-\delta_{0}-\delta} \leq \frac{1}{4 c_{\omega}}\left(\frac{\sigma-1}{\left(\delta / 2+\delta_{0}\right) \mathrm{e}}\right)^{\sigma-1}\|f\|_{D-\delta / 2} .
$$

Right after these remarks, we can formulate the result describing the effect of one step of the modified Newton method on a given approximation.

Lemma 7 We consider $\omega=2 \pi \gamma$, with $\gamma$ the golden mean, which is of Diophantine type (4), with $\sigma=2$ and for certain $c_{\gamma} \equiv c_{\omega}>0$. Let also be $V(q)$ a $2 \pi$-periodic entire function. Let $\ell^{(n)}(\theta)$ and $w^{(n)}(\theta)$ be analytic functions defined on $D^{(n)}=\mathcal{D}\left(\rho_{1}^{(n)}, \rho_{2}^{(n)}\right)$, for certain $-\infty<\rho_{1}^{(n)}<\rho_{2}^{(n)}<+\infty$, with $\left(\rho_{2}^{(n)}-\rho_{1}^{(n)}\right) / 2>1,2 \pi$-periodic in $\theta$ and with $\left\langle\ell^{(n)}\right\rangle=0$. We put $u^{(n)}(\theta)=\theta+\ell^{(n)}(\theta)$, and we assume that for certain $0<\delta^{(0)}<1$, and for any $0 \leq \delta \leq\left(\rho_{2}^{(n)}-\rho_{1}^{(n)}\right) / 2$, we have the following bounds:

$$
\begin{aligned}
&\left\|\ell^{(n)}\right\|_{D^{(n)}-\delta} \leq \mu_{1}^{(n)}, \quad\left\|u^{(n)}\right\|_{D^{(n)}-\delta} \leq \frac{\mu_{2}^{(n)}}{\delta+\delta^{(0)}}+1, \quad\left\|e^{(n)}\right\|_{D^{(n)}-\delta} \leq \mu_{3}^{(n)}, \\
&\left\|w^{(n)}\right\|_{D^{(n)}-\delta} \leq \mu_{4}^{(n)}\left[\frac{\log \left(\frac{1}{\delta+\delta^{(0)}}\right)}{\left(\delta+\delta^{(0)}\right)^{2}}+1\right], \quad 0<\mu_{5}^{(n)} \leq\left|a^{(n)}\right| \leq \mu_{6}^{(n)}, \\
&\left\|f^{(n)}\right\|_{D^{(n)}-\delta} \leq \mu_{9}^{(n)}, \quad\left\|V^{\prime \prime}\right\|_{D^{(n)}+\mu_{1}^{(0)}+1} \leq \mu_{7}^{(n)}, \quad\left\|V^{\prime \prime \prime}\right\|_{D^{(n)}+\mu_{1}^{(0)}+1} \leq \mu_{8}^{(n)},
\end{aligned}
$$

with $\mu_{j}^{(n)}<\mu_{j}^{(0)}+1$, for certain $\mu_{j}^{(0)}>0$, for $j=1,2,4$, and $\mu_{3}^{(n)} \leq \mu_{9}^{(n)}<1$, where $e^{(n)}(\theta)$ is defined in (54), $f^{(n)}(\theta)$ in (55) and $a^{(n)}$ is given in such a way that $\left\langle\operatorname{det}\left(P^{(n)}\right)\right\rangle=1$ in (57).

Then, there exists $c^{(n)} \geq 1, c^{(n)} \equiv c\left(\mu_{1}^{(0)}, \mu_{2}^{(0)}, \mu_{4}^{(0)}, 1 / \mu_{5}^{(n)}, \mu_{6}^{(n)}, \mu_{7}^{(n)}, \mu_{8}^{(n)}\right)$, that is an increasing function of all its arguments, such that given any $\delta^{(n)}$, with

$$
0<\delta^{(n)} \leq \min \left\{\delta^{(0)}, \frac{1}{5}\left(\frac{\rho_{2}^{(n)}-\rho_{1}^{(n)}}{2}-1\right)\right\},
$$

for which

$$
\begin{aligned}
c^{(n)} \frac{\mu_{3}^{(n)} \log \left(\frac{1}{\delta^{(0)}}\right)}{\left(\delta^{(0)}\right)^{2}\left(\delta^{(n)}\right)^{2}} & \leq \min \left\{1, \mu_{1}^{(0)}+1-\mu_{1}^{(n)}\right\}, \quad c^{(n)} \frac{\mu_{9}^{(n)}}{\left(\delta^{(n)}\right)^{2}} \leq \frac{\mu_{4}^{(0)}+1-\mu_{4}^{(n)}}{2}, \\
c^{(n)} \frac{\mu_{3}^{(n)} \log \left(\frac{1}{\delta^{(0)}}\right)}{\delta^{(0)}\left(\delta^{(n)}\right)^{3}} & \leq \min \left\{1, \mu_{2}^{(0)}+1-\mu_{2}^{(n)}\right\}, \quad c^{(n)} \mu_{9}^{(n)} \leq \frac{1}{2}, \\
c^{(n)} \frac{\mu_{3}^{(n)}\left(\log \left(\frac{1}{\delta^{(0)}}\right)\right)^{2}}{\left(\delta^{(0)}\right)^{3}\left(\delta^{(n)}\right)^{5}} & \leq \frac{\mu_{4}^{(0)}+1-\mu_{4}^{(n)}}{2},
\end{aligned}
$$


then we can apply a step of the modified Newton method to compute a new iterate given by $u^{(n+1)}(\theta)=\theta+\ell^{(n+1)}(\theta)$, with $\left\langle\ell^{(n+1)}\right\rangle=0, w^{(n+1)}(\theta)$ and $a^{(n+1)}$, with the estimates

$$
\begin{aligned}
\left\|\ell^{(n+1)}(\theta)-\ell^{(n)}(\theta)\right\|_{D^{(n+1)}-\delta} & \leq c^{(n)} \frac{\mu_{3}^{(n)} \log \left(\frac{1}{\delta^{(0)}}\right)}{\left(\delta^{(0)}\right)^{2}\left(\delta^{(n)}\right)^{2}} \\
\left\|\ell^{(n+1)}(\theta)-\ell^{\prime(n)}(\theta)\right\|_{D^{(n+1)}-\delta} & \leq c^{(n)} \frac{\mu_{3}^{(n)} \log \left(\frac{1}{\delta^{(0)}}\right)}{\delta^{(0)}\left(\delta^{(n)}\right)^{3}} \frac{1}{\delta+\delta^{(0)}}, \\
\left\|w^{(n+1)}(\theta)-w^{(n)}(\theta)\right\|_{D^{(n+1)}-\delta} & \leq c^{(n)}\left[\frac{\mu_{9}^{(n)}}{\left(\delta^{(n)}\right)^{2}}+\frac{\mu_{3}^{(n)}\left(\log \left(\frac{1}{\delta^{(0)}}\right)\right)^{2}}{\left(\delta^{(0)}\right)^{3}\left(\delta^{(n)}\right)^{5}}\right]\left(\frac{\log \left(\frac{1}{\delta+\delta^{(0)}}\right)}{\left(\delta+\delta^{(0)}\right)^{2}}+1\right), \\
\left|a^{(n+1)}-a^{(n)}\right| & \leq c^{(n)} \mu_{9}^{(n)},
\end{aligned}
$$

that hold for any $0 \leq \delta \leq\left(\rho_{2}^{(n+1)}-\rho_{1}^{(n+1)}\right) / 2$, where $\rho_{2}^{(n+1)}=\rho_{2}^{(n)}-5 \delta^{(n)}, \rho_{1}^{(n+1)}=$ $\rho_{1}^{(n)}+5 \delta^{(n)}$ and $D^{(n+1)}=\mathcal{D}\left(\rho_{1}^{(n+1)}, \rho_{2}^{(n+1)}\right)$. Moreover, if we replace $u^{(n)}(\theta), w^{(n)}(\theta)$ and $a^{(n)}$ by the corrected expressions $a^{(n+1)}, u^{(n+1)}(\theta)$ and $w^{(n+1)}(\theta)$, we have that $\left\langle\operatorname{det}\left(P^{(n+1)}\right)\right\rangle=1$ in (56), and the following bounds for the new errors $e^{(n+1)}(\theta)$ and $f^{(n+1)}(\theta)$ (see (54) and (55)):

$$
\begin{aligned}
& \left\|e^{(n+1)}\right\|_{D^{(n+1)}-\delta} \leq c^{(n)} \frac{\mu_{3}^{(n)}}{\delta^{(0)} \delta^{(n)}}\left[\frac{\mu_{3}^{(n)}\left(\log \left(\frac{1}{\delta^{(0)}}\right)\right)^{2}}{\left(\delta^{(0)}\right)^{3}\left(\delta^{(n)}\right)^{3}}+\mu_{9}^{(n)}\right] \\
& \left\|f^{(n+1)}\right\|_{D^{(n+1)}-\delta} \leq c^{(n)} \frac{1}{\delta^{(0)} \delta^{(n)}}\left[\frac{\mu_{3}^{(n)}\left(\log \left(\frac{1}{\delta^{(0)}}\right)\right)^{2}}{\left(\delta^{(0)}\right)^{3}\left(\delta^{(n)}\right)^{3}}+\mu_{9}^{(n)}\right]^{2} .
\end{aligned}
$$

Proof: To prove this result we will do an analogous simplification on the notation than in the proof of Lemma 5 , that is, to skip the super-script $(n)$ of the n-iteration and to use the super-script (1) to label the $(n+1)$ iteration. However, in the present context we will let the super-script $(n)$ for the quantity $\delta^{(n)}$ controlling the reduction of the domain at the $n$-step, in order to distinguish it from $\delta$, that is used to display the bounds as function to the distance to the boundary. Moreover, the role played by the constant $c$ of the statement is the same than in the iterative Lemma 5. More concretely, this constant will be re-defined in a recurrent way (but keeping always the same name) to achieve the different bounds appearing in the proof and that are function of the arguments in which $c$ depends. Then, the value for $c$ in the statement is the one that follows from the last bound.

It is important to note that, in the bounds that will appear during the proof, we will keep only the dominant terms with respect to $\delta$, even for small and for big values of $\delta$.

First of all, we solve (63) and we compute $\xi(\theta)$ from (62). Then, we have

$$
\begin{aligned}
\left\|u^{\prime}(\theta) e(\theta)\right\|_{D-\delta} & \leq c \mu_{3}\left(\frac{1}{\delta^{(0)}+\delta}+1\right) \\
\|w(\theta) e(\theta)\|_{D-\delta} & \leq c \mu_{3}\left(\frac{\log \left(\frac{1}{\delta+\delta^{(0)}}\right)}{\left(\delta+\delta^{(0)}\right)^{2}}+1\right) \\
|\langle w e\rangle| & \leq c \mu_{3} . \\
\left\|\widetilde{\eta}_{2}(\theta)\right\|_{D-\delta^{(n)}-\delta} & \leq \frac{c \mu_{3}}{\delta^{(n)}+\delta / 2}\left(\frac{1}{\delta^{(0)}+\delta / 2}+1\right),
\end{aligned}
$$




$$
\begin{aligned}
\left|\left\langle\eta_{2}\right\rangle\right| & \leq c \mu_{3} \\
\left\|\eta_{2}(\theta)\right\|_{D-\delta^{(n)}-\delta} & \leq c \mu_{3}\left(\frac{1}{\left(\delta^{(0)}+\delta / 2\right)\left(\delta^{(n)}+\delta / 2\right)}+1\right) \\
\left\|\widetilde{\eta}_{1}(\theta)\right\|_{D-2 \delta^{(n)}-\delta} & \leq \frac{c \mu_{3}}{\delta^{(n)}+\delta / 2}\left(\frac{\log \left(\frac{1}{\delta^{(0)}+\delta / 2}\right)}{\left(\delta^{(0)}+\delta / 4\right)\left(\delta^{(n)}+\delta / 4\right)}+\frac{1}{\delta^{(n)}+\delta / 4}\right) \\
\left|\left\langle u^{\prime} \tilde{\eta}_{1}\right\rangle\right| & \leq c \mu_{3} \\
\left|\left\langle w \eta_{2}\right\rangle\right| & \leq c \mu_{3} \\
\left|\left\langle\eta_{1}\right\rangle\right| & \leq c \mu_{3} \\
\left\|\eta_{1}(\theta)\right\|_{D-2 \delta^{(n)}-\delta} & \leq c \mu_{3}\left(\frac{\log \left(\frac{1}{\delta^{(0)}+\delta / 2}\right)}{\left(\delta^{(0)}+\delta / 4\right)\left(\delta^{(n)}+\delta / 4\right)^{2}}+1\right) \\
\|\xi(\theta)\|_{D-2 \delta^{(n)}-\delta} & \leq c \mu_{3}\left(\frac{\log \left(\frac{1}{\delta^{(0)}+\delta / 2}\right)}{\left(\delta^{(0)}+\delta / 4\right)^{2}\left(\delta^{(n)}+\delta / 4\right)^{2}}+1\right) \\
\left\|\xi^{\prime}(\theta)\right\|_{D-3 \delta^{(n)}-\delta} & \leq \frac{c \mu_{3}}{\delta / 2+\delta^{(n)}}\left(\frac{\log \left(\frac{1}{\delta^{(0)}+\delta / 4}\right)}{\left(\delta^{(0)}+\delta / 8\right)^{2}\left(\delta^{(n)}+\delta / 8\right)^{2}}+1\right)
\end{aligned}
$$

Thus, these bounds imply the new bounds for $\left\|\ell^{(1)}(\theta)-\ell(\theta)\right\|$ and $\left\|\ell^{\prime(1)}(\theta)-\ell^{\prime}(\theta)\right\|$ given in (107). To bound $e^{(1)}(\theta)$, we go back to the decomposition

$$
e^{(1)}(\theta)=e_{1}^{(1)}(\theta)+e_{2}^{(1)}(\theta)
$$

that we have done in (33), but now we have to use (34) for $e_{2}^{(1)}(\theta)$ and (79) for $e_{1}^{(1)}(\theta)$. The estimate for $e_{1}^{(1)}(\theta)$ follows directly. For $e_{2}^{(1)}(\theta)$, we remark that from the hypotheses (106) on the statement, we have $\left\|\ell^{(1)}(\theta)\right\|_{D-2 \delta^{(n)}} \leq \mu_{1}^{(0)}+1$. Then, we can use the bound for the remainder of the Taylor formula up to order 1 given in Lemma 4 in the domain $\mathcal{D}\left(\rho_{1}-\mu_{1}^{(0)}-1, \rho_{2}+\mu_{1}^{(0)}+1\right)$, obtaining

$$
\left\|e_{1}^{(1)}(\theta)\right\|_{D-2 \delta^{(n)}-\delta} \leq c \mu_{3}\left(\frac{\mu_{3} \log \left(\frac{1}{\delta^{(0)}+\delta / 2}\right)}{\left(\delta^{(0)}+\delta / 4\right)\left(\delta^{(n)}+\delta / 4\right)^{3}}+\frac{\mu_{9}}{\left(\delta^{(0)}+\delta / 2\right)\left(\delta^{(n)}+\delta / 2\right)}+\mu_{9}\right),
$$

and

$$
\left\|e_{2}^{(1)}(\theta)\right\|_{D-2 \delta^{(n)}-\delta} \leq c \mu_{3}^{2}\left(\frac{\left(\log \left(\frac{1}{\delta^{(0)}+\delta / 2}\right)\right)^{2}}{\left(\delta^{(0)}+\delta / 4\right)^{4}\left(\delta^{(n)}+\delta / 4\right)^{4}}+1\right) .
$$

Then, we obtain

$$
\left\|e^{(1)}(\theta)\right\|_{D-2 \delta^{(n)}-\delta} \leq c \mu_{3}\left(\frac{\mu_{3}\left(\log \left(\frac{1}{\delta^{(0)}+\delta / 2}\right)\right)^{2}}{\left(\delta^{(0)}+\delta / 4\right)^{4}\left(\delta^{(n)}+\delta / 4\right)^{4}}+\frac{\mu_{9}}{\left(\delta^{(0)}+\delta / 2\right)\left(\delta^{(n)}+\delta / 2\right)}+\mu_{9}\right)
$$

and from here, we derive bound (108).

At this point, and in order to bound the new iterate $w^{(n+1)}(\theta)$ and its associated error $f^{(n+1)}(\theta)$, we need some intermediate bounds. From (67), and using the "mean value 
theorem" given in Lemma 4, we obtain

$$
\|g(\theta)\|_{D-3 \delta^{(n)}-\delta} \leq c\left(\frac{\mu_{3}\left(\log \left(\frac{1}{\delta^{(0)}+\delta / 4}\right)\right)^{2}}{\left(\delta^{(0)}+\delta / 8\right)^{3}\left(\delta^{(n)}+\delta / 8\right)^{3}}+\mu_{9}\right) .
$$

Going back to the system of equations (73), that define $\alpha(\theta)$ and $\beta(\theta)$, we have

$$
\left\|e^{\prime(1)}(\theta) w(\theta)-u^{\prime(1)}(\theta) g(\theta)\right\|_{D-3 \delta^{(n)}-\delta} \leq c\left(\frac{\mu_{3}\left(\log \left(\frac{1}{\delta^{(0)}+\delta / 2}\right)\right)^{2}}{\left(\delta^{(0)}+\delta / 8\right)^{4}\left(\delta^{(n)}+\delta / 8\right)^{3}}+\frac{\mu_{9}}{\delta^{(0)}+\delta}+\mu_{9}\right),
$$

where we have used that the hypotheses (106) on the statement guarantee that we have for $u^{\prime(1)}(\theta)$ the same estimate than for $u^{\prime}(\theta)$, just replacing $\mu_{2}$ by $\mu_{2}^{(0)}+1$ in the domain $D-2 \delta^{(n)}-\delta$, and also that the contribution due to the "quadratic terms" $e^{(1)}(\theta) w(\theta)$ can be controlled by the one due to $u^{\prime(1)}(\theta) g(\theta)$. Moreover, we also have

$$
\begin{aligned}
\|w(\theta) g(\theta)\|_{D-3 \delta^{(n)}-\delta} & \leq c\left(\frac{\mu_{3}\left(\log \left(\frac{1}{\delta^{(0)}+\delta / 4}\right)\right)^{3}}{\left(\delta^{(0)}+\delta / 8\right)^{5}\left(\delta^{(n)}+\delta / 8\right)^{3}}+\frac{\mu_{9} \log \left(\frac{1}{\delta^{(0)}+\delta}\right)}{\left(\delta^{(0)}+\delta\right)^{2}}+\mu_{9}\right) \\
|\langle w g\rangle| & \leq c \mu_{9}
\end{aligned}
$$

and so,

$$
\begin{aligned}
&\|\tilde{\beta}(\theta)\|_{D-4 \delta^{(n)}-\delta} \leq \frac{c}{\delta^{(n)}+\delta / 2}\left(\frac{\mu_{3}\left(\log \left(\frac{1}{\delta^{(0)}+\delta / 4}\right)\right)^{2}}{\left(\delta^{(0)}+\delta / 16\right)^{4}\left(\delta^{(n)}+\delta / 16\right)^{3}}+\frac{\mu_{9}}{\delta^{(0)}+\delta / 2}+\mu_{9}\right) \\
&|\langle\beta\rangle| \leq c \mu_{9} \\
&\|\beta(\theta)\|_{D-4 \delta^{(n)}-\delta} \leq c\left(\frac{\mu_{3}\left(\log \left(\frac{1}{\delta^{(0)}+\delta / 4}\right)\right)^{2}}{\left(\delta^{(0)}+\delta / 16\right)^{4}\left(\delta^{(n)}+\delta / 16\right)^{4}}+\frac{\mu_{9}}{\left(\delta^{(0)}+\delta / 2\right)\left(\delta^{(n)}+\delta / 2\right)}+\mu_{9}\right), \\
&\|\alpha(\theta)\|_{D-5 \delta^{(n)}-\delta \leq} \leq \frac{c}{\delta^{(n)}+\delta / 2}\left(\frac{\mu_{3}\left(\log \left(\frac{1}{\delta^{(0)}+\delta / 8}\right)\right)^{3}}{\left(\delta^{(0)}+\delta / 32\right)^{4}\left(\delta^{(n)}+\delta / 32\right)^{4}}\right. \\
&\left.+\frac{\mu_{9} \log \left(\frac{1}{\delta^{(0)}+\delta / 2}\right)}{\left(\delta^{(0)}+\delta / 4\right)\left(\delta^{(n)}+\delta / 4\right)}+\mu_{9}\right) .
\end{aligned}
$$

Then, we have for $\widehat{\chi}(\theta)$ (see $(69))$ :

$$
\|\widehat{\chi}(\theta)\|_{D-5 \delta^{(n)}-\delta} \leq c\left(\frac{\mu_{3}\left(\log \left(\frac{1}{\delta^{(0)}+\delta / 8}\right)\right)^{3}}{\left(\delta^{(0)}+\delta / 32\right)^{5}\left(\delta^{(n)}+\delta / 32\right)^{5}}+\frac{\mu_{9} \log \left(\frac{1}{\delta^{(0)}+\delta / 2}\right)}{\left(\delta^{(0)}+\delta / 4\right)^{2}\left(\delta^{(n)}+\delta / 4\right)^{2}}+\mu_{9}\right) .
$$

We point that all these bounds are valid for any value of $\delta$, not necessarily small, with the only restriction that $0 \leq \delta \leq\left(\rho_{2}-\rho_{1}-10 \delta^{(n)}\right) / 2$.

In order to bound the new iterate $w^{(1)}(\theta)$, we need to control the average of the determinant of the intermediate Floquet matrix $\widehat{P}(\theta)$ defined in (75). We can do this if 
we put the maximum value allowed for $\delta$ in the bounds of $\xi(\theta)$ and $\widehat{\chi}(\theta)$. Then, using condition (105) for $\delta^{(n)}$, we obtain that $\Delta \hat{d}(\theta)$ (defined in (76)) verifies:

$$
|\langle\Delta \widehat{d}\rangle| \leq c \mu_{9}
$$

that is bounded by $1 / 2$ by the hypotheses $(106)$ of the statement. So, for $w^{(1)}(\theta)$, we have:

$$
\begin{aligned}
& \left\|w^{(1)}(\theta)-w(\theta)\right\|_{D-5 \delta^{(n)}-\delta}=\left\|\frac{\hat{\chi}(\theta)-\langle\Delta \widehat{d}\rangle w(\theta)}{1+\langle\Delta \widehat{d}\rangle}\right\|_{D-5 \delta^{(n)}-\delta} \\
& \leq c\left(\frac{\mu_{3}\left(\log \left(\frac{1}{\delta^{(0)}+\delta / 8}\right)\right)^{3}}{\left(\delta^{(0)}+\delta / 32\right)^{5}\left(\delta^{(n)}+\delta / 32\right)^{5}}+\frac{\mu_{9} \log \left(\frac{1}{\delta^{(0)}+\delta / 2}\right)}{\left(\delta^{(0)}+\delta / 4\right)^{2}\left(\delta^{(n)}+\delta / 4\right)^{2}}+\mu_{9}\right) .
\end{aligned}
$$

From here, we deduce the bound given in (107) for the new function $w^{(1)}(\theta)$, and then, conditions (106) on the statement guarantee that $\left\|w^{(1)}(\theta)\right\|_{D-5 \delta^{(n)}-\delta}$ verifies the same estimate than $\|w(\theta)\|_{D-\delta}$ in (104), just replacing $\mu_{4}$ by $\mu_{4}^{(0)}+1$. Moreover, equation (77) gives that

$$
\left|a^{(1)}-a\right|=\frac{|\langle\Delta \widehat{d}\rangle||a|}{|1+\langle\Delta \widehat{d}\rangle|} \leq c \mu_{9}
$$

Now, it only remains to bound the error of $w^{(1)}(\theta)$ in (55) that is given by formulae (78) and (84). To do that, we also need several intermediate bounds. First of all, from (59), we have

$$
\|d(\theta)\|_{D-2 \delta^{(n)}-\delta} \leq \frac{c}{\delta^{(n)}+\delta / 2}\left(\frac{\mu_{3} \log \left(\frac{1}{\delta^{(0)}+\delta / 2}\right)}{\left(\delta^{(0)}+\delta / 2\right)^{2}\left(\delta^{(n)}+\delta / 4\right)}+\frac{\mu_{9}}{\delta^{(0)}+\delta / 2}+\mu_{9}\right) .
$$

We recall that we have written $1-\operatorname{det}(\bar{P}(\theta))=\Delta \bar{d}(\theta)-d(\theta)$, with $\bar{P}(\theta)$ introduced in (64) and $\Delta \bar{d}(\theta)$ defined in (83). For $\Delta \bar{d}(\theta)$ we have

$$
\|\Delta \bar{d}(\theta)\|_{D-3 \delta^{(n)}-\delta} \leq \frac{c \mu_{3}}{\delta^{(n)}+\delta / 2}\left(\frac{\left(\log \left(\frac{1}{\delta^{(0)}+\delta / 4}\right)\right)^{2}}{\left(\delta^{(0)}+\delta / 8\right)^{4}\left(\delta^{(n)}+\delta / 8\right)^{2}}+1\right) .
$$

Thus, we obtain

$$
\|1-\operatorname{det} \bar{P}(\theta)\|_{D-3 \delta^{(n)}-\delta} \leq \frac{c}{\delta^{(n)}+\delta / 2}\left(\frac{\mu_{3}\left(\log \left(\frac{1}{\delta^{(0)}+\delta / 4}\right)\right)^{2}}{\left(\delta^{(0)}+\delta / 8\right)^{4}\left(\delta^{(n)}+\delta / 8\right)^{2}}+\frac{\mu_{9}}{\delta^{(0)}+\delta / 2}+\mu_{9}\right) .
$$

Using this bound on (82), we have

$$
\begin{aligned}
\left\|\hat{e}_{2}(\theta)\right\|_{D-5 \delta^{(n)}-\delta} \leq & c\left(\frac{\mu_{3}^{2}\left(\log \left(\frac{1}{\delta^{(0)}+\delta / 4}\right)\right)^{4}}{\left(\delta^{(0)}+\delta / 16\right)^{7}\left(\delta^{(n)}+\delta / 16\right)^{7}}+\frac{\mu_{3} \mu_{9}\left(\log \left(\frac{1}{\delta^{(0)}+\delta / 4}\right)\right)^{2}}{\left(\delta^{(0)}+\delta / 16\right)^{4}\left(\delta^{(n)}+\delta / 16\right)^{4}}\right. \\
& \left.+\frac{\mu_{9}^{2}}{\left(\delta^{(0)}+\delta / 2\right)\left(\delta^{(n)}+\delta / 2\right)}+\mu_{9}^{2}\right) .
\end{aligned}
$$


Here, the hypotheses (106) allows to control the terms $e^{\prime(1)}(\theta) \alpha(\theta)$ and $e^{\prime(1)}(\theta) \beta(\theta)$ ), which have "cubic size" in $\mu_{3}$ and $\mu_{9}$, by means of the size of the other ones. On the other hand, formula (81) gives

$$
\begin{aligned}
\left\|\widehat{e}_{1}(\theta)\right\|_{D-3 \delta^{(n)}-\delta} \leq & c\left(\frac{\mu_{3}^{2}\left(\log \left(\frac{1}{\delta^{(0)}+\delta / 4}\right)\right)^{4}}{\left(\delta^{(0)}+\delta / 8\right)^{8}\left(\delta^{(n)}+\delta / 8\right)^{5}}+\frac{\mu_{3} \mu_{9}\left(\log \left(\frac{1}{\delta^{(0)}+\delta}\right)\right)^{2}}{\left(\delta^{(0)}+\delta / 4\right)^{5}\left(\delta^{(n)}+\delta / 4\right)^{2}}\right. \\
& \left.+\frac{\mu_{3} \mu_{9}}{\delta^{(n)}+\delta / 2}\right) .
\end{aligned}
$$

Then, by (84)

$$
\begin{aligned}
\|\widehat{f}(\theta)\|_{D-5 \delta^{(n)}-\delta} \leq & c\left(\frac{\mu_{3}^{2}\left(\log \left(\frac{1}{\delta^{(0)}+\delta / 4}\right)\right)^{4}}{\left(\delta^{(0)}+\delta / 16\right)^{7}\left(\delta^{(n)}+\delta / 16\right)^{7}}+\frac{\mu_{3} \mu_{9}\left(\log \left(\frac{1}{\delta^{(0)}+\delta / 4}\right)\right)^{2}}{\left(\delta^{(0)}+\delta / 16\right)^{4}\left(\delta^{(n)}+\delta / 16\right)^{4}}\right. \\
& \left.+\frac{\mu_{9}^{2}}{\left(\delta^{(0)}+\delta / 2\right)\left(\delta^{(n)}+\delta / 2\right)}+\mu_{9}^{2}\right) .
\end{aligned}
$$

Now, from the definition of $f^{(1)}(\theta)$ in $(78)$, and using again that $|\langle\Delta \widehat{d}\rangle| \leq 1 / 2$, the bound (108) claimed in the statement for $f^{(1)}(\theta)$ follows.

\section{A.3.2 Proof of the convergence}

In this section we are going to prove Theorem 3, showing the convergence of the sequences $u^{(n)}(\theta), w^{(n)}(\theta)$ and $a^{(n)}$ constructed in Section 4. We will proof this convergence by applying iteratively Lemma 7 , taking $\delta^{(n)}=\delta^{(0)} / 2^{n}$, and showing that $u^{(n)}(\theta), w^{(n)}(\theta)$ and $a^{(n)}$ are small perturbations of $u^{(0)}(\theta), w^{(0)}(\theta)$ and $a^{(0)}$.

Now, we proceed by induction. We assume done $n$ steps of the iterative method, and that for $u^{(n)}(\theta), w^{(n)}(\theta)$ and $a^{(n)}$ the constants $\mu_{j}^{(n)}, j=1, \cdots, 9$, defined in (104) verify $\mu_{j}^{(n)}<\mu_{j}^{(0)}+1$, for $j=1,2,4,1 / \mu_{5}^{(n)} \leq 2 / \mu_{5}^{(0)}, \mu_{6}^{(n)} \leq 2 \mu_{6}^{(0)}$ and $\mu_{3}^{(n)} \leq \mu_{9}^{(n)}<1$. We note that conditions (93) and (95) assure the last inequality when $n=0$. On the other hand, it is clear that $\mu_{j}^{(n)} \leq \mu_{j}^{(0)}$, for $j=7,8$. We also assume that

$$
\begin{aligned}
c \frac{\mu_{3}^{(n)} \log \left(\frac{1}{\delta^{(0)}}\right)}{\left(\delta^{(0)}\right)^{2}\left(\delta^{(n)}\right)^{2}} & <\min \left\{1, \mu_{1}^{(0)}+1-\mu_{1}^{(n)}\right\} \\
c \frac{\mu_{9}^{(n)}}{\left(\delta^{(n)}\right)^{2}} & <\frac{\mu_{4}^{(0)}+1-\mu_{4}^{(n)}}{2} \\
c \frac{\mu_{3}^{(n)} \log \left(\frac{1}{\delta^{(0)}}\right)}{\delta^{(0)}\left(\delta^{(n)}\right)^{3}} & <\min \left\{1, \mu_{2}^{(0)}+1-\mu_{2}^{(n)}\right\} \\
c \frac{\mu_{3}^{(n)}\left(\log \left(\frac{1}{\delta^{(0)}}\right)\right)^{2}}{\left(\delta^{(0)}\right)^{3}\left(\delta^{(n)}\right)^{5}} & <\frac{\mu_{4}^{(0)}+1-\mu_{4}^{(n)}}{2}, \\
c \mu_{9}^{(n)} & <\min \left\{\frac{1}{2}, \mu_{5}^{(n)}-\frac{\mu_{5}^{(0)}}{2}, 2 \mu_{6}^{(0)}-\mu_{6}^{(n)}\right\},
\end{aligned}
$$

where $c$ is the function provided by Lemma 7 evaluated in

$$
c \equiv c\left(\mu_{1}^{(0)}, \mu_{2}^{(0)}, \mu_{4}^{(0)}, 2 / \mu_{5}^{(0)}, 2 \mu_{6}^{(0)}, \mu_{7}^{(0)}, \mu_{8}^{(0)}\right) .
$$


So, from the inductive hypotheses we have that $c^{(j)} \leq c$ for any $j \leq n$. Moreover, from the recursive definition of $\rho_{1}^{(n)}$ and $\rho_{2}^{(n)}$ (see Lemma 7), and using hypothesis (94) on the statement, we also have that

$$
\frac{\rho_{2}^{(n)}-\rho_{1}^{(n)}}{2}>\frac{\rho_{2}^{(0)}-\rho_{1}^{(0)}-20 \delta^{(0)}}{2} \geq 1
$$

for any $n \geq 0$. Then, conditions (109) imply that (106) is fulfilled for the present $n$ and Lemma 7 can be applied, giving:

$$
\begin{aligned}
& \mu_{1}^{(n+1)}=\mu_{1}^{(n)}+c \frac{\mu_{3}^{(n)} \log \left(\frac{1}{\delta^{(0)}}\right)}{\left(\delta^{(0)}\right)^{2}\left(\delta^{(n)}\right)^{2}} \\
& \mu_{2}^{(n+1)}=\mu_{2}^{(n)}+c \frac{\mu_{3}^{(n)} \log \left(\frac{1}{\delta^{(0)}}\right)}{\delta^{(0)}\left(\delta^{(n)}\right)^{3}} \\
& \mu_{3}^{(n+1)}=c \frac{\mu_{3}^{(n)}}{\delta^{(0)} \delta^{(n)}}\left(\frac{\mu_{3}^{(n)}\left(\log \left(\frac{1}{\delta^{(0)}}\right)\right)^{2}}{\left(\delta^{(0)}\right)^{3}\left(\delta^{(n)}\right)^{3}}+\mu_{9}^{(n)}\right), \\
& \mu_{4}^{(n+1)}=\mu_{4}^{(n)}+c\left(\frac{\mu_{9}^{(n)}}{\left(\delta^{(n)}\right)^{2}}+\frac{\mu_{3}^{(n)}\left(\log \left(\frac{1}{\delta^{(0)}}\right)\right)^{2}}{\left(\delta^{(0)}\right)^{3}\left(\delta^{(n)}\right)^{5}}\right), \\
& \mu_{5}^{(n+1)}=\mu_{5}^{(n)}-c \mu_{9}^{(n)}, \\
& \mu_{6}^{(n+1)}=\mu_{6}^{(n)}+c \mu_{9}^{(n)}, \\
& \mu_{9}^{(n+1)}=c \frac{\mu_{3}^{(n)}}{\delta^{(0)} \delta^{(n)}}\left(\frac{\mu_{3}^{(n)}\left(\log \left(\frac{1}{\delta^{(0)}}\right)\right)^{2}}{\left(\delta^{(0)}\right)^{3}\left(\delta^{(n)}\right)^{3}}+\mu_{9}^{(n)}\right)^{2} .
\end{aligned}
$$

Moreover, conditions (109) give that $\mu_{j}^{(n+1)}<\mu_{j}^{(0)}+1$, for $j=1,2,4, \mu_{5}^{(n+1)}>\mu_{5}^{(0)} / 2$ and $\mu_{6}^{(n+1)}<2 \mu_{6}^{(0)}$.

As we want to prove convergence, the first thing we have to do is to study the behaviour of the errors, that in this modified method come from two different sources, given by the constants $\mu_{3}^{(n)}$ and $\mu_{9}^{(n)}$. In order to control both simultaneously, let us define a new error constant

$$
\mu_{10}^{(n)}=\frac{\mu_{3}^{(n)}\left(\log \left(\frac{1}{\delta^{(0)}}\right)\right)^{2}}{\left(\delta^{(0)} \delta^{(n)}\right)^{3}}+\mu_{9}^{(n)},
$$

which, from (110), verifies

$$
\mu_{10}^{(n+1)} \leq \frac{2 c}{\delta^{(0)} \delta^{(n)}}\left(\mu_{10}^{(n)}\right)^{2}
$$

So, we can control $\mu_{10}^{(j)}$, for any $j \leq n$, having

$$
\mu_{10}^{(j+1)} \leq \frac{c 2^{j+1}}{\left(\delta^{(0)}\right)^{2}}\left(\mu_{10}^{(j)}\right)^{2}
$$

Using the expressions for $\mu_{1}^{(0)}$ and $\mu_{2}^{(0)}$ given in (93), we can take

$$
\mu_{10}^{(0)}=\frac{3 \mu_{3}^{(0)}\left(\log \left(\frac{1}{\delta^{(0)}}\right)\right)^{2}}{\left(\delta^{(0)}\right)^{6}},
$$


and then, from the definition of $\Delta$ on the statement, we have

$$
\mu_{10}^{(j)} \leq \frac{\mu_{10}^{(0)}}{2^{j}}\left(\frac{4 c \mu_{10}^{(0)}}{\left(\delta^{(0)}\right)^{2}}\right)^{2^{j}-1}=\frac{3 \mu_{3}^{(0)}\left(\log \left(\frac{1}{\delta^{(0)}}\right)\right)^{2}}{2^{j}\left(\delta^{(0)}\right)^{6}} \Delta^{2^{j}-1}
$$

Then, as $\Delta \leq 1$ by hypotheses (95), we can assure that the bound of $\mu_{10}^{(j)}$ is strictly decreasing to zero with $j$. Moreover, the same happens for the bounds of the errors $\mu_{3}^{(j)}$ and $\mu_{9}^{(j)}$, as we have for $\mu_{9}^{(j)}$ the same bound than for $\mu_{10}^{(j)}$, and

$$
\mu_{3}^{(j)} \leq \frac{3 \mu_{3}^{(0)}}{2^{4 j}} \Delta^{2^{j}-1}
$$

This implies that we can keep iteratively the condition $\mu_{3}^{(n)} \leq \mu_{9}^{(n)} \leq 1$. Now, we only have to use the induction to control the remaining constants $\mu_{j}^{(n+1)}$. For instance, for $\mu_{1}^{(n+1)}$ we have that

$$
\begin{aligned}
\mu_{1}^{(n+1)} & \leq \mu_{1}^{(0)}+\sum_{j=0}^{n} c \frac{\mu_{3}^{(j)} \log \left(\frac{1}{\delta^{(0)}}\right)}{\left(\delta^{(0)}\right)^{2}\left(\delta^{(j)}\right)^{2}} \leq \mu_{1}^{(0)}+c \frac{3 \mu_{3}^{(0)} \log \left(\frac{1}{\delta^{(0)}}\right)}{\left(\delta^{(0)}\right)^{4}} \sum_{j=0}^{n} \frac{\Delta^{2^{j}-1}}{2^{2 j}} \\
& <\mu_{1}^{(0)}+6 c \frac{\mu_{3}^{(0)} \log \left(\frac{1}{\delta^{(0)}}\right)}{\left(\delta^{(0)}\right)^{4}}
\end{aligned}
$$

where we have bounded the sum by a geometric one of ratio $\Delta / 4$, using that $\Delta / 4 \leq 1 / 2$ by the hypotheses (95). Repeating the same process for the other constants, we also obtain that

$$
\begin{aligned}
& \mu_{2}^{(n+1)}<\mu_{2}^{(0)}+6 c \frac{\mu_{3}^{(0)} \log \left(\frac{1}{\delta^{(0)}}\right)}{\left(\delta^{(0)}\right)^{4}}, \quad \mu_{4}^{(n+1)}<\mu_{4}^{(0)}+6 c \frac{\mu_{3}^{(0)}\left(\log \left(\frac{1}{\delta^{(0)}}\right)\right)^{2}}{\left(\delta^{(0)}\right)^{8}} \\
& \mu_{5}^{(n+1)}>\mu_{5}^{(0)}-6 c \frac{\mu_{3}^{(0)}\left(\log \left(\frac{1}{\delta^{(0)}}\right)\right)^{2}}{\left(\delta^{(0)}\right)^{6}}, \quad \mu_{6}^{(n+1)}>\mu_{6}^{(0)}+6 c \frac{\mu_{3}^{(0)}\left(\log \left(\frac{1}{\delta^{(0)}}\right)\right)^{2}}{\left(\delta^{(0)}\right)^{6}}
\end{aligned}
$$

where we have compared the corresponding sums by geometric ones of ratios $\Delta / 2,2 \Delta$, $\Delta / 2$ and $\Delta / 2$, respectively. In any of these cases, from (95), the ratio is not bigger than $1 / 2$. Now, also using (95), we can assure that conditions (109) are verified iteratively, and hence, we can take $n \rightarrow+\infty$. So, the convergence follows immediately, and we also obtain the bounds for the limit claimed in the statement.

\section{A.4 Some sharp estimates on the small divisors for the golden mean}

The only thing that remains to end the paper is to prove the estimates on the small divisors associated to the golden mean, that have been used to prove Lemma 2 . The concrete result that we need is stated in Lemma 8. Let us point that this estimate can be deduced from a more general result stated in [13]. Nevertheless, in [13] this result is used without giving the proof, that seems to be not available anywhere. So, for this reason, and in order to make a self-contained paper, we have decided to include the proof of this result, but only in the concrete case of the golden mean. 
Lemma 8 We consider $\gamma=(\sqrt{5}-1) / 2$ the golden mean. Then, there exists a constant $K>0$, such that for any $M \in \mathbb{N}$ we have

$$
\sum_{m=1}^{M} \max _{n \in \mathbb{Z}}\left\{|m \gamma-n|^{-1}\right\} \leq K M \log (M+1) .
$$

The proof of this result follows as consequence of the following auxiliary results, which are devoted to study some basic properties of the Fibonacci numbers:

$$
F_{0}=1, \quad F_{1}=1, \quad F_{k+1}=F_{k}+F_{k-1}, k \geq 1
$$

or in an explicit way:

$$
F_{k}=A\left(\frac{1}{\gamma}\right)^{k}+B(-\gamma)^{k}, \quad A=\frac{1}{1+\gamma^{2}}, \quad B=\frac{\gamma^{2}}{1+\gamma^{2}}
$$

Lemma 9 For any $m \in \mathbb{N}, m$ can be decomposed as sum of non-consecutive Fibonacci numbers. That is,

$$
m=F_{j_{1}}+\cdots+F_{j_{s(m)}},
$$

with $1 \leq j_{s(m)}<\cdots<j_{1}$. Moreover, this decomposition is unique.

Proof: If $m$ is a Fibonacci number the proof is ended. Although, we have that $F_{j_{1}}<$ $m<F_{j_{1}+1}$, and so, $0<m-F_{j_{1}}<F_{j_{1}-1}$. Then, it implies that $F_{j_{2}} \leq m-F_{j_{1}}<F_{j_{1}-1}$, with $j_{2}<j_{1}-1$. Now, if $m-F_{j_{1}} \neq F_{j_{2}}$ we only have to reiterate the same process.

At this point we can give what is the basic idea to prove Lemma 8: if we look at the Diophantine condition (4) for $\omega=2 \pi \gamma$, which is verified with $\sigma=2$, the estimate that we obtains for $\max _{n \in \mathbb{Z}}\left\{|m \gamma-n|^{-1}\right\}$ is of order $\mathcal{O}(m)$. But we are going to see that, in fact, it is $\mathcal{O}\left(F_{j_{s(m)}}\right)$, where $F_{j_{s(m)}}$ is the smaller Fibonacci number in the decomposition of $m$ provided by Lemma 9 . In the case when $m$ is a Fibonacci number $F_{k}$, the value of the "small divisor" $\max _{n \in \mathbb{Z}}\left\{|m \gamma-n|^{-1}\right\}$ can be easily computed. It is obtained by taking $n=F_{k-1}$ :

$$
F_{k} \gamma-F_{k-1}=(-1)^{k} \gamma^{k+1}
$$

For the general case, we have the following result.

Lemma 10 Let $m \in \mathbb{N}$. Then, for any $n \in \mathbb{Z}$ we have:

$$
|m \gamma-n| \geq \gamma^{j_{s(m)}+1}\left(\frac{1-2 \gamma^{2}}{1-\gamma^{2}}\right)
$$

where $j_{s(m)}$ is provided by Lemma 9 .

Proof: We consider the decomposition of $m$ in Fibonacci numbers given by Lemma 9 . Then, we have

$$
\begin{aligned}
|m \gamma-n| \geq & \left|F_{j_{s(m)}} \gamma-\left(n-F_{j_{1}}-\cdots-F_{j_{s(m)-1}}\right)\right| \\
& -\left|F_{j_{1}} \gamma-F_{j_{1}-1}\right|-\cdots-\left|F_{j_{s(m)-1}} \gamma-F_{j_{s(m)-1}-1}\right| \\
\geq & \gamma^{j_{s(m)}+1}-\gamma^{j_{1}+1}-\cdots-\gamma^{j_{s(m)-1}+1} \geq \gamma^{j_{s(m)}+1}-\frac{\gamma^{j_{s(m)-1}+1}}{1-\gamma^{2}} \\
\geq & \gamma^{j_{s(m)}+1}\left(1-\frac{\gamma^{2}}{1-\gamma^{2}}\right)=\gamma^{j_{s(m)}+1}\left(\frac{1-2 \gamma^{2}}{1-\gamma^{2}}\right)
\end{aligned}
$$


where we have strongly used that the decomposition of Lemma 9 does not contain consecutive Fibonacci numbers.

As consequence of this result, it is clear that now the key point is to bound the cardinal of the following sets,

$$
R(j, k)=\#\left\{m \in \mathbb{N}: 1 \leq m<F_{k}, j_{s(m)}=j\right\} .
$$

Lemma 11 For any $k \geq 1$ and for any $j \leq k$, we have that $R(j, k+1) \leq F_{k-j}$.

Proof: Let us consider $1 \leq m<F_{k+1}$ such that $j_{s(m)}=j$. If we consider the decomposition of $m$ in Fibonacci numbers of Lemma 9, then we have that $m=F_{j_{s(m)}}$, or although, we have the following possibilities for $j_{s(m)-1}: j_{s(m)-1}=j_{s(m)}+2, \ldots, k$. Then, we can give the following (pessimistic) estimate for $R(j, k+1)$ :

$$
R(j, k+1) \leq 1+R(j+2, k+1)+R(j+3, k+1)+\cdots+R(k, k+1) .
$$

Moreover, it is clear that $R(k, k+1)=1$ and $R(k-1, k+1)=1$. Now, in order to obtain a more explicit estimate for $R(j, k+1)$, we define a new sequence of numbers $\mathcal{R}(j, k+1)$ by means of the equality:

$$
\mathcal{R}(j, k+1)=1+\mathcal{R}(j+2, k+1)+\cdots+\mathcal{R}(k, k+1),
$$

with $\mathcal{R}(k, k+1)=\mathcal{R}(k-1, k+1)=1$. From this definition, it is clear that $R(j, k+1) \leq$ $\mathcal{R}(j, k+1)$. Moreover, we have

$$
\mathcal{R}(j, k+1)=\mathcal{R}(j+1, k+1)+\mathcal{R}(j+2, k+1),
$$

and so, $\mathcal{R}(\cdot, k+1)$ verifies the Fibonacci recurrence, but with respect to a decreasing index. This implies that $\mathcal{R}(j, k+1)=F_{k-j}$.

From here, it is not difficult to obtain the following result, that it is basically equivalent to the desired bound:

Lemma 12 There exists a constant $K_{1}>0$, such that for any $k \geq 1$, we have

$$
\sum_{m=1}^{F_{k+1}-1} \max _{n \in \mathbb{Z}}\left\{|m \gamma-n|^{-1}\right\} \leq K_{1} k F_{k+1} .
$$

Proof: We only have to apply the results of Lemma 10 and Lemma 11.

$$
\begin{aligned}
\sum_{m=1}^{F_{k+1}-1} \max _{n \in \mathbb{Z}}\left\{|m \gamma-n|^{-1}\right\} & \leq \sum_{j=1}^{k} R(j, k+1) \gamma^{-j-1}\left(\frac{1-\gamma^{2}}{1-2 \gamma^{2}}\right) \\
& =\left(\frac{1-\gamma^{2}}{1-2 \gamma^{2}}\right) A\left(\frac{1}{\gamma}\right)^{k+1} \sum_{j=1}^{k}\left(1+(-1)^{k-j} \frac{B}{A} \gamma^{2 k-2 j}\right) \\
& \leq K_{2}\left(\frac{1}{\gamma}\right)^{k}\left(k+K_{3}\right),
\end{aligned}
$$

where $K_{2}$ and $K_{3}$ are constants independent of $k$. From here the result follows.

Now, the proof of Lemma 8 is clear. We only have to apply Lemma 12 with $k$ taken in such a way that $F_{k} \leq M<F_{k+1}$, and to use that $k \leq K_{4} \log (M+1)$, for certain $K_{4}$ independent of $M$ (look at the explicit expression (112) for $F_{k}$ ). 


\section{Acknowledgements}

The authors want to thank $\mathrm{R}$. de la Llave for interesting discussions and remarks. This work has been partially supported by the grants NATO CRG950273, the Catalan grant CIRIT SRC-98-0042 and the INTAS project 97-10771. T.M. Seara is also supported by the Spanish grant DGICYT PB94-0215.

\section{References}

[1] D.K. Arrowsmith, C.M. Place, An Introduction to Dynamical Systems, Cambridge University Press, 1990.

[2] A. Berretti, A. Celletti, L. Chierchia, C. Falcolini, "Natural Boundaries for AreaPreserving Twist Maps", J. Stat. Phys. 66 (1992), pp. 1613-1630.

[3] A. Berretti, L. Chierchia, "On the Complex Analytic Structure of the Golden Invariant Curve for the Standard Map", Nonlinearity 3 (1990), pp. 39-44.

[4] B.V. Chirikov, "A Universal Instability of Many-Dimensional Oscillator Systems", Physics Reports 52 (1979), pp. 263-279.

[5] A.M. Davie, "The Critical Function for the Semistandard Map", Nonlinearity 7, (1994) pp. 219-229.

[6] J.M. Greene, "A Method for Determining a Stochastic Transition", J. Math. Phys. 20 (1979), pp. 1183-1201.

[7] J.M. Greene, I.C. Percival, "Hamiltonian Maps in the Complex Plane", Physica 3D (1981), pp. 530-548.

[8] V.F. Lazutkin, "Splitting of Separatrices for the Chirikov's Standard Map", VINITI 6372/84. Preprint (1984).

[9] R. de la Llave, D. Rana, "Accurate Strategies for K.A.M. bounds and their Implementation", Computer Aided Proofs in Analysis, K. Meyer and D. Schmidt (Eds.), IMA Vol. Math. Appl. 28, Springer, New York (1991), pp. 127-146.

[10] R.S. MacKay, Renormalization in Area Preserving Maps, Ph. D. Thesis, Princeton University, 1982. World Scientific, London, 1993.

[11] R.S. MacKay, Transition to Chaos for Area-preserving Maps, Springer Lect. Notes in Physics, vol. 247 (1985), p. 390.

[12] C.L. Siegel, J.K. Moser, Lectures on Celestial Mechanics, Springer-Verlang, Berlin, 1971.

[13] C. Simó, "Averaging Under Fast Quasiperiodic Forcing", in J. Seimenis (Ed.), Hamiltonian Mechanics, Integrability and Chaotic Behaviour, NATO Adv. Sci., Inst. Ser. B Phys., vol. 331, Plenum Press, New York, 1994, pp. 13-34. 